\author{
Aus der Abteilung \\ Psychosomatische Medizin und Psychotherapie \\ (Prof. Dr. med. C. Herrmann-Lingen) \\ des Zentrums Psychosoziale Medizin \\ der \\ Medizinischen Fakultät der Universität Göttingen
}

\title{
Bestimmung \\ Zentraler Beziehungskonfliktthemen \\ (nach Luborsky) \\ bei Patienten mit generalisierter Angststörung
}

Vergleich der Qualität unterschiedlicher Auswertungsmethoden und Therapieverfahren

\author{
INAUGURAL-DISSERTATION \\ zur Erlangung des Doktorgrades \\ der Medizinischen Fakultät \\ der Georg-August-Universität zu Göttingen
}

vorgelegt von
Christiane Oelze
aus
Bühl/Baden

Göttingen 2012 
Dekan:

I. Berichterstatter:

II. Berichterstatter/in:

III. Berichterstatter/in:
Prof. Dr. rer. nat. H.K. Kroemer

Prof. Dr. med. H. Staats

Priv.-Doz. Dr. med. Wedekind

Prof. Dr. rer. nat. Virsik-Köpp

Tag der mündlichen Prüfung: 13.05.2013 


\section{Inhaltsverzeichnis}

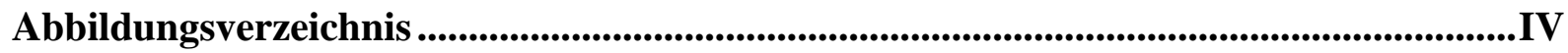

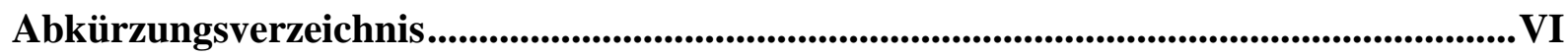

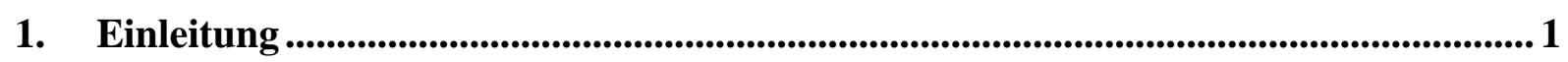

1.1. Die Methode des zentralen Beziehungskonfliktthemas - ZBKT ................................. 1

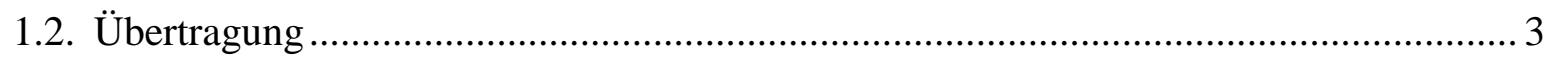

1.3. Die generalisierte Angststörung - Entstehungsmodell und Empirie............................. 4

1.4. Die generalisierte Angststörung - Diagnosestellung und Therapie .............................5

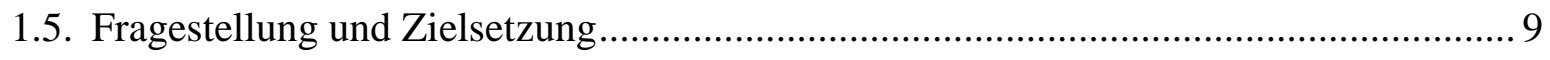

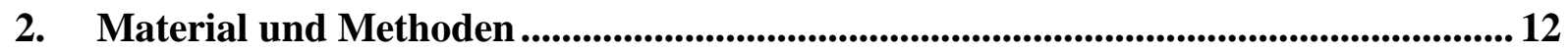

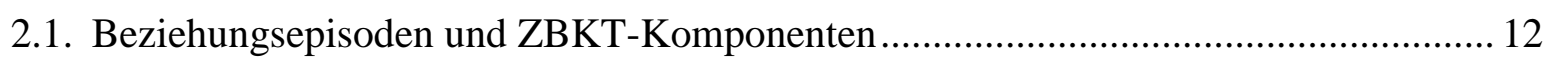

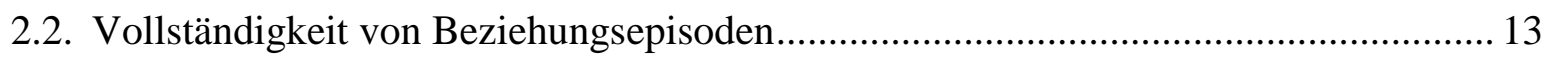

2.3. Bestimmung von Hauptperson und Zeitebene ...................................................... 13

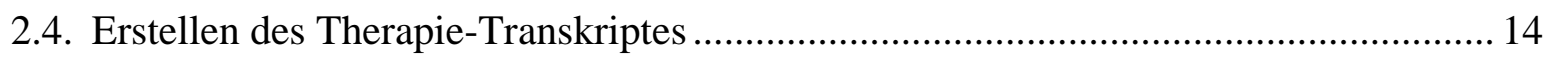

2.5. Zuordnung zu Standardkategorien und Clustern ....................................................... 14

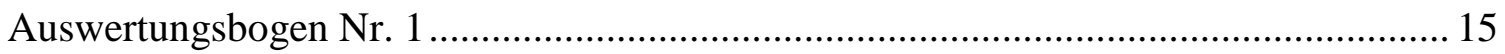

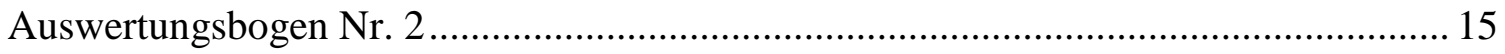

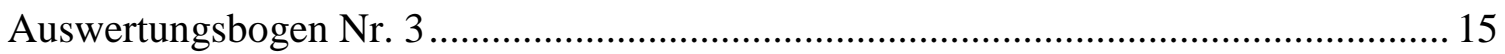

2.6. ZBKT im Auszählverfahren vs Tailormade-ZBKT …................................................ 16

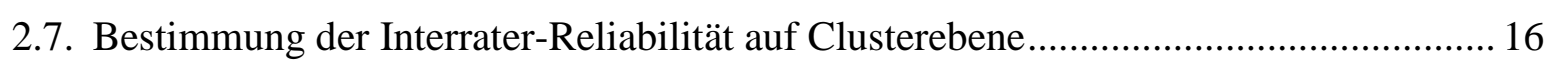

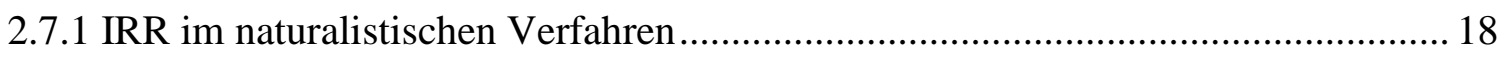

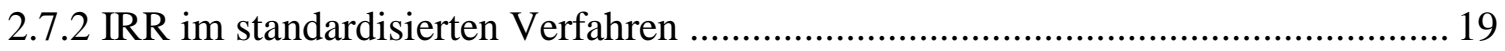

2.8. Patientenkollektiv, Aufnahme- und Ausschlusskriterien ......................................... 19

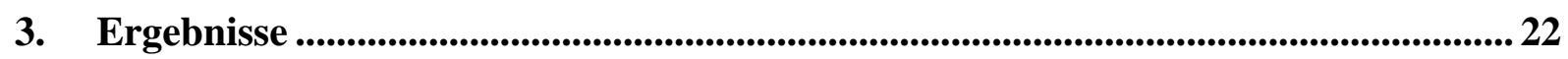

3.1. Die Interrater-Reliabilität für das ZBKT im Auszählverfahren .................................. 22

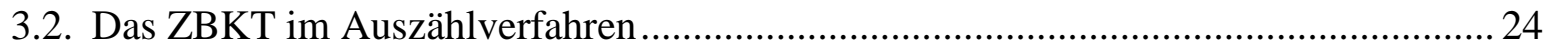

3.2.1 Klassische ZBKT-Cluster - die häufigsten W-, RO- und RS-Kategorien ............... 24

3.2.2 Das AV-ZBKT (klassische ZBKT-Cluster) des Gesamtkollektivs........................ 25

3.2.3 LU-Cluster - die häufigsten WO-, WS-, RO- und RS-Kategorien ......................... 26

3.2.4 Das AV-ZBKT (LU-Cluster) des Gesamtkollektivs ............................................ 28

3.3. Visualisierung - Erkennen häufigster Wünsche und Reaktionen im Gesamtkollektiv 29

3.4. Die Häufigkeitsverteilung der klassischen ZBKT-Cluster bei SET- und VT-

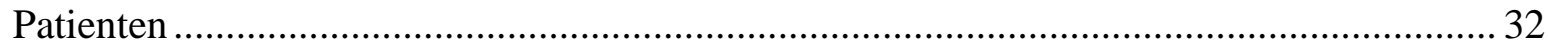

3.4.1 Die häufigsten W-, RO- und RS-Kategorien der SET- und VT-Patienten .............. 32 


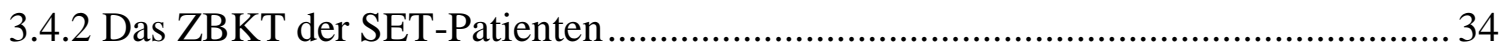

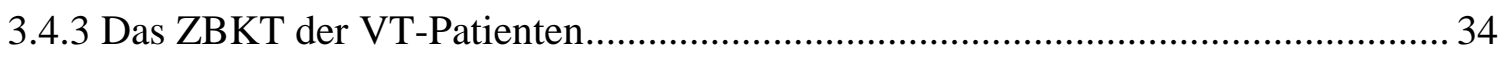

3.5. Die Häufigkeitsverteilung der LU-Cluster bei SET- und VT-Patienten ...................... 35

3.5.1 Die häufigsten W-, RO- und RS-Kategorien der SET- und VT-Patienten .............. 35

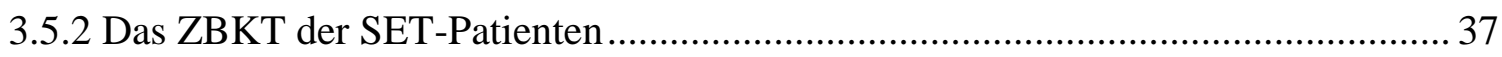

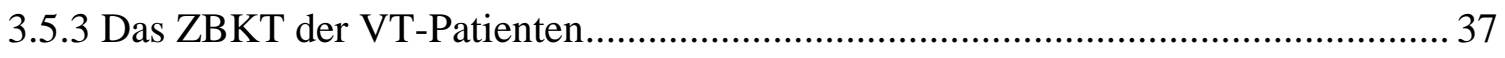

3.6. Die Veränderungen der Häufigkeitsverteilung klassischer ZBKT-Cluster im

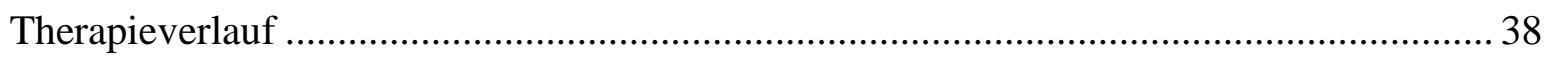

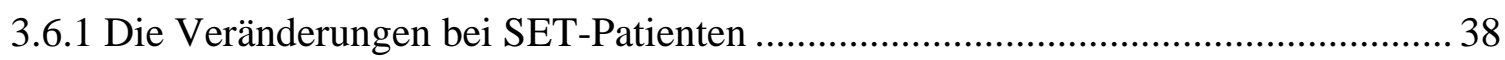

3.6.2 Das ZBKT der SET-Patienten zu verschiedenen Zeitpunkten (T1/T2) ................. 40

3.6.3 Die Veränderungen bei VT-Patienten ............................................................ 41

3.6.4 Das ZBKT der VT-Patienten zu verschiedenen Zeitpunkten (T1/T2) ................. 44

3.7. Die Veränderungen der Häufigkeitsverteilung der LU-Cluster im Therapieverlauf.... 45

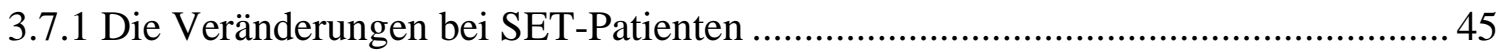

3.7.2 Das LU-ZBKT der SET-Patienten zu verschiedenen Zeitpunkten (T1/T2)............ 49

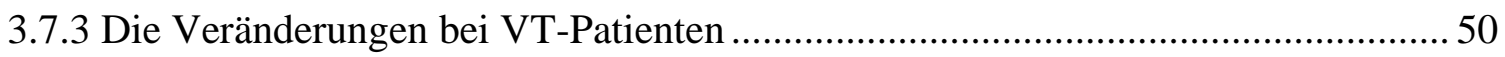

3.7.4 Das LU-ZBKT der VT-Patienten zu verschiedenen Zeitpunkten (T1/T2) ............. 54

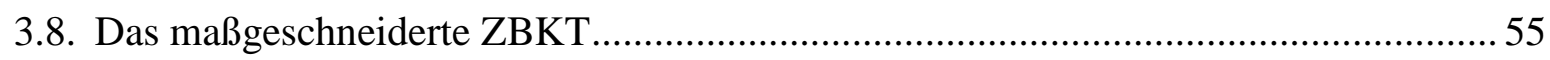

3.8.1 TM-ZBKT der SET-Patienten (T1/T2) ............................................................. 55

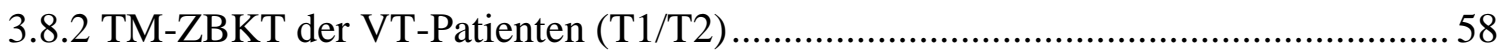

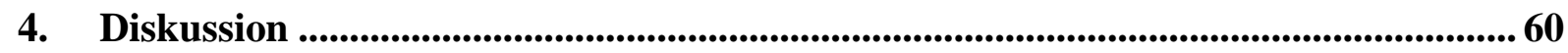

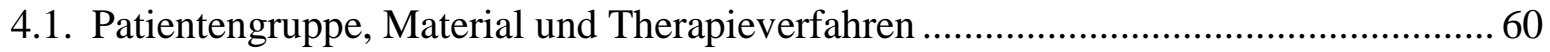

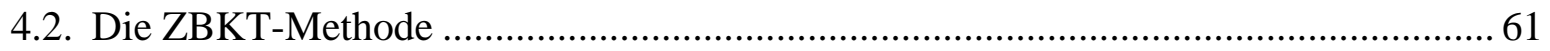

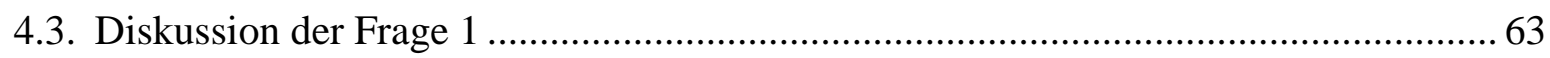

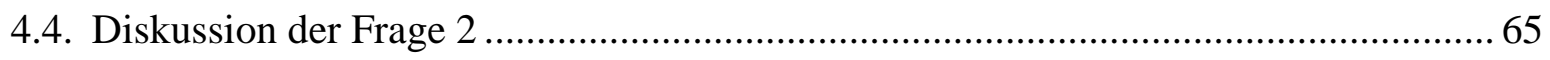

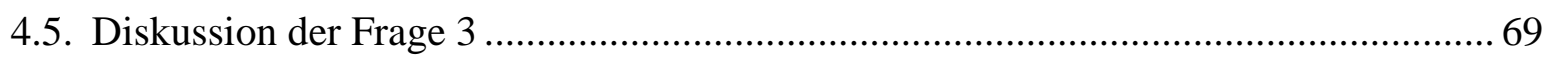

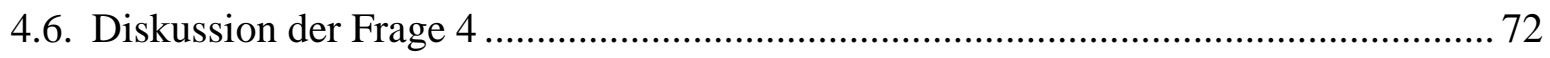

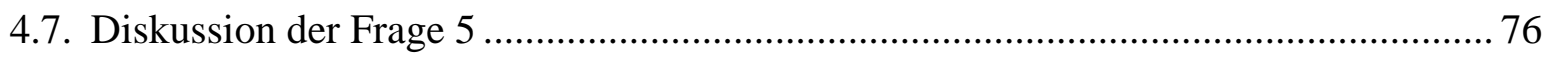

5. Zusammenfassung ...................................................................................................................... 78

6. Anhang (Auswertungsbogen und Clusterkategorien) ...................................................8 81

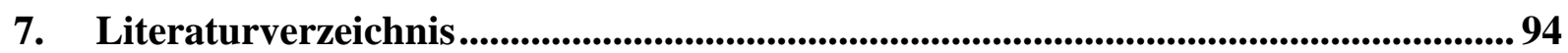




\section{Abbildungsverzeichnis}

Abb. 1 Veränderungen der GAS-Diagnose im DSM-Klassifikationssystem ........................... 6

Abb. 2 Bewertung der Vollständigkeit von Beziehungsepisoden ........................................ 13

Abb. 3 Beispielrechnung Interrater-Reliabilität .............................................................. 17

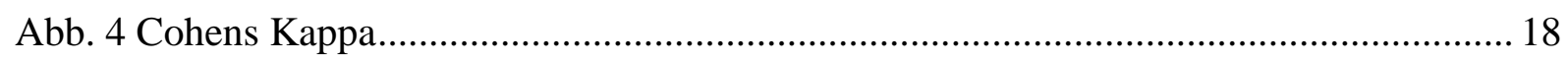

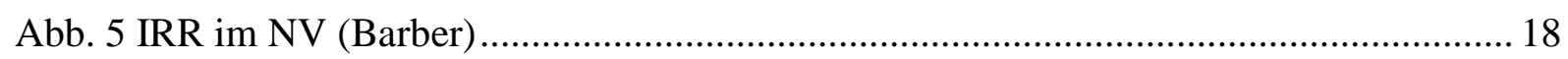

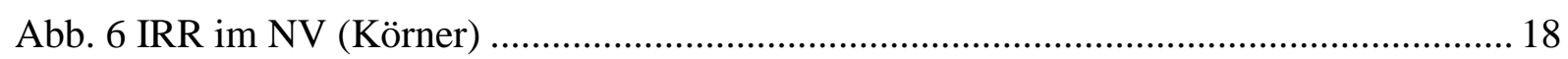

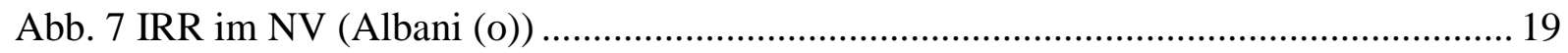

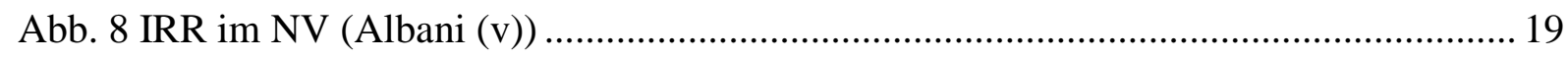

Abb. 9 Allgemeine Angaben zum Patientenkollektiv ...................................................... 20

Abb. 10 Ausbildungsstand des Patientenkollektives........................................................... 20

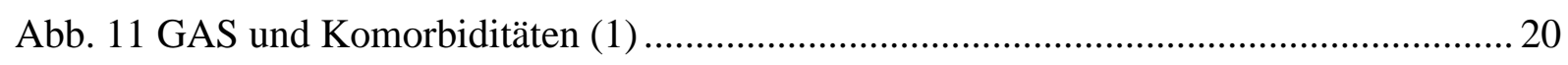

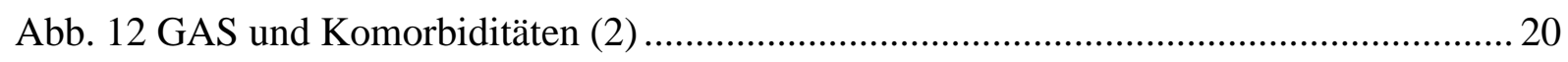

Abb. 13 Aufnahme- und Ausschlusskriterien der Studie ...................................................... 21

Abb. 14 Materialverlust durch akustischen Mangel der Tonbandaufnahme........................... 21

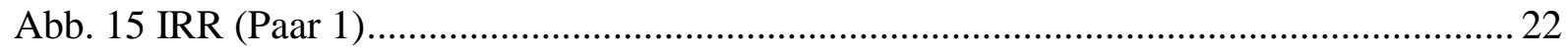

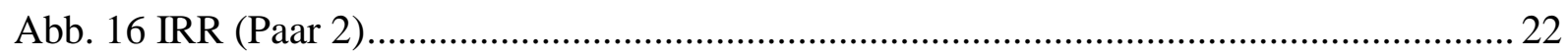

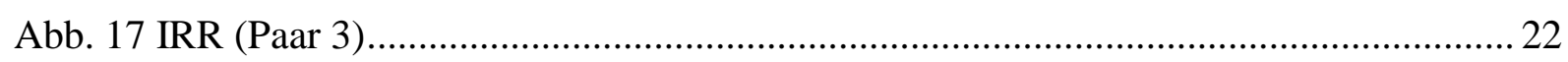

Abb. 18 Häufigkeit der klassischen ZBKT-W-Cluster im Gesamtkollektiv ........................... 24

Abb. 19 Häufigkeit der klassischen ZBKT-RO-Cluster im Gesamtkollektiv .......................... 25

Abb. 20 Häufigkeit der klassischen ZBKT-RS-Cluster im Gesamtkollektiv ......................... 25

Abb. 21 Häufigkeit der LU-WO-Cluster im Gesamtkollektiv ............................................... 26

Abb. 22 Häufigkeit der LU-WS-Cluster im Gesamtkollektiv................................................. 27

Abb. 23 Häufigkeit der LU-RO-Cluster im Gesamtkollektiv ................................................ 27

Abb. 24 Häufigkeit der LU-RS-Cluster im Gesamtkollektiv.................................................. 28

Abb. 25 Visualisierung der häufigsten W im Gesamtkollektiv (klassische W-Cluster).......... 29

Abb. 26 Visualisierung der häufigsten W im Gesamtkollektiv (LU-WO-Cluster).................. 29

Abb. 27 Visualisierung der häufigsten W im Gesamtkollektiv (LU-WS-Cluster) ................... 29

Abb. 28 Visualisierung der häufigsten RO im Gesamtkollektiv (klassische Cluster) ............. 30

Abb. 29 Visualisierung der häufigsten RO im Gesamtkollektiv (LU-Cluster) ........................ 30

Abb. 30 Visualisierung der häufigsten RS im Gesamtkollektiv (klassische Cluster) .............. 31

Abb. 31 Visualisierung der häufigsten RS im Gesamtkollektiv (LU-Cluster) ....................... 31

Abb. 32 Häufigkeit der klassischen ZBKT-W-Cluster bei SET- und VT-Patienten ............... 32

Abb. 33 Häufigkeit der klassischen ZBKT-RO-Cluster bei SET- und VT-Patienten .............. 33

Abb. 34 Häufigkeit der klassischen ZBKT-RS-Cluster bei SET- und VT-Patienten ............... 33

Abb. 35 Häufigkeit der LU-WO-Cluster bei SET- und VT-Patienten.................................... 35 
Abb. 36 Häufigkeit der LU-WS-Cluster bei SET- und VT-Patienten 36

Abb. 37 Häufigkeit der LU-RO-Cluster bei SET- und VT-Patienten 36

Abb. 38 Häufigkeit der LU-RS-Cluster bei SET- und VT-Patienten 37

Abb. 39 Veränderung der Häufigkeit klassischer ZBKT-W-Cluster von SET-Patienten........ 38

Abb. 40 Visualisierung der Veränderung des Wunsch-Clusters............................................. 38

Abb. 41 Veränderung der Häufigkeit klassischer ZBKT-RO-Cluster von SET-Patienten...... 39

Abb. 42 Veränderung der Häufigkeit klassischer ZBKT-RS-Cluster von SET-Patienten ...... 40

Abb. 43 Visualisierung der Veränderung des RS-Clusters ................................................... 40

Abb. 44 Veränderung der Häufigkeit klassischer ZBKT-W-Cluster von VT-Patienten ......... 41

Abb. 45 Visualisierung der Veränderung des W-Clusters ..................................................... 41

Abb. 46 Veränderung der Häufigkeit klassischer ZBKT-RO-Cluster von VT-Patienten........ 42

Abb. 47 Veränderung der Häufigkeit klassischer ZBKT-RS-Cluster von VT-Patienten ........ 43

Abb. 48 Visualisierung der Veränderung des RS-Clusters .................................................... 43

Abb. 49 Veränderung der Häufigkeit des LU-WO-Clusters von SET-Patienten ..................... 45

Abb. 50 Visualisierung der Veränderung des WO-Clusters .................................................. 45

Abb. 51 Veränderung der Häufigkeit des LU-WS-Clusters von SET-Patienten ..................... 46

Abb. 52 Visualisierung der Veränderung des WS-Clusters ..................................................... 46

Abb. 53 Veränderung der Häufigkeit des LU-RO-Clusters von SET-Patienten....................... 47

Abb. 54 Veränderung der Häufigkeit des LU-RS-Clusters von SET-Patienten ...................... 48

Abb. 55 Visualisierung der Veränderung des RS-Clusters ..................................................... 48

Abb. 56 Veränderung der Häufigkeit des LU-WO-Clusters von VT-Patienten ....................... 50

Abb. 57 Visualisierung der Veränderung des WO-Clusters .................................................... 50

Abb. 58 Veränderung der Häufigkeit des LU-WS-Clusters von VT-Patienten ....................... 51

Abb. 59 Visualisierung der Veränderung des WS-Clusters ................................................... 51

Abb. 60 Veränderung der Häufigkeit des LU-RO-Clusters von VT-Patienten ........................52

Abb. 61 Veränderung der Häufigkeit des LU-RS-Clusters von VT-Patienten ......................... 53

Abb. 62 Visualisierung der Veränderung des RS-Clusters bei VT-Patienten........................... 53 


\section{Abkürzungsverzeichnis}

aCL alternative Cluster

APA American Psychiatric Association

AV Auszählverfahren

BE Beziehungsepisode(n)

CCRT Core Conflictual Relationship Theme

CL Clusterkategorie(n)

DFG Deutsche Forschungsgemeinschaft

DIMDI Deutsches Institut für medizinische Dokumentation und Information

DSM Diagnostisches und statistisches Manual psychischer Störungen

GAS Generalisierte Angststörung

ICD International Classification of Deseases

IR Interrater

IRR Interrater-Reliabilität

K Kappa

LU ZBKT-Arbeitsgruppe Leipzig/Ulm, Modifikation der ZBKT-Methode "logically unified"

LU (o) LU-Cluster: originale Version mit Subkategorien

LU (v) LU-Cluster: vereinfachte Version ohne Subkategorien

m männlich

NRO Negative Reaktion des Objektes

NRS Negative Reaktion des Subjektes

NV Naturalistisches Verfahren

PRO Positive Reaktion des Objektes

PRS Positive Reaktion des Subjektes

RAP Relationship Anecdote Paradigm

RO Reaktion des Objektes

RRMC Rollen-Beziehungmodell-Konfiguration

RS Reaktion des Subjektes

rSK Reformulierte Standardkategorie(n)

SASB Strukturale Analyse sozialen Verhaltens

SET Supportiv Expressive Therapie

SK Standardkategorie(n)

SKID Strukturierte klinische Interviews für DSM-IV

SV Standardisiertes Verfahren

TM Tailormade (deutsch: maßgeschneidert) 
TM-ZBKT Maßgeschneidertes Zentrales Beziehungskonfliktthema

u.a. unter anderem

vS versus

VT Verhaltenstherapie

W weiblich

$\mathrm{W}$

Wunsch

WO Wunsch an Objekt

WS Wunsch an Subjekt

ZBKT Zentrales Beziehungskonfliktthema 


\section{Einleitung}

Lester Luborsky (1977, 1998a) etablierte ein Verfahren zur Bestimmung des „Zentralen Beziehungs-Konfliktthemas“ (ZBKT), welches die Arbeitsgrundlage der vorliegenden Dissertation bildet. Das Verfahren ermöglicht es, das Phänomen der Übertragung (Freud 1912/2006) empirisch zu messen (Luborsky et al. 1985). Die Messungen werden an bestimmten Patientenaussagen vorgenommen, welche in mehreren Arbeitsschritten aus Psychotherapiestunden gefiltert werden. Dem Aspekt der Häufigkeit, mit der diese Aussagen fallen und sich wiederholen, gilt besondere Aufmerksamkeit, da Häufigkeit in gewisser Weise für Wichtigkeit steht (Murray 1938), sich durchsetzt und somit zur Bestimmung von Übertragung herangezogen werden kann. Mit der ZBKT-Methode werden die häufigsten Patientenaussagen erfasst und in einem längeren Prozess schließlich das Zentrale Beziehungskonfliktthema aus ihnen ermittelt. Das Verfahren wird heute an einer breitgefächerten Patientengruppe angewendet und gehört zu den am häufigsten eingesetzten Methoden zur Untersuchung von Beziehungsmustern in psychodynamischen Therapieverfahren (Luborsky et al. 1999).

Die Autorin und zwei weitere medizinische Doktoranden untersuchten Psychotherapiestunden von 45 Patienten mit der Hauptdiagnose generalisierte Angststörung (GAS). Zuvor wurden sie unter der Leitung von Herrn Prof. Dr. med. H. Staats geschult, um die Methode zur Bestimmung des ZBKT`s nach den Vorgaben des Manuals zur ZBKT-Methode (Luborsky et al. 1991) zu erlernen. Die untersuchten Patienten nahmen an einer randomisierten, kontrollierten klinischen Studie für Kurzzeittherapie teil, welche in der Abteilung für Psychosomatik und Psychotherapie der Universität Göttingen von Mai 2001 bis Juni 2007 durchgeführt wurde.

Während 25 der insgesamt 45 untersuchten Patienten verhaltenstherapeutisch behandelt wurden, erhielten 20 Patienten eine auf GAS zugeschnittene psychoanalytisch orientierte Fokaltherapie (SET) (Leichsenring et al. 2005). Das Erheben und Vergleichen von ZBKT`s unterschiedlicher Behandlungsgruppen stellt einen zentralen Teil der vorliegenden Dissertation dar, mit welchem wir auf eine der wichtigsten Forderungen aktueller und zukünftiger ZBKT-Forschung eingehen (Albani et al. 2008). Vergleiche psychodynamischer und verhaltenstherapeutischer Therapieverfahren zur Behandlung von GAS wurden von Leichsenring et al. (2009), Wiegand (2010) und Werling (2010) vorgelegt. Auch mit der vorliegenden Arbeit wird ein Beitrag zur Untersuchung psychodynamischer Therapieverfahren bei GAS-Patienten geleistet.

\subsection{Die Methode des zentralen Beziehungskonfliktthemas - ZBKT}

Lester Luborsky entwickelte ab 1977 das Verfahren zur Bestimmung des ZBKT (englisch: CCRT = Core Conflictual Relationship Theme), welches genutzt wird, um Übertragungsmuster zu operationalisieren. Dazu werden klassischerweise bestimmte Textteile verwendet, die in Psychotherapiestunden regelmäßig vorkommen und Interaktionen beinhalten. Diese sogenannten Narrative weisen typische Erkennungsmerkmale auf. Während Patienten Begegnungen mit anderen Menschen schildern, tauchen darin implizit und explizit 
geäußerte Wünsche (W) auf, welche sie an ihr Gegenüber richten. Es folgen Reaktionen des Gegenübers auf diese Wünsche (Reaktion des Objekts, RO) und schließlich die Reaktionen des Patienten auf die Reaktionen des Gegenübers (Reaktion des Subjekts, RS). Über das Erfassen der häufigsten Wünsche und Reaktionen wird das Benennen eines übergeordneten Beziehungsmusters operationalisiert. ,Übertragung ist ein ubiquitäres Phänomen, das in allen menschlichen Beziehungen eine mehr oder weniger große Rolle spielt" (König 1998, S.12). Das übergeordnete Muster findet sich also auch in der Beziehung zum Therapeuten und zeigt einen auf viele Situationen übertragenen zentralen Beziehungskonflikt.

Sigmund Freud nahm an, dass Heilung durch die Analyse von Übertragung zustande kommt (vgl. Luborsky und Crits-Christoph 1998, IX). König (1998) beschreibt, wie man sich Übertragung in psychoanalytischen Therapieverfahren zunutze macht, um Veränderungen zu erreichen. Das einst von Freud (1912/2006) benannte Konzept der Übertragung wird durch die ZBKT-Methode neu beschrieben, weshalb sie als Fremdbeurteilungsinstrument, das Übertragung zu operationalisieren und somit zu erfassen vermag, verstanden werden kann. Das ZBKT als ein Maß für Übertragung zu bezeichnen, sei gerechtfertigt, weil es Freuds Beobachtungen zur Übertragung entspricht (Luborsky et al. 1985). Luborsky (z.B. 1998 b) trug die Übereinstimmungen zwischen Freuds Übertragungsbeobachtungen und den Ergebnissen der ZBKT-Methode zusammen.

Um die zentralen Themen verschiedener Patienten miteinander vergleichen $\mathrm{zu}$ können, wurden übergeordnete Standardkategorien (SK) (Crits-Christoph und Demorest 1988) entwickelt, denen die ZBKT-Komponenten W, RO und RS inhaltlich zugeordnet werden. Es stehen 34 Kategorien für Wünsche und jeweils 30 Kategorien für Reaktionen der Objekte und Subjekte zur Verfügung. Für die W- RO- und RS-Komponente wurden jeweils 8 zugehörige Cluster (CL) entwickelt (Barber et al. 1990).

Die ursprünglichen SK gerieten wiederholt in die Kritik. Problematisiert wurde zum Beispiel die unterschiedliche inhaltliche Nähe der Kategorien, welche die eindeutige Zuordnung der ZBKT-Komponenten erschwert. (z.B. SK für die W-Komponente: 1: Verstanden werden, 2: angenommen akzeptiert werden, 3: respektiert werden). Hierdurch kann sich eine Varianz für die Nennungshäufigkeit einer Kategorie entwickeln. Auch das Fehlen von Kategorien für klinisch bedeutsame Inhalte wird als Schwierigkeit der ursprünglichen SK gewertet. Weiterhin ist eine gewisse Subjektivität bei der Zuordnung zu den SK und CL nicht auszuschließen.

Verbesserungsversuche für die Clustersysteme wurden von Crits-Christoph und Baranackie (1992/2004), aber auch von Albani et al. (1999) vorgelegt. Eine umfassende inhaltliche Revision des Kategoriensystems setzten Albani et al. (2002 a,b) schließlich als reformulierte Standardkategorien (rSK) um. Da die ursprünglichen CL der SK von Barber und Luborsky ungeprüft aus der amerikanischen Version übersetzt und übernommen worden waren, entwickelten Körner et al. (2002) alternative Cluster (aCL) auf der Grundlage der bestehenden CL nach Barber et al. (1990). Die interne Konsistenz der aCL sei für den deutschsprachigen Raum größer als die der ursprünglichen, angloamerikanischen Version (Körner et al. 2002).

Während Barber et al. (1990) und Körner et al. (2002) für jede ZBKT-Komponente (W, RO, RS) separate SK und CL bereit halten, bieten Albani et al. (2002 a,b) einen gemeinsamen Pool an SK, denen die ZBKT-Komponenten inhaltlich zugeordnet werden. Die rSK lassen sich in harmonische (A-D) und disharmonische (E-M) Sequenzen unterteilen und sind in sich hierarchisch gegliedert. Jedem Buchstaben ist eine Qualität zugeordnet (z.B. A ,sich zuwenden", A1 explodieren, bewundern, A1.1 neugierig sein, interessieren, erforschen, offensein). Innerhalb der hierarchischen Gliederung einer Qualität kann die Zuteilung fein 
abgestimmt werden. Zudem weisen die rSK eine sogenannte „vierte Dimension“ auf, eine Differenzierung der Wunschkomponente in Wünsche an andere (WO) und Wünsche des Subjekts an bzw. für sich selbst (WS). Hierdurch soll eine Steigerung der InterraterReliabilität (IRR) erreicht werden. Bei der Entwicklung der rSK lagen Albani et al. (2002 a,b) weit mehr empirische Daten zugrunde, was zu einer höheren internen Konsistenz beiträgt.

Alle Methoden etablieren über die Zuordnung zu SK und CL eine Abstraktionsebene, auf der W, RO und RS von der ursprünglichen Formulierung gelöst dargestellt werden können, ohne jedoch viel an Interpretation einfließen $\mathrm{zu}$ lassen. Somit lassen sich individuelle Formulierungen von Patienten miteinander vergleichen.

In psychodynamischen Therapieverfahren wird die ZBKT-Methode heute zur Behandlung definierter Krankheitsbilder (Crits-Christoph et al. 1995) und für Prozessuntersuchungen in Einzeltherapien (Luborsky et al. 1999) eingesetzt. Außerdem findet die ZBKT-Methode zur Erfassung von repetitiven Beziehungsmustern auch bei Kindern (Luborsky et al. 1998), Männern und Frauen (Cierpka et al. 1992/2001; Staats 1996), depressiven (Vanheule et al. 2006) und persönlichkeitsgestörten Patienten (Drapeau und Perry 2004) Anwendung. Die Methode zeigt eine mehrfach nachgewiesene gute IRR und gehört $\mathrm{zu}$ den am häufigsten eingesetzten Methoden zur Untersuchung von Beziehungsmustern in psychodynamischen Psychotherapien (Luborsky et al. 1999).

Verglichen mit anderen Fremdbeurteilungsinstrumenten zur Operationalisierung von Übertragung, z.B. der „Rollen-Beziehungsmodell-Konfiguration“ (RRMC) nach Horowitz (1991), oder der „strukturale(n) Analyse sozialen Verhaltens“ (SASB) (Benjamin 1993; Tress et al. 1990) ist die ZBKT-Methode relativ gesehen weniger zeitaufwendig, wodurch sie sich auch zur Untersuchung größerer Fallzahlen eignet.

\section{2. Übertragung}

Die Bedeutung des Übertragungsbegriffes wurde bereits vielfach umschrieben. Greenson (1967/1986, S. 163) bezeichnet sie als

„...eine besondere Art der Beziehung zu einer Person; sie ist ein besonderer Typus von Objektbeziehung. Das Hauptmerkmal ist das Erleben von Gefühlen einer Person gegenüber, die zu dieser Person gar nicht passen und die sich in Wirklichkeit auf eine andere Person beziehen. Im Wesentlichen wird auf eine Person in der Gegenwart so reagiert, als sei sie eine Person in der Vergangenheit. Übertragung ist eine Wiederholung, eine Neuauflage einer alten Objektbeziehung. Sie ist ein Anachronismus, ein Irrtum in der Zeit. Eine Verschiebung hat stattgefunden; Triebimpulse, Gefühle und Abwehrhaltungen, die sich auf eine Person in der Vergangenheit beziehen, sind auf eine Person in der Gegenwart verschoben worden."

Übertragung ist ein Phänomen, welches viele beliebige Situationen, in der Menschen interagieren, beeinflusst. Beziehungen früherer Zeit können in gegenwärtigen Beziehungen partiell wiedererlebt werden. Konflikte können in der neuen Beziehung erneut ausgetragen werden (König 1998). Jede Person handelt aufgrund ihrer persönlichen Erfahrungen im Umgang mit anderen Menschen überwiegend unbewusst nach einem bestimmten Muster und überträgt deshalb Erwartungen, Wünsche oder auch Befürchtungen auf ihr aktuelles 
Gegenüber. Bestimmte Beziehungsmuster werden immer wieder abgerufen und wie eine Schablone auf alltägliche, sozial interaktive Begegnungen gelegt. Übertragungsmuster können durch gewisse Eigenschaften des Gegenübers aktiviert werden, weshalb man sie „Übertragungsauslöser“ nennt (z.B. Greenson 1967/1986, König 1998). In Therapiestunden können Idiosynkrasien des Therapeuten (Greenson 1967/1986) „Übertragungsauslöser“ sein.

Sigmund Freud (1912/2006, S. 41) prägte den Begriff der Übertragung, empfand sie zunächst aber als störendes Element, bevor er ihren Nutzen für die therapeutische Arbeit erkennen konnte. Er beschrieb sie als „stärksten Widerstand“ und „methodischen Nachteil“", der der Behandlung während der Analyse entgegentreten könne.

Das Erfassen und Analysieren von Übertragungsaspekten soll Patienten dazu führen, ihre Vorerfahrungen optimalerweise nicht mehr unbewusst, unreflektiert und generalisiert anzuwenden, sondern realitätsgerechter und flexibler auf ihre Umwelt und deren unterschiedliche Anforderungen zu reagieren. Mit der ZBKT-Methode nach Luborsky liegt ein Instrument vor, mit dem Übertragung greifbar und für die therapeutische Arbeit nutzbar wird.

\subsection{Die generalisierte Angststörung - Entstehungsmodell und Empirie}

Ursächlich wird von einem Zusammenwirken einer genetischen Veranlagung zur „Ängstlichkeit“ (Vulnerabilität) mit gewissen auslösenden Faktoren und aufrechterhaltenden Bedingungen ausgegangen (Becker und Margraf 2007). Dabei spielen eigene Erfahrungen im Umgang mit Angst und Bedrohung ebenso eine Rolle wie das Lernen am Modell, wie es zum Beispiel der Umgang der Eltern mit Sorgen, Ängsten und Bedrohungen darstellt. GASPatienten leiden definitionsgemäß mindestens 6 Monate an unkontrollierbaren Sorgen und weisen einen eher unspezifischen Symptomenkomplex bestehend aus Rastlosigkeit, Ermüdbarkeit, Konzentrationsschwierigkeiten, Reizbarkeit, Muskelspannung und Schlafstörungen auf (Hoyer et al. 2003).

GAS ist eine chronisch verlaufende Erkrankung, deren Lebenszeitprävalenz mit 5 - 6\% angegeben wird (Kessler et al. 2005). Eine große Stichprobe innerhalb der amerikanischen Bevölkerung zeigt auf, dass Frauen doppelt so häufig wie Männer von GAS betroffen sind. Da allerdings Frauen - im Gegensatz zu Männern - häufiger komorbide Erkrankungen aufweisen, macht es den Anschein, GAS komme bei Frauen häufiger vor (Wittchen et al. 1994). Maier et al. (2000) konnten in einer gemischt geschlechtlichen Patientengruppe, deren Hauptdiagnose als GAS ohne Komorbiditäten definiert war, kein unterschiedlich häufiges Vorkommen zwischen Männern und Frauen mehr nachweisen. Häufige komorbide Störungen von GAS sind andere Angststörungen, Persönlichkeitsstörungen und depressive Episoden (Becker und Margraf 2007). GAS beginnt häufig zwischen dem 20. und 30. Lebensjahr und zeigt bei Frauen einen zweiten Gipfel nach dem 45. Lebensjahr (Hoyer et al. 2003). Das macht sie zur häufigsten Angststörung im höheren Lebensalter, weshalb auch das Alter als Risikofaktor gewertet werden kann. Auch Witwertum, Scheidung und Arbeitslosigkeit korrelieren signifikant mit GAS (Wittchen et al. 1994).

Yonkers et al. (2000) zeigten im Rahmen einer prospektiven Studie des Harvard-Brown Anxiety Research Programs, dass nur 38\% der untersuchten GAS-Patienten nach fünf Jahren vollständig in Remission begriffen waren und GAS eine chronische Erkrankung mit schwankender Ausprägung der Symptome ist, die meist ein Jahrzehnt und länger anhält. Tonks (2003) hält eher die Remission als die Heilung der Erkrankung für ein realistisches, mit 
dem Patienten zu vereinbarendes Therapieziel. Bleibt die Erkrankung unbehandelt oder schreitet unter Therapie fort, so gilt sie als Risikofaktor zur Entwicklung einer Depression binnen zwei bis vier Jahren (Wittchen et al. 2001).

Das Primary Care Anxiety Project ist eine Longitudinalstudie in der hausärztlichen Primärversorgung Amerikas und umfasst insgesamt 539 angstgestörte Patienten. 135 (25\%) von ihnen erfüllen nach DSM IV die Kriterien einer GAS. Weisberg et al. (2007) untersuchten, welche Behandlung die Patienten von Hausärzten und welche von Psychiatern erhielten, worauf im Detail nicht weiter eingegangen werden soll. Die Studie sensibilisiert den Leser jedoch für die Hintergründe, warum Patienten eine Therapie verwehrt bleibt. Zwei der drei häufigsten Gründe sind das fehlende Bewusstsein für das Vorhandensein einer therapiebedürftigen Erkrankung und die fehlende Überzeugung für die Wirksamkeit von Pharmakotherapie bei seelischen Problemen. Die meisten Patienten gaben jedoch an, der Hausarzt habe die Möglichkeit einer Pharmakotherapie nicht erwähnt, weshalb letztlich fast die Hälfte der angstgestörten Patienten unbehandelt blieb.

Als Erklärung für das fehlende Bewusstsein für eine therapiebedürftige Erkrankung kann herangezogen werden, dass an GAS erkrankte Patienten im Falle eines Arztbesuches nur selten ein Beschwerdebild zeigen, bei welchem die Angst offen sichtbar im Vordergrund steht. Diesen Patienten sind Ängste weder ,in das Gesicht geschrieben“, noch berichten sie explizit von ihnen. Vielmehr leiden sie unter einem unspezifisch wirkenden Symptomenkomplex, was die Diagnosefindung für den Arzt erschwert und häufig dazu führt, dass die Schwere der Erkrankung lange verkannt wird (Margraf und Schneider 2009). Die hohe Bedeutung sensitiver diagnostischer Instrumente für den klinischen Alltag wird vor diesem Hintergrund deutlich.

Seit vielen Jahrzehnten gibt es zahlreiche Fremd- und Selbstbeurteilungsverfahren zur Erfassung von Angststörungen. Mit der Hamilton Angstskala (HAM-A) (Hamilton 1959) sei ein Fremdbeurteilungsverfahren genannt, mit dem DIA-X Fragebogen (Wittchen und Pfister 1997) ein Selbstbeurteilungsverfahren. Die Diagnosestellung der dieser Dissertation zugrundeliegenden Studie erfolgte über strukturierte, klinische Interviews (SKID I, II) (Wittchen et al. 1997) nach DSM IV, worauf im Folgenden eingegangen wird.

\subsection{Die generalisierte Angststörung - Diagnosestellung und Therapie}

Freud (1895/1992) sprach von einer „Angstneurose“ bestehend aus ängstlicher Erwartung und körperlichen Symptomen. Eine präzise Definition der generalisierten Angststörung (GAS) als eigenständige Diagnose konnte erst durch das diagnostische und statistische Manual psychischer Störungen (DSM) erreicht werden, welches 1952 durch die American Psychiatric Association (APA 1952) geschaffen wurde.

Das Manual liegt inzwischen in der revidierten vierten Version vor (DSM IV TR (TextRevision)). Per DSM III (1980) durfte die Diagnose GAS nur vergeben werden, wenn keine psychischen Komorbiditäten, wie Depressionen oder andere Angststörungen vorlagen. Die revidierte Version DSM III R (1987) definierte die GAS erstmals als eigenständige Kategorie, die das Sorgen als Hauptmerkmal der Erkrankung in den Mittelpunkt stellte. 1994 folgte die Version DSM IV in welcher Sorgen erstmals als exzessiv und unkontrollierbar umschrieben wurden. 2000 folgte die Version DSM IV TR (Text-Revision). Die Diagnose GAS trägt den Code DSM IV: 300.02. Für 2013 ist die Version DSM V geplant. 
Abb.1 Veränderungen der GAS-Diagnose im DSM-Klassifikationssystem

(Hoyer et al. 2003, S. 268)

\begin{tabular}{|c|c|c|c|}
\hline & DSM III (1980) & DSM III R (1987) & DSM IV (1994) \\
\hline $\begin{array}{l}\text { Zentrale } \\
\text { Merkmale }\end{array}$ & $\begin{array}{c}\text { Generalisierte } \\
\text { überdauernde Angst }\end{array}$ & $\begin{array}{l}\text { Unrealistische oder exzessive } \\
\text { Angst und Besorgnis } \\
\text { (Erwartungsangst) in } 2 \text { oder } \\
\text { mehr Lebensbereichen }\end{array}$ & $\begin{array}{c}\text { Exzessive und } \\
\text { unkontrollierbare Angst und } \\
\text { Besorgnis (Erwartungsangst) } \\
\text { bezüglich mehrerer } \\
\text { Lebensbereiche }\end{array}$ \\
\hline Dauer & Mindestens 1 Monat & Mindestens 6 Monate & Mindestens 6 Monate \\
\hline Symptome & $\begin{array}{l}\text { Symptome aus den } \\
\text { Kategorien: } \\
\text { 1.) Motorische } \\
\text { Anspannung } \\
\text { 2.) Vegetative } \\
\text { Übererregbarkeit } \\
\text { 3.) Erwartungsangst } \\
\text { 4.) Hypervigilanz, } \\
\text { erhöhte } \\
\text { Aufmerksamkeit }\end{array}$ & $\begin{array}{l}\text { Mind. } 6 \text { von } 18 \text { Symptomen aus } \\
\text { den folgenden Bereichen: } \\
\text { 1.) Motorische } \\
\text { Anspannung } \\
\text { 2.) Vegetative } \\
\text { Hyperaktivität } \\
\text { 3.) Hypervigilanz und } \\
\text { erhöhte } \\
\text { Aufmerksamkeit }\end{array}$ & $\begin{array}{l}\text { Mind. } 3 \text { von } 6 \text { Symptomen: } \\
\text { 1.) Rastlosigkeit } \\
\text { 2.) Leichte Ermüdbarkeit } \\
\text { 3.) Konzentrations- } \\
\text { schwierigkeiten } \\
\text { 4.) Reizbarkeit } \\
\text { 5.) Muskelspannung } \\
\text { 6.) Schlafstörungen }\end{array}$ \\
\hline $\begin{array}{l}\text { Ausschluss- } \\
\text { kriterien }\end{array}$ & $\begin{array}{l}\text { Andere Angst- oder } \\
\text { Affektive Störung }\end{array}$ & $\begin{array}{l}\text { Mittelpunkt der Sorge steht mit } \\
\text { einer anderen Störung der } \\
\text { Achse I in Verbindung } \\
\text { Sorgen treten ausschließlich im } \\
\text { Verlauf einer Stimmungs- oder } \\
\text { psychotischen Störung auf }\end{array}$ & $\begin{array}{l}\text { Mittelpunkt der Sorge steht mit } \\
\text { einer anderen Störung der } \\
\text { Achse I in Verbindung } \\
\text { Sorgen treten ausschließlich im } \\
\text { Verlauf einer Stimmungs- oder } \\
\text { psychotischen Störung auf }\end{array}$ \\
\hline
\end{tabular}

Auch in der von der Weltgesundheitsorganisation (WHO) etablierten „International Classification of Diseases“ (aktuell ICD 10-GM (German Modification) Version 2012 (DIMDI 2012)) wird die GAS geführt und trägt den Code ICD-10: F41.1. Auf die ICD-10Klassifikation wird nicht weiter eingegangen.

Nach Reimer et al. (2007) gilt zur Behandlung von Angsterkrankungen grundsätzlich das Vorrecht von Psychotherapie vor Pharmakotherapie. Psychotherapeutische Interventionen seien wesentlicher Bestandteil der Therapie. Pharmakotherapie werde nur primär angewendet, wenn aufgrund Ermangelung akut vorhandener Therapieplätze eine geeignete Psychotherapie zeitnah nicht verfügbar ist, oder die Schwere der Angststörung ein Einlassen auf die Therapie ohne Psychopharmaka nicht ermöglicht. Auch eine schwere depressive Störung im Sinne einer Komorbidität kann eine begleitende Pharmakotherapie erforderlich machen.

Für die GAS ist zum jetzigen Zeitpunkt noch nicht ausreichend geklärt, ob eine Kombinationsbehandlung aus Psychotherapie und Pharmakotherapie im Vergleich zur alleinigen Psychotherapie eine bessere Wirksamkeit zeigt (Reimer et al. 2007). Dennoch werden etwa 50\% der GAS-Patienten medikamentös behandelt (Wittchen et al. 1994).

Wenn eine medikamentöse Therapie indiziert ist, so sind nach den gängigen Lehrbüchern bei Angststörungen im Allgemeinen Antidepressiva das Mittel der ersten Wahl (Benkert und Hippius 2009, Janssen et al. 2006). Citalopram, ein selektiver SerotoninWiederaufnahmehemmer (SSRI) eignet sich als gängiges Antidepressivum besonders zur Therapie der über 60-jährigen Patienten (Lenze et al. 2005). Unter anderem zeigen Escitalopram und Paroxetin Langzeiteffekte in der Behandlung der generalisierten Angststörung (Bielski et al. 2005). Für Antidepressiva sind bekanntermaßen bereits zahlreiche 
Nebenwirkungen dokumentiert. Neben gastrointestinalen Symptomen, Gewichtszunahme und sexueller Dysfunktion, wovon letztere mit einer Prävalenz von ca. 40\% (Venlafaxin) (Clayton et al. 2002) während der Behandlung als hoch eingestuft werden kann, sind auch ein erhöhtes Blutungsrisiko (Movig et al. 2003) und die Abnahme der Knochendichte bei Frauen beschrieben (Williams et al. 2008), was die besondere Eignung des Präparates zur Therapie der über 60-jährigen in Frage stellt. Neben der antidepressiven und anxiolytischen Komponente liegt ihr Vorteil gegenüber Benzodiazepinen jedoch im fehlenden Abhängigkeitspotential, der Nachteil in der längeren Wirklatenz.

Benzodiazepine werden häufig in akuten Krisen oder zur Überbrückung der verzögert einsetzenden Wirkung von Antidepressiva eingesetzt. Als biologisch orientierte Psychiater betonen Benkert und Hippius (2009), dass gerade zu Beginn einer Psychotherapie, oder bei einer akuten Exazerbation auf eine Begleitmedikation mit einem Benzodiazepin zur Krisenintervention nicht verzichtet werden kann und sie Mittel der ersten Wahl ist. Bei einer über mehrere Monate bestehenden Angsterkrankung sollten Benzodiazepine nach 4-6 Wochen abgesetzt sein. Durch Benzodiazepine wird kein langanhaltender therapeutischer Effekt erzielt. Bekanntermaßen ist auch bei Benzodiazepinen die Liste der Nebenwirkungen lang und birgt vor allem bei älteren Patienten größere Risiken (Petrovic et al. 2003).

Bei den zur Behandlung der GAS eingesetzten psychotherapeutischen Verfahren handelt es sich vor allem um Verhaltenstherapie (VT). Seltener finden psychodynamische Therapieverfahren bei GAS-Patienten Anwendung, zumal ihre Wirksamkeit noch unzureichend erforscht ist (Leichsenring 2009). Leichsenring (2009) und Wiegand (2010) verglichen beide Therapieverfahren hinsichtlich ihrer Anwendbarkeit bei GAS-Patienten.

Dem Leitfaden der psychosomatischen Medizin und Psychotherapie (Janssen et al. 2006, S.51, S. 373-374) sind folgende Ausführungen zur VT zu entnehmen. Sie beinhaltet eine große, heterogene Gruppe von Ansätzen, wozu Kenntnisse der Lerntheorie, Sozialpsychologie, kognitiven Psychologie und Emotionspsychologie gehören. Gemeinsame Grundlage ist die Annahme, dass dem Erwerb oder der Veränderung von Verhalten oder Einstellungen Lernvorgänge vorausgehen. Somit kann die VT klinisch bei allen psychischen Störungen und auch vielen körperlichen Erkrankungen - sei es als primäre oder ergänzende Therapie - eingesetzt werden. Die VT ist sowohl problem-, ziel- und handlungsorientiert, als auch transparent und nicht auf das therapeutische Setting begrenzt. Des Weiteren soll sie auch Hilfe zur Selbsthilfe sein. Durch das Erlernen neuer Verhaltens- und Lernprinzipien sollen problematische Verhaltensweisen beeinflusst und therapiert werden.

Zu diesen Verhaltens- und Lernprinzipien zählen zum Beispiel:

1. Systematische Desensibilisierung von Phobien durch vorgestellte Angstreize oder durch Konfrontation in der Realität

2. Flooding - Angstpatienten werden in der Realität mit massiven Angstreizen konfrontiert, durch Habituation erlischt die Angstreaktion schließlich

3. Operantes Lernen - durch Belohnung und Bestrafung werden dysfunktionales Verhalten abgebaut und neue Verhaltensstrategien erworben

4. Lernen am Modell - durch Beobachtung, Nachahmung und Übernahme von komplexen Verhaltensweisen vorbildhafter Personen in das eigene Verhaltensrepertoire. 
Als Beispiel innerhalb der praktischen, therapeutischen Arbeit mit GAS-Patienten sei das kognitiv behaviorale Behandlungsmanual genannt (Leibing et al. 2003), welches auch in der Behandlung der VT-Patienten der vorliegenden Studie Anwendung findet.

Es beinhaltet folgende grundlegende Komponenten:

1. Sammlung und Vermittlung von Informationen

2. Erarbeitung eines plausiblen Störungsmodelles

3. Beginn des Aufbaus einer tragfähigen Therapeut-Patient-Beziehung

4. Erlernen des Entspannungsverfahrens

5. Reduktion von Sorgen

6. Abbau kognitiver Verzerrungen und dysfunktionaler Kognitionen

7. Abbau des Vermeidungs- und Rückversicherungsverhaltens

8. Aufbau von Bewältigungsstrategien und Förderung von angstinkompatiblen Aktivitäten

9. Rückfallprophylaxe.

Nach Jansen et al. (2006, S. 389) gelten als methodische Grundprinzipien der psychodynamischen Therapieverfahren:

1. Fokussierung auf aktuelle Probleme und Beziehungen in den psychosozialen Situationen

2. Bearbeitung der reaktualisierten Beziehungsrepräsentanzen in den aktuellen Beziehungen, also auch in der zum Therapeuten

3. Dezentrierung des Übertragungsraumes, keine Zentrierung der Übertragung auf den Therapeuten allein, Gleichstellung der Übertragung auf den Therapeuten mit den Übertragungen in anderen Beziehungen

4. Aktive therapeutische Haltung: Neben Phasen der freien Assoziation treten thematische Phasen mit Zentrierung auf definierte Konflikte

5. Grundsätzliche Aufrechterhaltung technischer Neutralität, jedoch verstärkt supportive Interventionen wie Unterstützung, Ermutigung, Grenzsetzungen und eine selektive Abstinenz

6. Steuerung und Einschränkung der Regression

7. Handlungsaktivierende Interventionen und Unterstützungen bei Strukturstörungen

8. Ziel ist eine Konfliktlösung, Einsicht, Erleben von Akzeptanz und Identifizierung mit der annehmenden Haltung des Therapeuten.

Basierend auf der Supportiv-Expressiven Therapie (SET) nach Luborsky (1999) entwickelten Leichsenring et al. (2005) eine psychoanalytisch orientierte Fokaltherapie zur Behandlung von GAS. Sie soll als Beispiel der praktischen, therapeutischen Arbeit genannt werden. Sie ist eine ambulante Kurzzeittherapie, deren zentraler Bestandteil die Formulierung eines ZBKT`s ist, anhand dessen aktuelle und vergangene Beziehungen aufgearbeitet und aus der neue Handlungsstrategien im Umgang mit Wünschen und Reaktionen abgeleitet werden sollen. Hierzu werden supportive (stützende) und expressive (deutende) Interventionen genutzt. Die psychodynamisch therapierten Patienten, deren Daten in dieser Dissertation ausgewertet wurden, erhielten eine solche manualisierte Fokaltherapie. Da das Bestimmen und Verstehen des ZBKTs zentraler Bestandteil dieses Therapieverfahrens ist, ist die manualisierte Fokaltherapie hinsichtlich unserer Fragestellungen optimal geeignet. 


\subsection{Fragestellung und Zielsetzung}

Die ZBKT-Methode wurde bereits an verschiedenen Patientengruppen angewendet. Zu nennen sind hier u.a. die Bestimmung der ZBKT`s bei Patienten mit depressiven Störungen (Vanheule et al. 2006), Patienten mit Borderline-Persönlichkeitsstörungen (Drapeau und Perry 2004) und Patienten mit Borderline-Persönlichkeitsstörung mit und ohne Suizidversuch (Chance et al. 2000). Auch bei GAS-Patienten wurden ZBKT's bestimmt (Crits-Christoph et al. 1995).

Die ZBKT-Methode zeigt eine mehrfach nachgewiesene gute IRR. Da die Auswertungsverfahren und Kategoriensysteme zur Bestimmung des ZBKT stets weiterentwickelt wurden, soll über den Vergleich der Höhe der IRR auf Clusterebene abgeleitet werden, ob ein Kategoriensystem zur Bestimmung des ZBKT bei GAS-Patienten $\mathrm{zu}$ bevorzugen ist.

Die Forschungsergebnisse zur ZBKT-Methode beziehen sich weitestgehend auf psychoanalytisch orientierte Therapieverfahren. Offen bleibt, ob sich Übertragungsaspekte, die mit der ZBKT-Methode erfasst werden sollen, vergleichbar deutlich darstellen, wenn Narrative aus anderen Quellen, zum Beispiel verhaltenstherapeutischen Sitzungen, stammen. Albani et al. (2008, S. 137) und Luborsky (1999, S. 166) fordern daher vergleichende Untersuchungen zur ZBKT-Methode innerhalb unterschiedlicher Behandlungsgruppen.

Leichsenring et al. (2009), Werling (2010) und Wiegand (2010) verglichen VT- und SETVerfahren bei GAS-Pateinten. Leichsenring et al. (2009) dokumentierten, dass GAS-Patienten von VT und SET gleichermaßen profitieren. Die vergleichenden Untersuchungen sind von Bedeutung, da GAS-Patienten bislang hauptsächlich verhaltenstherapeutisch behandelt werden. Weitere Untersuchungen müssen folgen, um die Anwendbarkeit von psychodynamischen Verfahren für GAS-Patienten zu belegen.

Zudem stellt sich die Frage, ob VT- und SET-Patienten mit derselben Grunderkrankung unterschiedliche zentrale Themen aufweisen und ob die Behandlungsmethode Einfluss auf das hat, was erzählt wird. Findet sich gar ein übergeordnetes, zentrales Thema, welches allen GAS-Patienten gemein ist? Wo liegen diese Gemeinsamkeiten, wo die Unterschiede zu anderen Patientengruppen? Wie entwickeln und verändern sich zentrale Themen von VT- und SET-Patienten im Therapieverlauf? Der Vergleich maßgeschneiderter und ausgezählter ZBKT`s soll unsere Beobachtungen abrunden.

In insgesamt fünf Schritten werden nun die zentralen Fragen der vorliegenden Dissertation erschlossen. In selbiger Reihenfolge werden sie im Ergebnis- und Diskussionsteil behandelt. 
1.) Kann aus dem direkten Vergleich der durch verschiedene Kategoriensysteme bestimmten Inter-Rater-Reliabilität (IRR) auf Clusterebene eine Tendenz zur Bevorzugung eines Kategoriensystems zur Bestimmung des ZBKT abgelesen werden? Darf eine relativ kurze Schulungsdauer zum Erlernen der ZBKT-Methode als ausreichend eingestuft werden?

Zur Bestimmung zentraler Themen von Patienten mit der Hauptdiagnose GAS wurden drei Auswertungssysteme angewendet:

1. Klassische ZBKT-Standardkategorien und Cluster (Barber et al. 1990)

2. Alternative Cluster basierend auf den ursprünglichen Standardkategorien (Körner et al. 2002)

3. Reformulierte Standardkatagorien/Cluster (Albani et al. 2002 a,b).

Sie wurden unter den realistischen Bedingungen einer klinischen Studie miteinander verglichen. Die Bemessung der Inter-Rater-Reliabilität (IRR) spielte eine zentrale Rolle, da sie dazu dient, die klinische Brauchbarkeit und Anwendbarkeit der Kategoriensysteme unter der Voraussetzung zu belegen, dass eine Anzahl zufällig ausgewählter Narrative von unabhängigen Beurteilern bearbeitet und somit eine objektivierte Darstellung der Qualität der Kategoriensysteme hinsichtlich der Reproduzierbarkeit der Ergebnisse möglich wird. Mit einer Gegenüberstellung der originalen und überarbeiteten SK und CL der drei Auswertungssysteme sollen die beschriebenen Differenzen und Eigenheiten der einzelnen Systeme transparent gemacht und somit ein Beitrag zur Frage geleistet werden, ob eines der drei Kategoriensysteme zur Ermittlung des ZBKT`s bevorzugt werden sollte. Hierzu wurde der direkte Vergleich der ermittelten IRR auf Clusterebene im Auszählverfahren (AV) genutzt.

Durch die Verwendung der rSK nach Albani et al. (2002a,b) und der aCL nach Körner (2002) wurde aufgrund der beschriebenen Mängel der klassischen SK und CL (Barber et al. 1990) eine höhere IRR erwartet. Die Höhe der IRR gilt als Maß für die klinische Brauchbarkeit der Kategorien.

Eine weitere Frage bezieht sich auf die Rahmenbedingungen der Schulung zum Erlernen der ZBKT-Methode. Insbesondere wurden die relativ kurze Schulungszeit und der Laienstatus der Untersucher kritisch hinterfragt. Als Medizinstudenten waren die Rater zuvor nicht mit psychotherapeutischer Arbeit in Kontakt gekommen. Ist die Methode für ,jedermann“ erlernbar?

2.) Gibt es ein spezifisches ZBKT angstgestörter Patienten? Lässt es sich mit dem anderer Patientengruppen vergleichen?

Vorhaben der weiteren Untersuchungen war es, die zentralen Beziehungskonfliktthemen aller Patienten zu erstellen und $\mathrm{zu}$ hinterfragen, ob sich für Patienten mit Generalisierter Angststörung ein übergeordnetes zentrales Thema finden lässt. Wenn sich ein zentrales Thema für Angstpatienten finden sollte, inwiefern wäre es dann mit dem ZBKT anderer Patientengruppen vergleichbar? Wo liegen die Unterschiede zwischen den Patientengruppen, was ist für welche Patientengruppe zentral? 
3.) Gibt es hinsichtlich zentraler Themen Unterschiede zwischen verhaltenstherapeutisch und psychodynamisch-tiefenpsychologisch behandelten GAS-Patienten?

Zur Beantwortung der dritten Frage ergeben sich folgende untergeordnete Fragestellungen: Welche Rolle spielt die Behandlungsform der Patienten? Hat sie Einfluss auf das Erzählen des Patienten? Stellen sich Patienten auf das ein, worauf ihre Therapeuten die Aufmerksamkeit richten?

König (1986/2000, S. 16-22) erklärt das Konzept vom steuernden Objekt und stellt die These auf, dass ,allen Patienten mit Angstsymptomatik ein spezifischer Umgang mit Objekten gemein... “" (sei): ,ihr Umgang mit Menschen und Dingen, die ihnen Menschen ersetzen, wird durch die Suche nach steuernden Funktionen der Objekte gekennzeichnet. Dem steuernden Objekt sind Ich-Funktionen, welche der Kompetenz einer sozial adäquaten Realitätsbewältigung dienen, zugeordnet. "

Werden den Therapeuten steuernde Funktionen zugeordnet? Wenn ja, fallen die Steuerungswünsche bei VT- und SET-Patienten unterschiedlich aus? Um dieser Frage nachzugehen, wurden die Patienten der Studie in eine VT- und eine SET-Gruppe unterteilt und das ZBKT separat für diese Therapiegruppen bestimmt und verglichen.

Luborksy (1999, S. 166) sieht im „Durcharbeiten“ des „immer wieder auftretenden Beziehungsmusters das entscheidende Mittel der psychotherapeutischen Veränderung. Deshalb sollte im Verlauf und zum Abschluss von analytischen Psychotherapien hauptsächlich diese Variable gemessen werden, um bestimmen zu können, inwieweit in der Therapie auf das zentrale Thema fokussiert worden ist und inwieweit Veränderungen innerhalb dieses Themas eingetreten sind. Insofern wäre es von beträchtlichem Wert herauszufinden, ob andere Psychotherapieformen ähnliche Änderungen im ZBKT erreichen.“ Mit dieser Dissertation wird ein Beitrag zur Beantwortung dieser Frage Luborskys geleistet.

4.) Verändern sich zentrale Themen von VT- und SET-Patienten während des Therapieverlaufes?

Mit der Bestimmung der ZBKT`s zu verschiedenen Therapiezeitpunkten (Therapiebeginn und Therapieende) wurde untersucht, ob sich zentrale Themen von verhaltenstherapeutisch- und psychodynamisch behandelten Patienten verändern und wenn ja, ob sie dies gleichermaßen oder unterschiedlich tun. Wo liegen die Unterschiede?

5.) Gibt es Unterschiede zwischen den maßgeschneiderten ZBKTs von VT- und SETPatienten?

Finden sich Unterschiede in den maßgeschneiderten ZBKT`s verschiedener Patientengruppen? Verändern sich TM-ZBKT`s anders als AV-ZBKT`s im Therapieverlauf? Was ist VT- was SET-typisch? 


\section{Material und Methoden}

Die Darstellung des Vorgehens zur Ermittlung zentraler Beziehungskonfliktthemen orientiert sich streng am Manual zur ZBKT-Methode (Luborsky et al. 1991). Um ZBKT`s von Patienten formulieren zu können, müssen Narrative aus Therapiestunden gewonnen werden. In den Narrativen werden Wünsche und Reaktionen erfasst und als Beziehungsepisode (BE) dokumentiert. Patienten berichten von Beziehungen und Interaktionen mit anderen Menschen, oder auch mit und zu sich selbst.

Das auszuwertende Material umfasste 171 Therapiesitzungen mit insgesamt 802 BE.

- 78 Sitzungen SET $=439 \mathrm{BE}$

- 93 Sitzungen VT $=363 \mathrm{BE}$

- Zum Zeitpunkt T1 (3. und 5. Stunde) 394 BE (212 SET, 182 VT)

- Zum Zeitpunkt T2 (17. und 18. Stunde) 408 BE (227 SET, 181 VT).

Die Therapiesitzungen waren bis zu 60 Minuten lang, das Material lag im Audioformat vor. Da nur die entsprechenden Textstellen mit den Narrativen und nicht das gesamte Material transkribiert wurden, war der Arbeitsaufwand einigermaßen kalkulierbar. Die Rater wurden über die Dauer von zwei Hochschulsemestern im Umgang mit der ZBKT-Methode geschult und erlangten sowohl in Gruppen- als auch in Einzelarbeit Routine im Umgang mit der Methode. Sie erlernten, bei der Zuordnung der SK und CL sinnvoll abzuwägen, sich in die Thematik des Patienten einzufühlen und Idiosynkrasien zu erkennen.

Da Patienten naturgemäß eine verschieden hohe Anzahl an Narrativen in die Auszählung einbringen und bei der Darstellung der häufigsten CL somit formal die Gefahr einer ungleichen Gewichtung einzelner CL entstehen würde, wurde der prozentuale Anteil jedes CL pro Patient errechnet und für die Anzahl der in die Auswertung eingehenden Patienten gemittelt.

\subsection{Beziehungsepisoden und ZBKT-Komponenten}

BE`s enthalten in der Regel einen zumeist implizit geäußerten Wunsch des Erzählers (W), eine Reaktion des Objektes (RO), auf welches sich bezogen wird, und wiederum eine Reaktion des Erzählers, des Subjektes (RS), auf die RO.

Um eine ausreichende Anzahl an BE`s zur Bestimmung des ZBKT (10 oder mehr) zu erhalten, wurden zu Beginn und Ende der Therapie jeweils zwei Therapiestunden eines Patienten zusammengefasst und ausgewertet. Die Anzahl der erfassten BE`s war je nach Behandlungsmethode verschieden hoch. Psychodynamische Therapiestunden waren erwartungsgemäß reicher an BE`s.

Den Beurteilern war zum Auswertungszeitpunkt nicht bekannt, ob es sich um Sitzungen des Therapiebeginn oder -endes handelte, ebenso wenig waren persönliche Angaben zum Patienten (Alter, Ausbildungsstand, etc.) bekannt. Die Entschlüsselung der Daten erfolgte erst nach Abschluss der Auswertung, wodurch eine Beeinflussung der Beurteiler hinsichtlich ihrer Einschätzungen verhindert werden sollte. 


\subsection{Vollständigkeit von Beziehungsepisoden}

Wichtigstes Kriterium einer BE ist deren Vollständigkeit hinsichtlich der ZBKTKomponenten. Die Bewertung der Vollständigkeit führt letztlich die Entscheidung für oder gegen die Verwendung der Episode zur Transkription und Auswertung herbei. Auf einer Skala von 1 (sehr unvollständig) bis 5 (detailliert) sollten nur BE`s mit einer Wertigkeit ab 2,5 in die Auswertung einbezogen werden. Geeignet sind Erzählungen, in denen der Patient sehr ausführlich über die Interaktionen mit anderen Menschen berichtet, die Erzählung mit Details ausschmückt und es dem Betrachter somit ermöglicht, sich in die Situation eines Regisseurs zu versetzen, der sich die Erzählung als Filmszene vorstellt. Je plastischer die Szene wirkt, desto vollständiger wird die BE gewertet.

Abb. 2 Bewertung der Vollständigkeit von Beziehungsepisoden (Luborsky et al. 1991, S.7)

\begin{tabular}{|l|l|}
\hline Bewertung & Inhalt der BE \\
\hline 1 & Ich traf Joe und wir unterhielten uns (keine ZBKT-Komponenten) \\
\hline 1,5 & $\begin{array}{l}\text { Ich traf Joe, wir unterhielten uns, aber er sagte wenig (Eine ziemlich } \\
\text { unbestimmte Reaktion des Objekts) }\end{array}$ \\
\hline 2 & $\begin{array}{l}\text { Ich traf Joe, wir unterhielten uns, aber er sagte wenig. Er ist ein alter } \\
\text { Schulfreund, den ich mag (Mehrere unvollständige Komponenten, ein Hinweis } \\
\text { auf einen Wunsch und eine Reaktion des Selbst) }\end{array}$ \\
\hline $\mathbf{2 , 5}$ & $\begin{array}{l}\text { Ich traf Joe, wir unterhielten uns, aber er sagte wenig. Er ist ein alter } \\
\text { Schulfreund, den ich mag. Ich war enttäuscht, dass er kaum von unseren } \\
\text { früheren gemeinsamen Erlebnissen sprach (Genügend Informationen, um } \\
\text { Wunsch, Reaktion des Objekts und Reaktion des Subjekts zu kodieren) }\end{array}$ \\
\hline 3 & $\begin{array}{l}\text { Bei der Bewertung >2,5 beruhen die Bewertungen der Vollständigkeit darauf, } \\
\text { wie genau der Patient die Geschichte erzählt und wie detailliert die } \\
\text { Informationen für jede der Komponenten sind }\end{array}$ \\
\hline 4 & $\begin{array}{l}\text { Ich traf Joe und wir unterhielten uns, aber er sagte wenig. Er ist ein alter } \\
\text { Schulfreund, den ich mag. Ich war enttäuscht, dass er kaum von unseren } \\
\text { früheren gemeinsamen Erlebnissen sprach. Ich versuchte, die vergangene Zeit } \\
\text { wieder zu erleben und so ein Gefühl wie damals zu empfinden, aber Joe wirkte } \\
\text { zerstreut. Ich schlug vor, dass wir uns nächste Woche zum Essen treffen und er } \\
\text { stimmte zu (Alle drei Komponenten sind detaillierter und ausführlicher) }\end{array}$ \\
\hline 5 & \begin{tabular}{l} 
Wie in der Beschreibung für 4 aber noch mehr Details \\
\hline
\end{tabular}
\end{tabular}

\subsection{Bestimmung von Hauptperson und Zeitebene}

Die Bestimmung der Hauptperson einer BE ist nicht immer eindeutig. Häufig finden mehrere Objekte im Narrativ Erwähnung. Es handelt sich dann entweder um eine Personengruppe, die z.B. als „Familie“ oder „Freunde“ zusammengefasst werden kann, oder aber es spielen mehrere Personen eine Rolle. Der Beurteiler muss eine einzige Person als Hauptperson definieren, wenn die Personen nicht als Gruppe zusammengefasst werden können. Erscheint es unmöglich, sich auf eine einzige Hauptperson oder Personengruppe festzulegen, so muss die $\mathrm{BE}$ in mehrere Unterepisoden mit jeweils verschiedenen Beziehungsobjekten unterteilt 
werden. Die Bestimmung der Hauptperson ist wichtig und sollte genau sein, da sich die ZBKT-Komponenten (W, RO, RS) auf diese Hauptperson beziehen.

Narrative können weiterhin einer bestimmten Zeitspanne zugeordnet werden, was für das spätere ZBKT jedoch nicht unbedingt notwendig ist. Narrative aus der Vergangenheit wurden mit dem Buchstaben „V“ versehen. Da die Vergangenheit häufig eine sehr große Zeitspanne umfasst, wird sie zusätzlich in die Vergangenheit der Kindheit (bis 10 Jahre), der Jugend (1118 Jahre) und der Adoleszenz (ab 18 Jahre) unterteilt. Erzählungen aus der Gegenwart werden mit „G“ gekennzeichnet. Die Bezeichnung „Gegenwart“ definiert den Zeitraum der vergangenen vierzehn Tage bis zur gegenwärtigen Therapiestunde. Narrative, die zeitlich nicht einzugrenzen sind, also weder der Gegenwart noch der Vergangenheit zugeordnet werden können, werden als ,generalisiert“ bezeichnet. Sie beschreiben keine konkrete Situation und werden nicht in die Auswertung einbezogen, da sie dem Zuhörer bereits eine mehr oder weniger reflektierte Generalisierung von Beziehungserfahrungen vermitteln sollen.

\subsection{Erstellen des Therapie-Transkriptes}

Die Tonbandaufzeichnungen der Therapiestunden wurden zweimal gehört. Während der erste Vorgang dazu diente, Narrative und BE`s zu identifizieren und auf Vollständigkeit zu prüfen, wurden sie im zweiten Durchgang erneut evaluiert und gegebenenfalls aussortiert. Das erneute Hören des Tonbandes, wenngleich es auch zeitaufwendig war, erleichterte das Erkennen von BE`s, oder auch wichtiger inhaltlicher Zusammenhänge. Vollständige BE`s wurden erst im zweiten Vorgang transkribiert, nummeriert und zur genauen Dokumentation mit der entsprechenden Zeitebene und dem Beziehungsobjekt gekennzeichnet. Das Vorgehen war praktikabel und der zeitliche Aufwand kalkulierbar, da lediglich die BE`s und nicht das gesamte Tonband transkribiert werden mussten.

\subsection{Zuordnung zu Standardkategorien und Clustern}

Sobald die BE`s transkribiert waren, folgte die inhaltliche Zuordnung zu den SK und CL der Auswertungssysteme. Wir verwendeten die Auswertungssysteme von Barber et al. (1990), Körner et al. (2002) und Albani et al. (2002 a,b).

Barber et al. (1990) halten 34 SK für W und jeweils 30 SK für RO und RS bereit, welche sich schließlich zu jeweils 8 CL verdichten. Körner et al. (2002) entwickelten für die bekannten SK entsprechende aCL. Jeweils 10 aCL für W und RS, sowie 8 aCL für RO. Albani et al. (2002 a,b) stellten die Prädikate des reformulierten ZBKT-Systems auf. Sie lösten sich dabei von den herkömmlichen CL und entwickelten eine alphabetisch aufgestellte ClusterSammlung, welche sich in harmonische und disharmonische Komponenten mit hierarchischer Gliederung unterteilt.

Eine Besonderheit der Auswertung der vorliegenden Dissertation ist, dass wir die rSK sowohl in ihrer originalen Form (Albani (o): A 1.1 - M 3.4), als auch in einer vereinfachten Form (Albani (v): A-M ohne Subkategorien) verwendeten. Mit den vereinfachten Albani Kategorien (Albani (v)) wurden erwartungsgemäß höhere Inter-Rater-Reliabilitäten erzielt, für den Ergebnisteil wurden nur die Albani (v)-Kategorien verwendet. 
Zur vereinfachten Bearbeitung und Zuordnung der BE`s zu den SK und CL wurden 3 Auswertungsbogen erstellt:

\section{Auswertungsbogen Nr. 1}

Die ermittelten W-, RO- und RS-Komponenten wurden in möglichst textnaher Kurzform auf den Auswertungsbogen übertragen und zunächst den SK und CL von Barber et al. (1990) und Körner et al. (2002) zugeteilt. Hauptperson und Zeitebene wurden vermerkt.

Bei explizit geäußerten Wünschen bedurfte es bei der Übertragung nur eines geringen Abstraktionsvermögens. Sie wurden so textnah wie möglich übernommen. Etwas anders verhielt es sich mit implizit geäußerten Wünschen. Hierbei bedurfte es einer Abstraktionsebene, auf welcher möglichst unter Verzicht auf Interpretation die Wunschformulierung entstehen sollte. Es handelte sich somit eher um eine inhaltlich nahe Schlussfolgerung aus den tatsächlich geäußerten Erzählungen des Patienten. RO- und RSKomponenten waren in der Regel leichter zu identifizieren als W-Komponenten.

\section{Auswertungsbogen Nr. 2}

W-, RO- und RS-Komponente, Hauptperson und Zeitebene wurden auf den zweiten Auswertungsbogen übertragen. Zuvor wurde die Unterscheidung der Komponenten in WO und WS, sowie die der Reaktionskomponenten in Positive und Negative (PRO, NRO, PRS, NRS) vorgenommen. Dann erfolgte die Zuordnung zu den reformulierten SK von Albani et al. (2002 a,b).

Mit Hilfe des ersten und zweiten Auswertungsbogens ließ sich bereits das ZBKT im AV auf Clusterebene ablesen. Die Narrative der Therapiestunden am Therapiebeginn und der Stunden am Therapieende wurden zusammengefasst.

\section{Auswertungsbogen Nr. 3}

Der auch als Zusammenfassungsbogen bezeichnete dritte Auswertungsbogen diente dazu, maßgeschneiderte (engl.: tailormade, TM) Formulierungen zu finden. Auch für das TMZBKT wurden die beiden Stunden am Therapiebeginn und -Ende zusammengefasst. 


\subsection{ZBKT im Auszählverfahren vs Tailormade-ZBKT}

Liest man die häufigsten, in Cluster übersetzten W-, RO- und RS-Kategorien ab, so erhält man das ZBKT im AV auf Clusterebene. Es wurde für die klassischen ZBKT-CL (Barber et al. 1990), die aCL nach Körner (Körner et al. 2002) und die rSK nach Albani (Albani et al. 2002 a,b) bestimmt.

Durch das Auszählen der absoluten Häufigkeiten repräsentiert das ZBKT im AV quantitative Aspekte. Wir verglichen das ZBKT des Gesamtdatenpools (45 Patienten, davon 25 VT- und 20 SET-Patienten) mit den separat erstellten ZBKT`s der VT- und SET-Untergruppe.

Um erwartete Veränderungen und Entwicklungen im Therapieverlauf darstellen zu können, wurden die ZBKT's zu verschiedenen Therapiezeitpunkten (Therapiebeginn: 3.+5.Stunde = T1, Therapieende: 17.+18. Stunde $=$ T2) bestimmt und miteinander verglichen. Dabei wurde auf Unterschiede zwischen der VT- und SET-Gruppe geachtet.

Das TM-ZBKT versucht gegenüber dem quantitativen Aspekt des AV-ZBKT`s die qualitative Bedeutung einzelner Wünsche oder Reaktionen zusammenzufassen. Die ursprünglichen W-, RO- und RS-Komponenten der Narrative werden inhaltlich sortiert, um für ähnlich anmutende Wünsche und Reaktionen gemeinsame, maßgeschneiderte Formulierungen und somit den oft beschriebenen „Roten Faden“ finden zu können. So entstehen maßgeschneiderte zentrale Beziehungskonfliktthemen, welche nah an den Worten der Patienten und dennoch auf einer Abstraktionsebene liegen. Die Abstraktionsebene ist so gewählt, dass die Formulierung für die meisten Narrative einer Therapiestunde zutrifft. Das maßgeschneiderte ZBKT wird aufgrund der Nähe zur ursprünglichen Patientenaussage favorisiert. Für jeweils 10 VT- und SET-Patienten unserer Patientengruppe wurden maßgeschneiderte ZBKT`s bestimmt und miteinander verglichen.

\subsection{Bestimmung der Interrater-Reliabilität auf Clusterebene}

Die Höhe der IRR spiegelt die Verlässlichkeit wider, mit der zwei Beurteiler eine möglichst gute Übereinstimmung in der Auswahl und Zuordnung von SK und CL erzielen. Sie soll dazu dienen, die klinische Brauchbarkeit und Anwendbarkeit der Kategoriensysteme zu belegen. Zufällig ausgewählte therapeutische Sitzungen werden von verschiedenen Beurteilern bearbeitet, über die Höhe der IRR werden sowohl Qualität als auch Reproduzierbarkeit der Ergebnisse objektiv dargestellt.

Für diese Dissertation wurde die IRR auf der Clusterebene bestimmt. Sie wurde an 12 zufällig aus dem Gesamtdatenpool ausgewählten und von 3 unabhängigen Ratern bearbeiteten Therapiestunden ermittelt. Die Angabe der IRR erfolgte sowohl prozentual als auch mit dem Kappa-Koeffizienten. Das Cohens Kappa wurde 1960 von Jacob Cohen als statistisches Maß etabliert. Wir gingen von einer Berechnung der IRR zwischen zwei Ratern aus. Alle drei Rater wurden miteinander verglichen, sodass sich 3 Interrater (IR)-Paare bildeten.

Alle erhobenen Werte (n) wurden auf eine Kontingenztabelle aufgetragen. Die Summe aller auf der Diagonale aufgetragenen Werte ergab die Anzahl an Übereinstimmungen zwischen zwei Ratern. Alle außerhalb der Diagonalen aufgetragenen Werte entsprachen abweichenden Beurteilungen. 


$$
\kappa=\frac{p_{0}-p_{c}}{1-p_{c}}
$$

p0 (Übereinstimmung zwischen zwei Ratern) wird berechnet als:

$\mathrm{p} 0=$ Summe der Diagonalen $\sum / \mathrm{n}$

pc (erwartete, zufällige Übereinstimmung) wird berechnet als

$\mathrm{pc}=$ Summe fi $\mathrm{x}$ fi $/ \mathrm{n}^{2}$

fi x fi entsprach dem Produkt von Zeilensumme und Spaltensumme, welches addiert und ins Verhältnis zum Quadrat der Gesamtsumme gestellt wird, wie es folgendes Beispiel zeigt:

Abb. 3 Beispielrechnung Interrater-Reliabilität

\begin{tabular}{|l|l|l|l|l|l|l|l|l|l|}
\hline WCL & 1 & 2 & 3 & 4 & 5 & 6 & 7 & 8 & \\
\hline 1 & & & & & & & & & 0 \\
\hline 2 & & & & & & & & & 0 \\
\hline 3 & & & 1 & & & & & & 1 \\
\hline 4 & & & & 1 & & & 1 & & 2 \\
\hline 5 & & & & & & & & & 0 \\
\hline 6 & & & 1 & & & & & 1 & 2 \\
\hline 7 & & & 1 & & & 1 & 4 & & 6 \\
\hline 8 & & & & & & & & 1 & 1 \\
\hline & 0 & 0 & 3 & 1 & 0 & 1 & 5 & 2 & $\Sigma$ \\
\hline
\end{tabular}

Beispielrechnung für Wunsch-Cluster mit $\mathrm{N}=12$, Summe der Diagonalen $\sum=7$ $\mathrm{p} 0=7 / 12=0,58$

$\mathrm{pc}=3+2+2+30+2 / 144=39 / 144=0,27$

$\mathrm{K}=0,58-0,27 / 1-0,27=0,31 / 0,73=0.42$

Der Kappa-Wert unserer Beispielrechnung $(K=0.42)$ entspricht einer mittelmäßigen (moderaten) Übereinstimmung (Landis und Koch 1977). 
Abb. 4 Cohens Kappa

Landis und Koch (1977, S.165)

\begin{tabular}{|c|l|}
\hline Cohens Kappa & \\
\hline $\mathrm{K}<0$ & schlechte Übereinstimmung (poor agreement) \\
\hline K 0-0.20 & etwas Übereinstimmung (slight agreement) \\
\hline K 0.21-0.40 & ausreichende Übereinstimmung (fair agreement) \\
\hline K 0.41-0.60 & mittelmäßige Übereinstimmung (moderate agreement) \\
\hline K 0.61-0.80 & beachtliche Übereinstimmung (substantial agreement) \\
\hline K 0.81-1.00 & fast vollkommene Übereinstimmung (almost perfect agreement) \\
\hline
\end{tabular}

\subsubsection{IRR im naturalistischen Verfahren}

Staats und Biskup (1996) stellten fest, dass die Umstände der Erhebung von Narrativen Einfluss auf die Art der Narrative haben. Sie führen zu unterschiedlichen Ergebnissen und zu unterschiedlich deutlichen Übertragungsmustern, was wiederum Einfluss auf die Ermittlung des ZBKT`s und die Höhe der IRR hat.

Für diese Dissertation stellten wir ein „Naturalistisches Verfahren“ (NV) einem „Standardisierten Verfahren“ (SV) gegenüber. Im NV war es jedem einzelnen Rater eigenverantwortlich überlassen, die Narrative aus der Audioversion der Therapiestunde zu transkribieren, die ZBKT-Komponenten in den BE`s zu benennen, sie den SK und CL zuzuordnen und das ZBKT zu bestimmen. Die IRR (Clusterebene) des NV lag deutlich unter der bisherigen IRR für die ZBKT-Methode, da sich automatisch Differenzen in der Auswahl der Narrative und der Festlegung der ZBKT-Komponenten ergaben. Die Bestimmung war rein exemplarisch und wurde nicht in die Auswertung mit einbezogen, da wir uns für den Ergebnisteil streng an den Vorgaben des Manuals zur ZBKT-Methode (Luborksy et al. 1991) orientierten.

Dennoch möchten wir die Ergebnisse des NV zur Veranschaulichung der Notwendigkeit eines SV präsentieren, um aufzuzeigen, wie die Umstände der Erhebung, speziell das Fehlen einer aufgearbeiteten Datengrundlage, Einfluss auf die Höhe der IRR nehmen. Wir betrachten die Ergebnisse des NV jedoch bewusst außerhalb des eigentlichen Ergebnisteiles.

Die Abbildungen 5-8 zeigen die im NV erhobene IRR für die verschiedenen Auswertungssysteme (Doktorand A, B, C: hieraus IR-Paar 1-3)

Abb. 5 IRR im NV (Barber)

\begin{tabular}{|l|c|c|c|}
\hline IRR Barber & IR-Paar 1 & IR-Paar 2 & IR-Paar 3 \\
\hline W & k.53 & k.29 & k.44 \\
\hline RO & $\mathrm{k} .52$ & $\mathrm{k} .46$ & $\mathrm{k} .32$ \\
\hline RS & $\mathrm{k} .4$ & $\mathrm{k} .29$ & $\mathrm{k} .36$ \\
\hline
\end{tabular}

Abb. 6 IRR im NV (Körner)

\begin{tabular}{|l|c|c|c|}
\hline IRR Körner & IR-Paar 1 & IR-Paar 2 & IR-Paar 3 \\
\hline W & k.45 & k.21 & k.55 \\
\hline RO & k.43 43.27 \\
\hline RS & k.55 & k.38 & k.31 \\
\hline
\end{tabular}


Abb. 7 IRR im NV (Albani (o))

\begin{tabular}{|l|c|c|c|}
\hline IRR Albani (o) & IR-Paar 1 & IR-Paar 2 & IR-Paar 3 \\
\hline W & k.31 & k.31 & k.34 \\
\hline RO & k.33 & k.23 & k.24 \\
\hline RS & k.41 & k.4 & k.4 \\
\hline
\end{tabular}

Abb. 8 IRR im NV (Albani (v))

\begin{tabular}{|l|c|c|c|}
\hline IRR Albani (v) & IR-Paar 1 & IR-Paar 2 & IR-Paar 3 \\
\hline W & k.41 & k.33 & k.51 \\
\hline RO & k.38 & k.32 & k.33 \\
\hline RS & k.49 & k.45 & k.53 \\
\hline
\end{tabular}

Albani (o): originale Form A1.1 bis M 3.4 / Albani (v): vereinfachte Form. A-M (ohne Subkategorien)

Die Höhe der IRR bewegt sich zwischen k3.1 bis k5.3 für W, zwischen k2.1 und k4.6 für RO und zwischen k2.4 und k5.5 für RS. Wir dokumentierten keine Ergebnisse, welche die Bevorzugung eines Kategoriensystems unterstützen würden.

\subsubsection{IRR im standardisierten Verfahren}

Das standardisierte Verfahren zur Bestimmung der IRR ist im Vergleich zum NV streng an die Vorgaben im Manual zur ZBKT-Methode von Luborsky et al. (1991) angelehnt. Die Narrative und ZBKT-Komponenten wurden zuvor festgelegt und markiert. Nach dem Zufallsprinzip wurden 12 Therapiestunden ausgewählt und darin 76 Narrative mit ZBKTKomponenten markiert. Somit war von standardisierten, einheitlichen Untersuchungsbedingungen auszugehen.

Für die spätere Auswertung wurde nur die vereinfachte Form der reformulierten AlbaniCluster LU(v) verwendet, die Ergebnisse der LU(o) Auswertung wurden vernachlässigt. Dem Ergebnisteil ist die Höhe der IRR des standardisierten Verfahrens zu entnehmen. Die IRR im standardisierten Verfahren liegt deutlich über der oben aufgezeigten IRR des naturalistischen Verfahrens und wird detailliert im Ergebnisteil offengelegt.

\subsection{Patientenkollektiv, Aufnahme- und Ausschlusskriterien}

Die Auswertung basiert auf Narrativen aus Therapiestunden von 45 ambulanten Patienten mit der Hauptdiagnose GAS. Die Patienten nahmen an einer randomisierten, kontrollierten klinischen Studie für Kurzzeittherapie teil, welche teilweise von der Deutschen Forschungsgemeinschaft (DFG; LE 1250/1-1/1-2) gefördert wurde.

Die Studie wurde in der Abteilung für Psychosomatik und Psychotherapie der Universität Göttingen von Mai 2001 bis Juni 2007 durchgeführt und war von der Ethik-Kommission der Medizinischen Fakultät der Georg-August- Universität Göttingen genehmigt.

Die Patienten wurden von psychologischen Psychotherapeuten und Ärzten überwiesen oder durch Informationen und Werbung über die Presse für die Studie gewonnen. 25 Patienten erhielten eine VT, 20 Patienten eine psychodynamisch orientierte Fokaltherapie (SET).

Alle Patienten wurden in strukturierten, klinischen Interviews (SKID I, II) (Wittchen et al. 1997) nach DSM IV diagnostiziert. Die Interviews wurden von trainierten und erfahrenen 
Interviewern vorgenommen. Die Diagnosen wurden in Übereinstimmung von mindestens zwei der trainierten und erfahrenen Interviewer getroffen.

Abb. 9 Allgemeine Angaben zum Patientenkollektiv

\begin{tabular}{|l|c|}
\hline Allgemeine Angaben & \\
\hline Durchschnittliches Alter & 43,46 Jahre \\
\hline Geschlecht w/m & $80 \% / 20 \%$ \\
\hline Beziehungsstatus & $82,2 \%$ Langzeitbeziehung \\
\hline
\end{tabular}

Abb. 10 Ausbildungsstand des Patientenkollektives

\begin{tabular}{|l|c|c|}
\hline Bildung & \% & N \\
\hline Keine Ausbildung & 11,1 & 5 \\
\hline Hauptschulabschluss & 15,5 & 7 \\
\hline Mittlere Reife & 42,2 & 19 \\
\hline Lehre & 64,4 & 29 \\
\hline Meistergrad & 0 & 0 \\
\hline Abitur & 42,2 & 19 \\
\hline Hochschulabschluss & 24,4 & 11 \\
\hline
\end{tabular}

Abb. 11 GAS und Komorbiditäten (1)

\begin{tabular}{|l|c|c|}
\hline Hauptdiagnose & \% & N \\
\hline GAS als einzige psychische Störung & 33,3 & 15 \\
\hline GAS assoziiert mit komorbiden* Störungen & 66,6 & 30 \\
\hline
\end{tabular}

*eine oder mehrere komorbide Störungen

Abb. 12 GAS und Komorbiditäten (2)

\begin{tabular}{|l|c|c|}
\hline Komorbide Störungen bei GAS* & \% & N \\
\hline Andere Angststörungen & 33,3 & 15 \\
\hline Depressive Störungen & 24,4 & 11 \\
\hline Anpassungsstörungen & 22,2 & 10 \\
\hline Zwangsstörungen & 11,1 & 5 \\
\hline Somatoforme Störungen & 6,6 & 3 \\
\hline
\end{tabular}

*bezogen auf Patientenkollektiv der Studie

Patienten im Alter von 18 bis 65 Jahren mit der Hauptdiagnose GAS wurden in die Studie aufgenommen. In Anlehnung an ADIS-R (Di Nardo et al. 1993) war die Hauptdiagnose definiert als die ,schwerste mentale Funktionsstörung“".

Zu den Ausschlusskriterien zählten:

1. Das Vorhandensein einer akuten, instabilen oder schweren Achse-III-Störung, also ein somatischer, medizinischer Krankheitsfaktor, der für eine erfolgreiche Therapie hinderlich sein könnte

2. Das Vorhandensein einer aktuellen oder vergangenen Krankengeschichte bezüglich Schizophrenie, bipolarer Störung oder Cluster-A-Achse-IIPersönlichkeitsstörung

3. Das Vorhandensein einer aktuellen oder vergangenen neurologischen Funktionsstörung 
4. Das Vorhandensein von Alkohol- oder Drogenabhängigkeit (oder Missbrauch dieser), Essstörungen, Depression oder Borderline-Persönlichkeitsstörung innerhalb der letzten 12 Monate

5. Patienten die sich aktuell in psychotherapeutischer oder psychopharmakologischer Behandlung befinden.

Abb. 13 Aufnahme- und Ausschlusskriterien der Studie

\begin{tabular}{|c|c|c|}
\hline \multicolumn{2}{|l|}{ Insgesamt gescreent: 231 Patienten } & \multirow[b]{2}{*}{ Gesamt N } \\
\hline Grund des Ausscheidens: & Verlust & \\
\hline $\begin{array}{llllll}\text { Aufnahmekriterien nicht } & \text { erfüllt } & \text { und } & / & \text { oder } \\
\text { Ausschlusskriterium erfüllt } & & & & \end{array}$ & 174 & 57 \\
\hline Beendigung der Therapie wegen Umzug & 4 & 53 \\
\hline $\begin{array}{l}\text { Beendigung der Therapie wegen Problem mit } \\
\text { Therapeut }\end{array}$ & 1 & 52 \\
\hline Akustischer Mangel der Tonbandaufnahme & $7 *$ & 45 \\
\hline
\end{tabular}

* Patienten wurden von der Auswertung ausgeschlossen, wenn beide Sitzungen des Therapiebeginns (prae = 3.+5. Stunde $=\mathrm{T} 1)$ oder beide Sitzungen des Therapieendes $($ post $=17 .+18 .=\mathrm{T} 2)$ aufgrund der akustischen Mängel nicht zu verwerten waren.

Abb. 14 Materialverlust durch akustischen Mangel der Tonbandaufnahme

\begin{tabular}{|c|c|c|c|c|}
\hline Patientenkollektiv N=45 & $\begin{array}{l}\text { Therapiestd. } \\
\text { Nr.3 }\end{array}$ & $\begin{array}{l}\text { Therapiestd. } \\
\text { Nr.5 }\end{array}$ & $\begin{array}{l}\text { Therapiestd. } \\
\text { Nr. 17 }\end{array}$ & $\begin{array}{l}\text { Therapiestd. } \\
\text { Nr. 18 }\end{array}$ \\
\hline 36 Patienten & $\mathrm{x}$ & $\mathrm{x}$ & $\mathrm{x}$ & $\mathrm{x}$ \\
\hline 7 Patienten * & $\sim$ & $\mathrm{x}$ & $\mathrm{x}$ & $\mathrm{x}$ \\
\hline 2 Patienten * & $\mathrm{x}$ & $\mathrm{x}$ & $\sim$ & $\mathrm{x}$ \\
\hline
\end{tabular}

$\mathrm{x}=$ Therapiestunde lag in guter Qualität im Audioformat vor und wurde in Auswertung einbezogen

*= Sofern drei Therapiestunden in guter Qualität vorlagen, wurden Patienten für die Auswertung berücksichtigt

= Therapiestunde aufgrund akustischer Mängel der Tonbandaufnahme von Auswertung ausgeschlossen 


\section{Ergebnisse}

\subsection{Die Interrater-Reliabilität für das ZBKT im Auszählverfahren}

Folgende Darstellung über die Höhe der IRR bezieht sich auf die erste allgemeine Fragestellung der Dissertation. Die Qualität der verschiedenen Klassifikationssysteme wird über die Höhe der IRR miteinander verglichen. Drei Rater wurden einander gegenübergestellt, sodass 3 IR-Paare entstanden. Die Übereinstimmung wird sowohl prozentual als auch als Kappa-Koeffizient angegeben. Die Höhe des Kappa-Koeffizienten wird mit Hilfe einer Klassifikation von Landis und Koch (1977) übersetzt.

Abb. 15 IRR (Paar 1)

\begin{tabular}{|l|c|c|c|c|c|c|}
\hline \multirow{2}{|c|}{ IRR (Paar 1) } & \multicolumn{2}{|c|}{ W } & \multicolumn{2}{c|}{ RO } & \multicolumn{2}{c|}{ RS } \\
\hline & Kappa & \% & Kappa & \% & Kappa & $\%$ \\
\hline Barber *1 & .77 & 83 & .76 & 79 & .75 & 80,3 \\
\hline Körner *2 & .79 & 83 & .66 & 71 & .75 & 80,3 \\
\hline LU (v)*3 & .92 & 93,4 & .87 & 89,5 & .84 & 87 \\
\hline LU (o)*4 & .8 & 79 & .8 & 81,6 & .8 & 83 \\
\hline
\end{tabular}

Abb. 16 IRR (Paar 2)

\begin{tabular}{|l|c|c|c|c|c|c|}
\hline \multirow{2}{*}{ IRR (Paar 2) } & \multicolumn{2}{|c|}{ W } & \multicolumn{2}{c|}{ RO } & \multicolumn{2}{c|}{ RS } \\
\hline & Kappa & \% & Kappa & \% & Kappa & \% \\
\hline Barber *1 & .66 & 71,1 & .65 & 72,4 & .73 & 79 \\
\hline Körner * & .63 & 68,4 & .58 & 64,5 & .73 & 77,6 \\
\hline LU (v)* & .67 & 73,7 & .67 & 71 & .78 & 81,6 \\
\hline LU (o)*4 & .52 & 57,9 & .59 & 61,8 & .68 & 72,4 \\
\hline
\end{tabular}

Abb. 17 IRR (Paar 3)

\begin{tabular}{|l|c|c|c|c|c|c|}
\hline \multicolumn{1}{|c|}{ IRR (Paar 3) } & \multicolumn{2}{|c|}{ W } & \multicolumn{2}{c|}{ RO } & \multicolumn{2}{c|}{ RS } \\
\hline & Kappa & $\%$ & Kappa & $\%$ & Kappa & $\%$ \\
\hline Barber *1 & .67 & 72,4 & .85 & 88,1 & .75 & 80,3 \\
\hline Körner *2 & .66 & 72,4 & .71 & 75 & .73 & 77,6 \\
\hline LU (v)*3 & .69 & 75 & .75 & 77,6 & .81 & 84,2 \\
\hline LU (o)*4 & .55 & 60,5 & .61 & 64,5 & .76 & 78,9 \\
\hline
\end{tabular}

$\mathrm{K}<0=$ schlechte Übereinstimmung $/ \mathrm{k}>.0=$ etwas Übereinstimmung $/ \mathrm{k}>.21=$ ausreichende Übereinstimmung $/ \mathrm{k}>.41=$ mittelmäßige Übereinstimmung $/ \mathrm{k}>.61=$ beachtliche Übereinstimmung $/ \mathrm{k}>.81$ = vollkommene Übereinstimmung (nach Landis und Koch 1977)

$* 1$ Barber et al. $1990 * 2$ Körner et al. $2002 * 3$ Albani et al. 2002 a,b v=vereinfacht ohne Subkategorien *4 o=original mit Subkategorien 
Fazit:

1. Alle drei IR-Paare erreichen hohe Übereinstimmungswerte. Nach Landis und Koch (1977) kann sie überwiegend als „,beachtlich“ eingestuft werden

2. Wie erwartet zeigen die ,originalen“ LU-Cluster (mit Subkategorien) die insgesamt geringsten Übereinstimmungswerte, während die „,vereinfachte“ Version sehr gute (beachtlich bis vollkommen) Übereinstimmungswerte erbringt

3. Mit den alternativen Körner-Clustern können keine höheren IR-Übereinstimmungen erzielt werden, als mit den klassischen ZBKT-Clustern

4. Die Kappa-Werte zeigen ein mit den Prozentangaben übereinstimmendes Bild. 


\subsection{Das ZBKT im Auszählverfahren}

Folgender Abschnitt setzt sich mit der zweiten allgemeinen Fragestellung der Dissertation auseinander. Gibt es ein zentrales Thema angstgestörter Patienten?

Dazu werden die ZBKT`s im AV bestimmt. Die häufigsten Cluster werden abgelesen. Die Bedeutung der Häufigkeit eines Clusters ist groß, wenngleich sie auch vorsichtig interpretiert sein will. Was in Narrativen häufig vorkommt, muss nicht zwingend der Häufigkeit im Verhalten eines Patienten entsprechen. Es bedeutet vielmehr, dass etwas eine Rolle spielt und es zu konflikthaften Auseinandersetzungen kommt.

Die Abbildungen zeigen die Häufigkeitsverteilung innerhalb der klassischen ZBKT-Cluster (Barber et al. 1990) und der reformulierten LU-Cluster (Albani et al. 2002 a,b).

\subsubsection{Klassische ZBKT-Cluster - die häufigsten W-, RO- und RS-Kategorien}

(dargestellt als prozentualer Anteil des Gesamtkollektives)

Abb.18 Häufigkeit der klassischen ZBKT-W-Cluster im Gesamtkollektiv

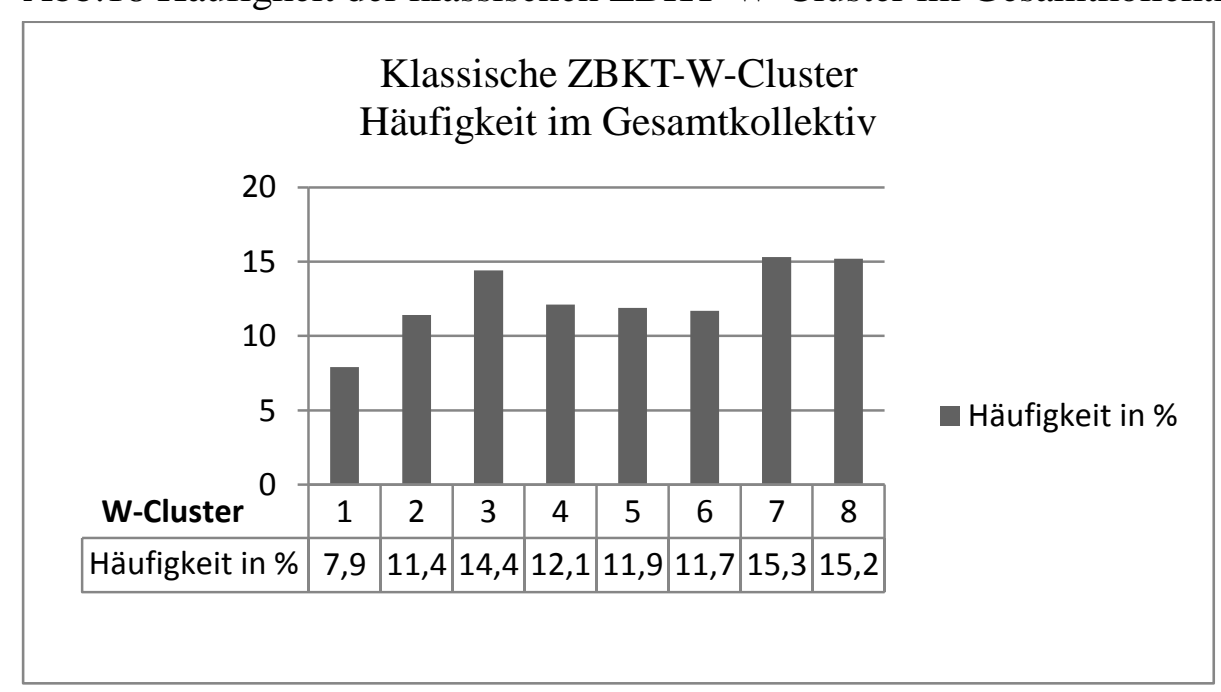

Die häufigsten Wunsch-Cluster sind 7, 8 und 3

1. Cluster 7 „Mich gut und wohl fühlen“

2. Cluster 8 „Ich möchte Erfolg, Leistung erreichen und anderen helfen“

3. Cluster 3 ,Kontrolliert und verletzt werden und keine Verantwortung haben“" 
Abb. 19 Häufigkeit der klassischen ZBKT-RO-Cluster im Gesamtkollektiv

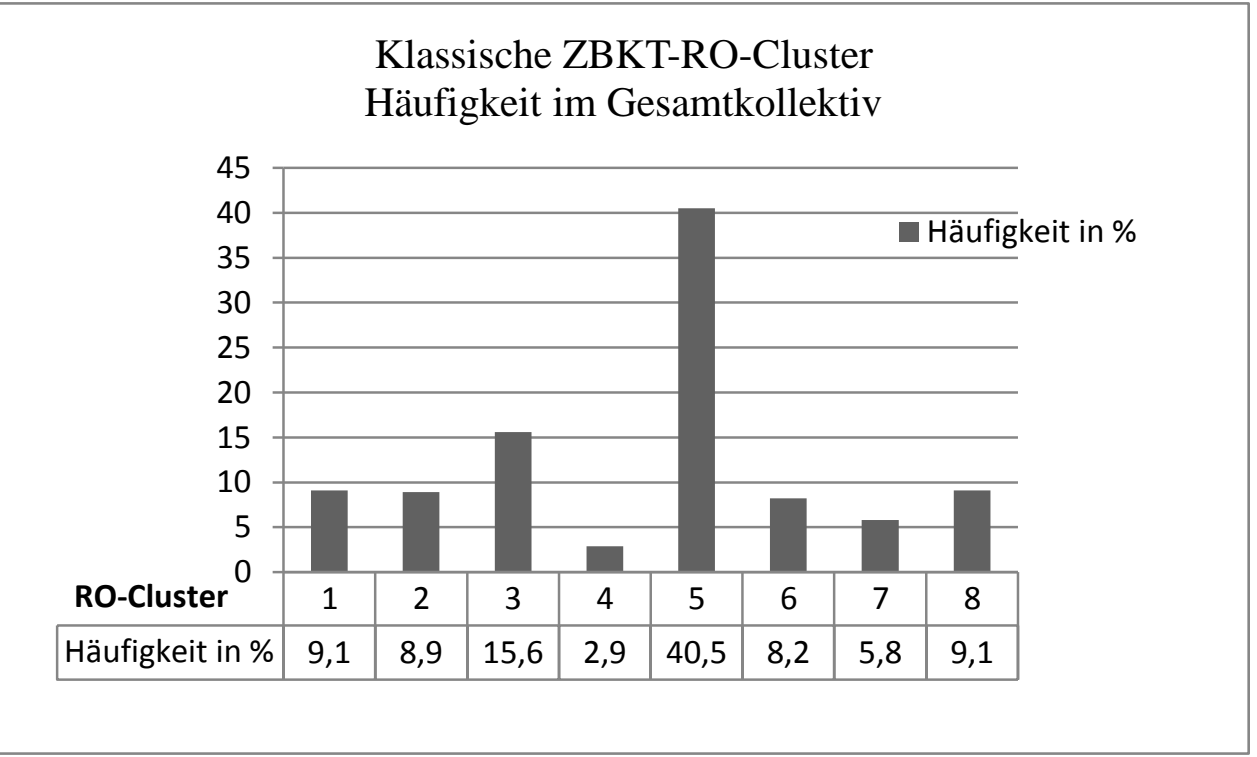

Der häufigste RO-Cluster ist Cluster 5 ,andere weisen zurück und sind gegen mich“

Abb. 20 Häufigkeit der klassischen ZBKT-RS-Cluster im Gesamtkollektiv

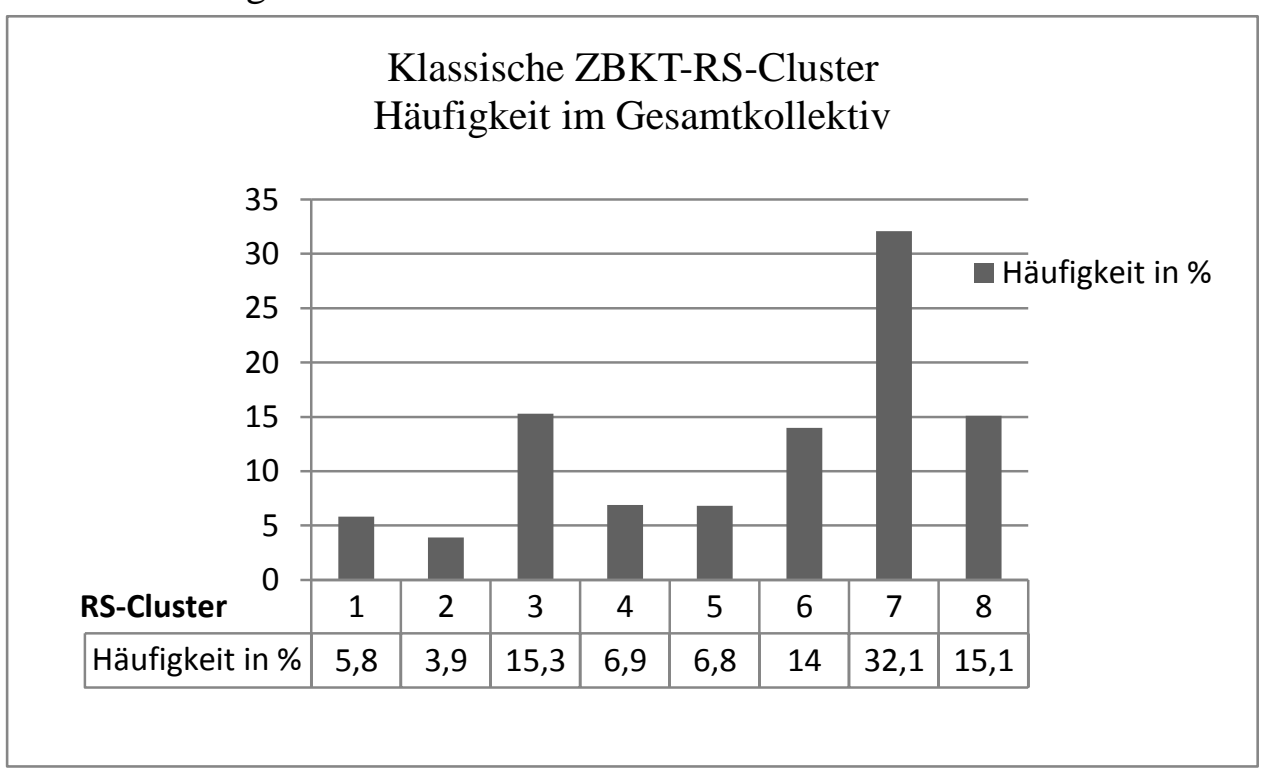

Der häufigste RS-Cluster ist RS-Cluster 7 ,ich bin enttäuscht und deprimiert“

\subsubsection{Das AV-ZBKT (klassische ZBKT-Cluster) des Gesamtkollektivs}

"Ich möchte mich gut und wohl fühlen, Erfolg und Leistung erreichen und anderen helfen, aber auch kontrolliert und verletzt werden und keine Verantwortung haben. Andere weisen mich zurück und sind gegen mich. Ich bin enttäuscht und deprimiert" 
3.2.3 LU-Cluster - die häufigsten WO-, WS-, RO- und RS-Kategorien (dargestellt als prozentualer Anteil des Gesamtkollektives)

Abb. 21 Häufigkeit der LU-WO-Cluster im Gesamtkollektiv

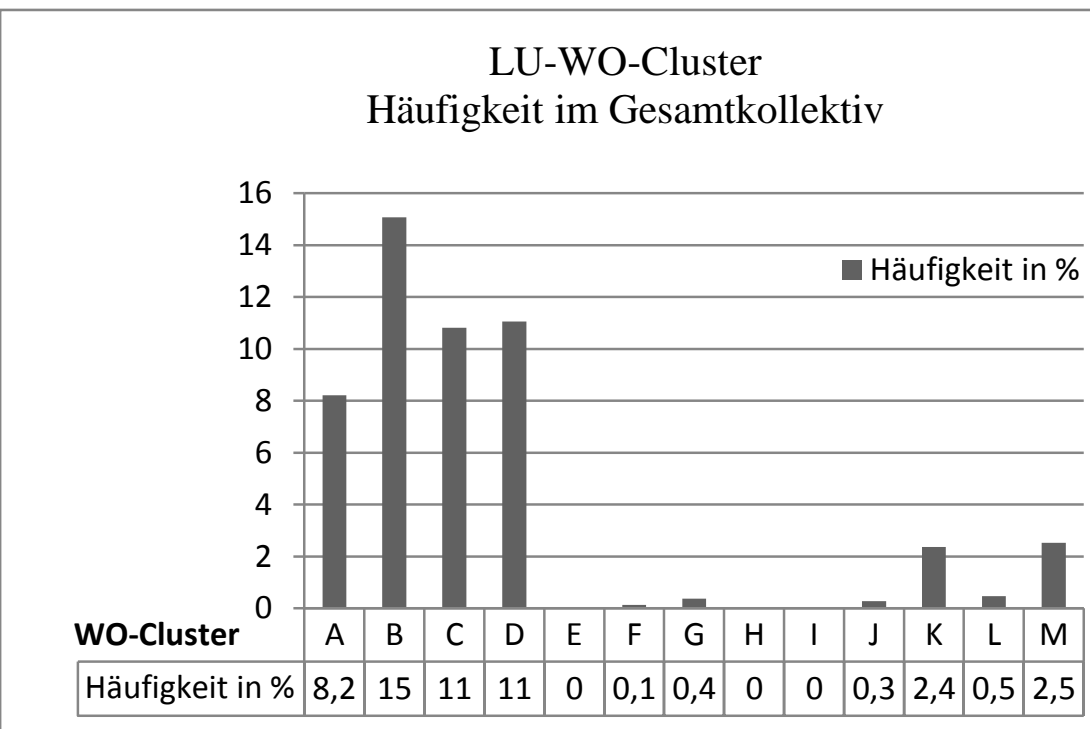

Die häufigsten WO-Cluster sind B, D, C und A

1. B ,andere sollen mich unterstützen“"

2. D ,andere sollen souverän sein“"

3. C ,andere sollen mich lieben“"

4. A ,andere sollen sich mir zuwenden“ 
Abb. 22 Häufigkeit der LU-WS-Cluster im Gesamtkollektiv

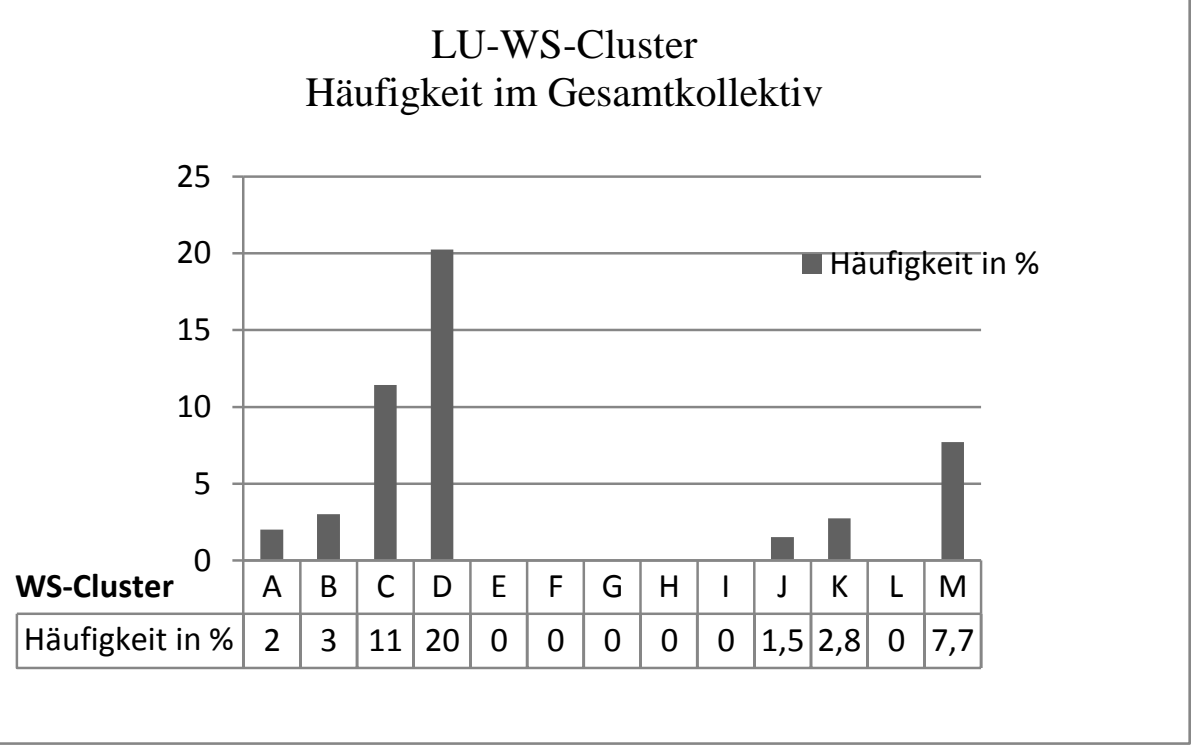

Die häufigsten WS-Cluster sind D, C und M

1. D ,ich möchte souverän sein“

2. $\mathrm{C}$,,ich möchte lieben, mich wohlfühlen“

3. $\mathrm{M}$,ich möchte mich zurückziehen“

Abb. 23 Häufigkeit der LU-RO-Cluster im Gesamtkollektiv

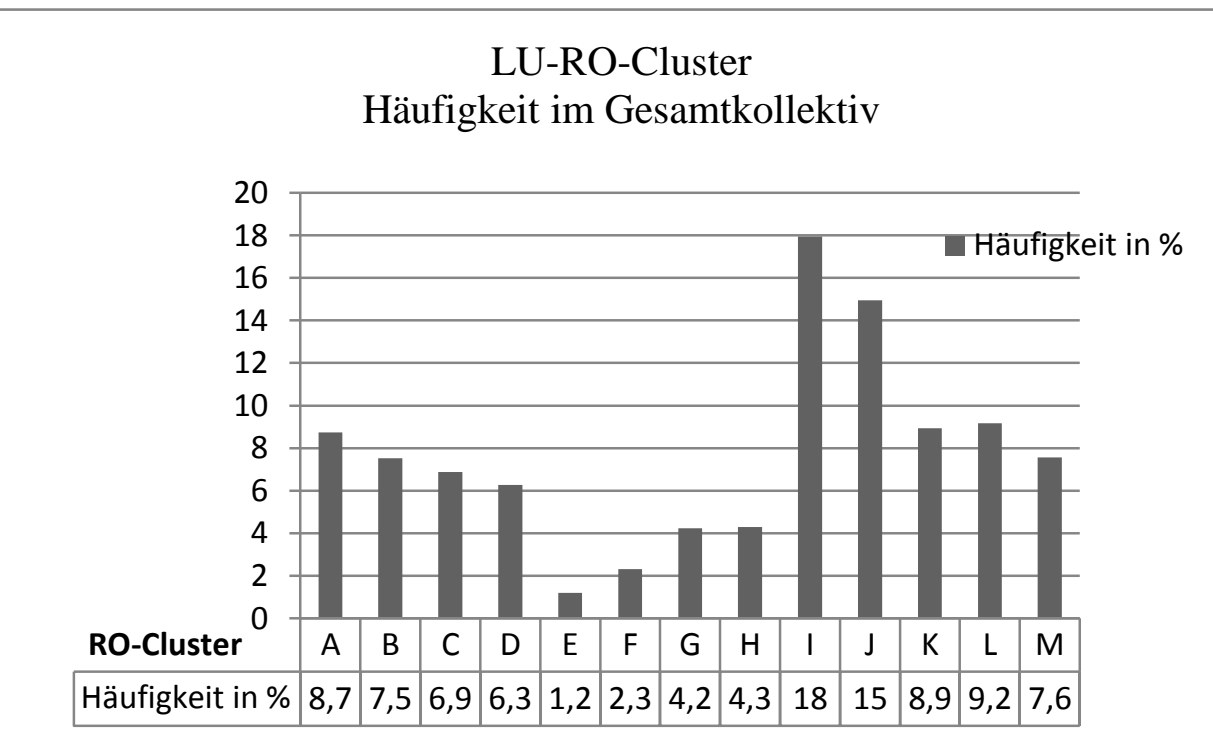

Die häufigsten RO-Cluster sind I und J

1. I ,andere sind unzuverlässig“ (18\%)

2. J , andere weisen zurück“ (15\%)

3. Alle RO-Cluster, v.a. Disharmonische, werden genannt

4. Verglichen mit den klassischen Clustern eher breite Clusterverteilung 
Abb. 24 Häufigkeit der LU-RS-Cluster im Gesamtkollektiv

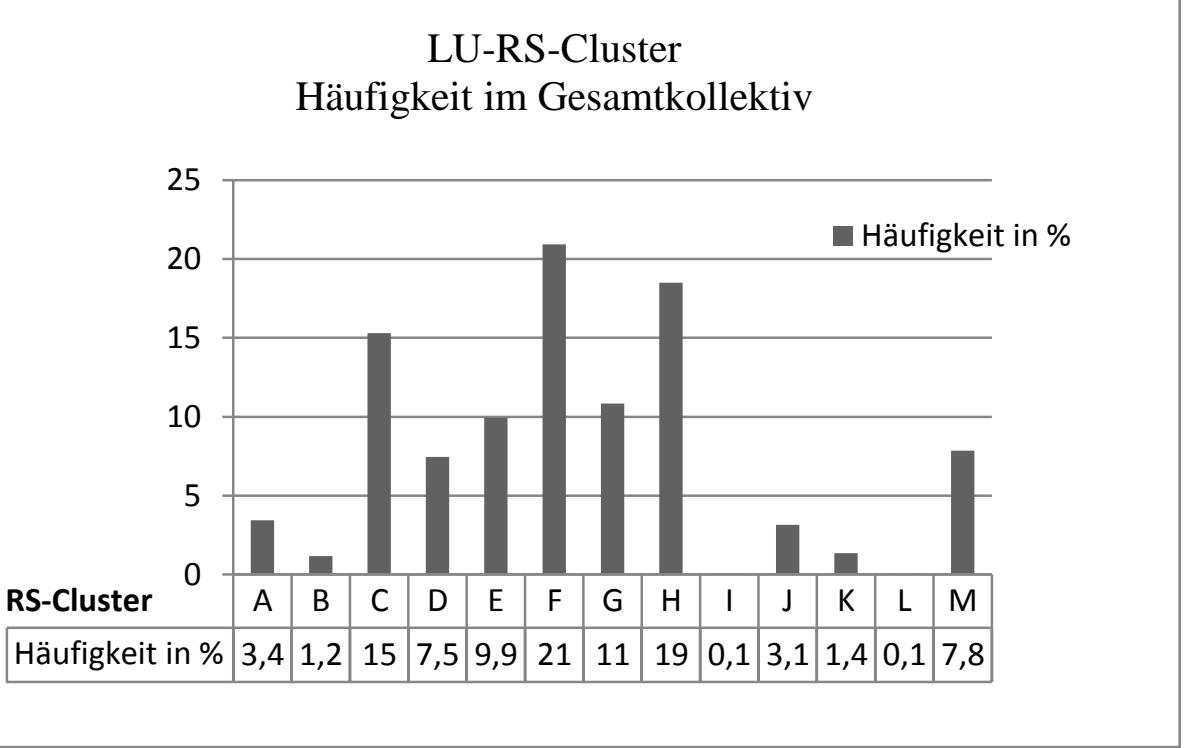

Die häufigsten RS-Cluster sind F, H und C

1. F ,unzufrieden sind, Angst haben“"

2. H , verärgert, unsympathisch sein“"

3. C ,lieben, wohlfühlen“"

4. Verglichen mit den klassischen Clustern eher breite Clusterverteilung

\subsubsection{Das AV-ZBKT (LU-Cluster) des Gesamtkollektivs}

„,Andere sollen mich unterstützen, souverän sein, mich lieben und sich mir zuwenden, ich möchte souverän sein, mich wohlfühlen und zurückziehen können. Andere sind unzuverlässig und weisen mich zurück. Ich bin unzufrieden, habe Angst und bin verärgert, fühle mich aber auch wohl" 


\subsection{Visualisierung - Erkennen häufigster Wünsche und Reaktionen im Gesamtkollektiv}

Wünsche:

Abb. 25 Visualisierung der häufigsten W im Gesamtkollektiv (klassische W-Cluster)

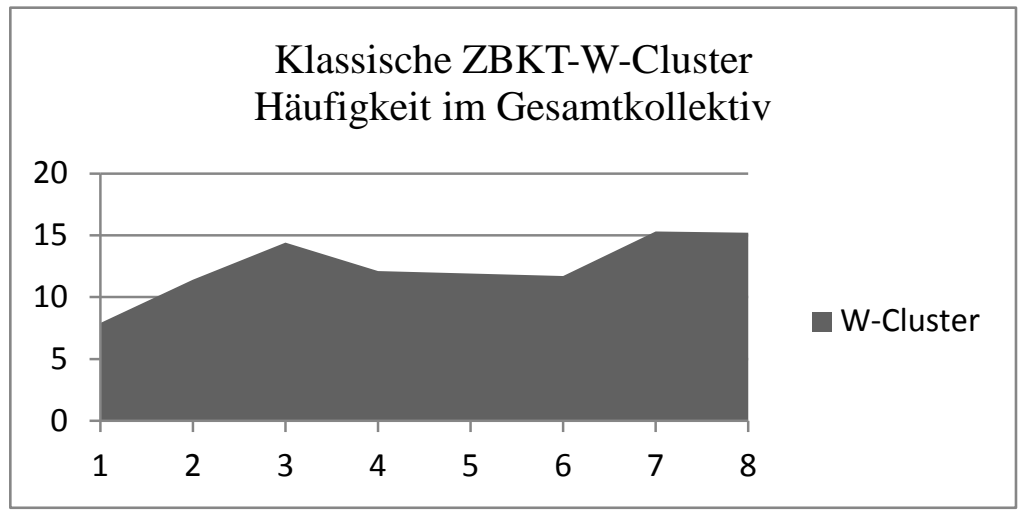

Abb. 26 Visualisierung der häufigsten W im Gesamtkollektiv (LU-WO-Cluster)

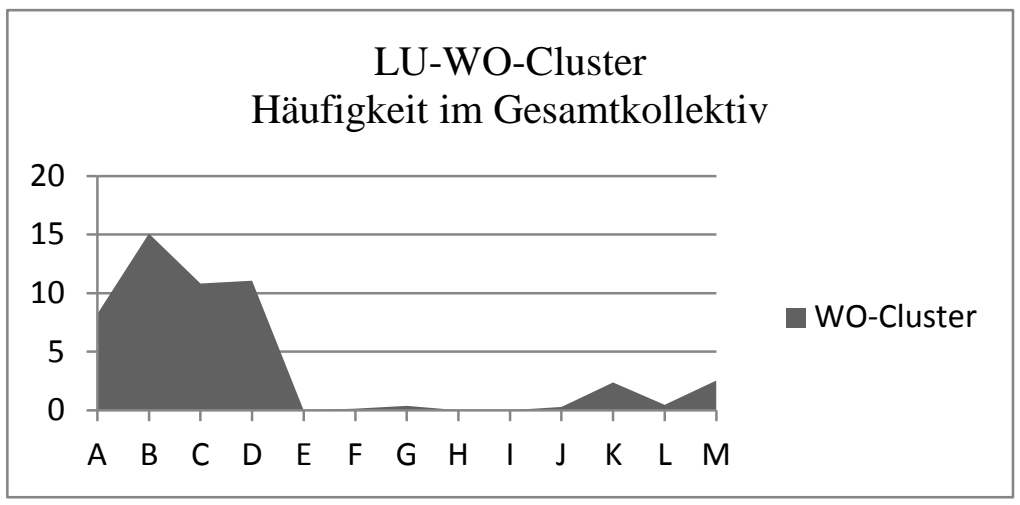

Abb. 27 Visualisierung der häufigsten W im Gesamtkollektiv (LU-WS-Cluster)

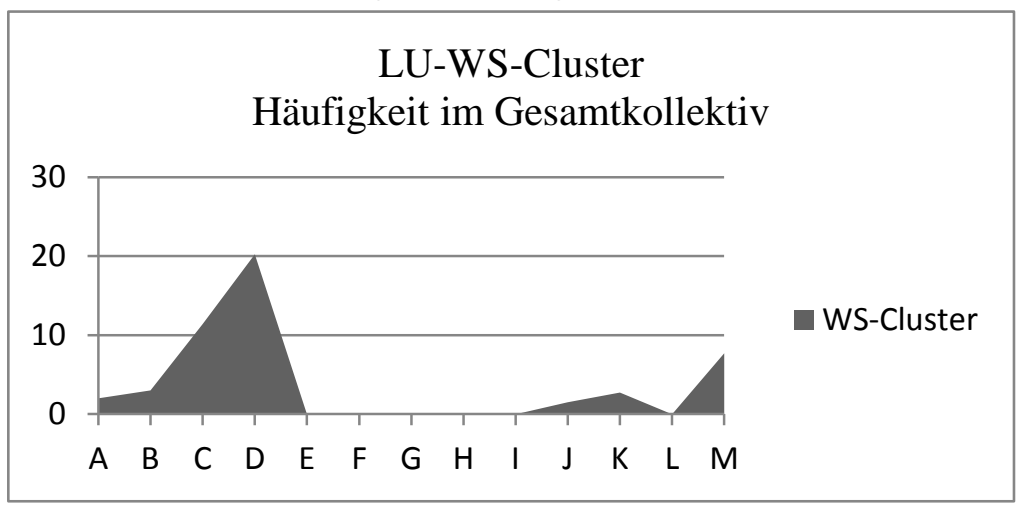


$\underline{\text { Reaktionen des Objektes: }}$

Abb. 28 Visualisierung der häufigsten RO im Gesamtkollektiv (klassische Cluster)

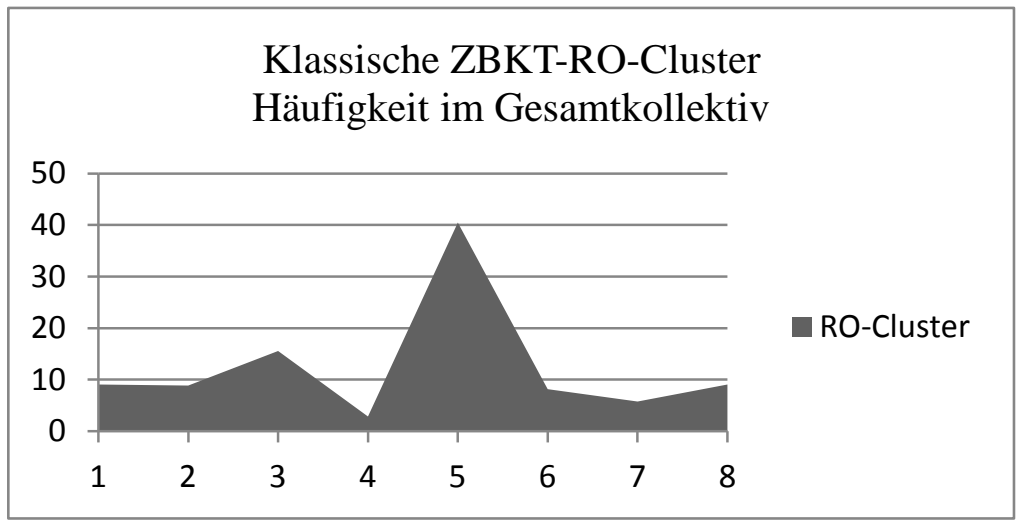

Abb. 29 Visualisierung der häufigsten RO im Gesamtkollektiv (LU-Cluster)

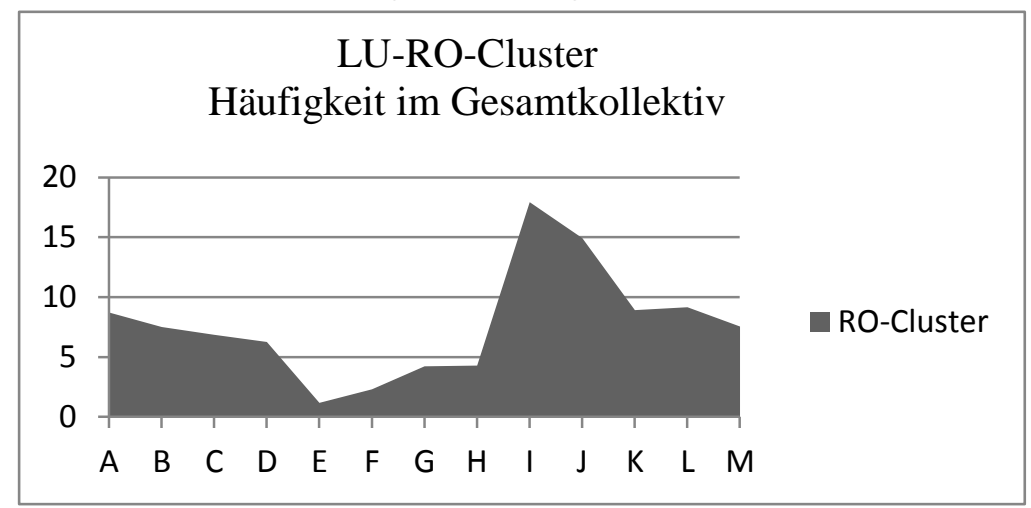


Reaktionen des Subjektes:

Abb. 30 Visualisierung der häufigsten RS im Gesamtkollektiv (klassische Cluster)

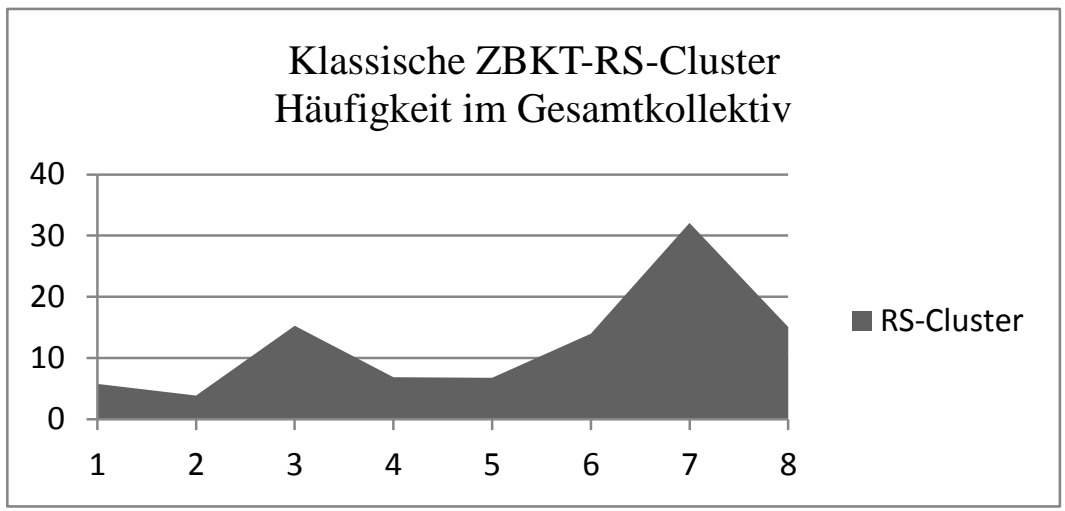

Abb. 31 Visualisierung der häufigsten RS im Gesamtkollektiv (LU-Cluster)

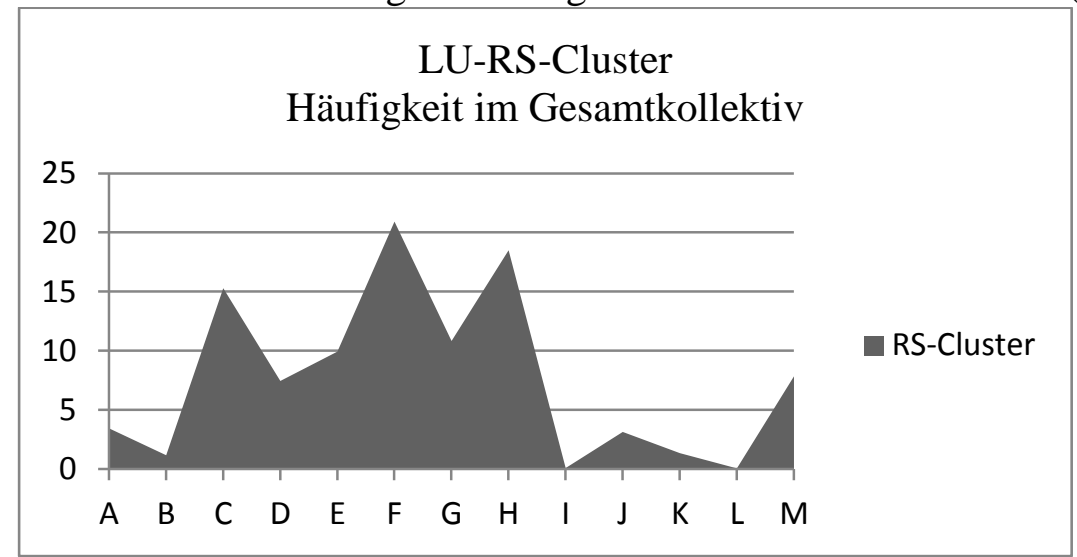




\subsection{Die Häufigkeitsverteilung der klassischen ZBKT-Cluster bei SET- und VT-Patienten}

Der folgende Abschnitt befasst sich mit der dritten allgemeinen Fragestellung. Gibt es hinsichtlich zentraler Themen Unterschiede zwischen verhaltenstherapeutisch und psychodynamisch-tiefenpsychologisch behandelten Patienten? Welche Rolle spielt dabei möglicherweise die Behandlungsform?

Um diese Frage zu beantworten, wurde das Gesamtkollektiv $(\mathrm{N}=45)$ in eine SET- und eine VT-Gruppe unterteilt und die AV-ZBKT`s separat für diese Gruppen bestimmt. Erneut wurden sowohl die klassischen ZBKT-Cluster, als auch die reformulierten LU-Cluster verwendet.

\subsubsection{Die häufigsten W-, RO- und RS-Kategorien der SET- und VT-Patienten}

Abb. 32 Häufigkeit der klassischen ZBKT-W-Cluster bei SET- und VT-Patienten

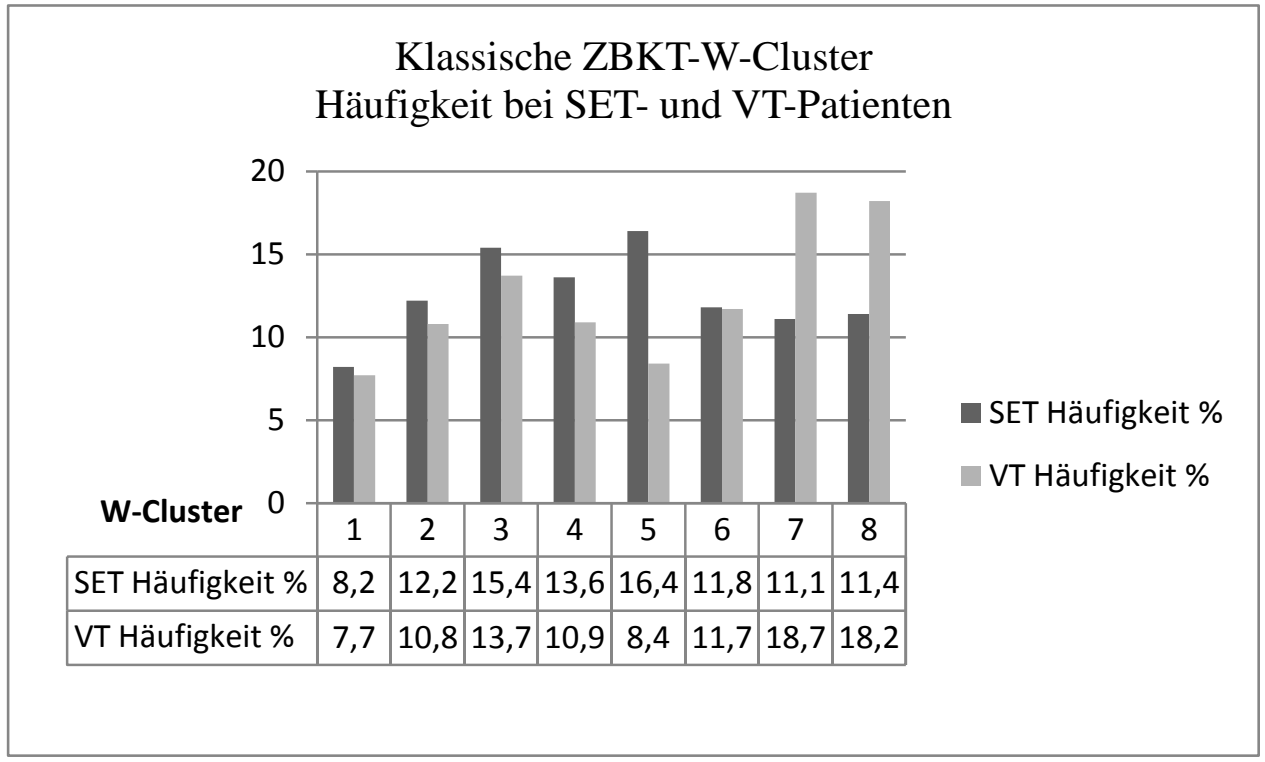

Die häufigsten Wunsch-Cluster der SET-Patienten sind 5, 3 und 4

1. Cluster 5 ,,anderen nahe sein, annehmen“"

2. Cluster 3 ,kontrolliert und verletzt werden, keine Verantwortung haben“

3. Cluster 4 ,Abstand haben, Auseinandersetzung vermeiden“

Die häufigsten Wunsch-Cluster der VT-Patienten sind 7 und 8

1. Cluster 7 ,mich gut und wohl fühlen“

2. Cluster 8 ,Erfolg und Leistung erreichen und anderen helfen“ 
Abb. 33 Häufigkeit der klassischen ZBKT-RO-Cluster bei SET- und VT-Patienten

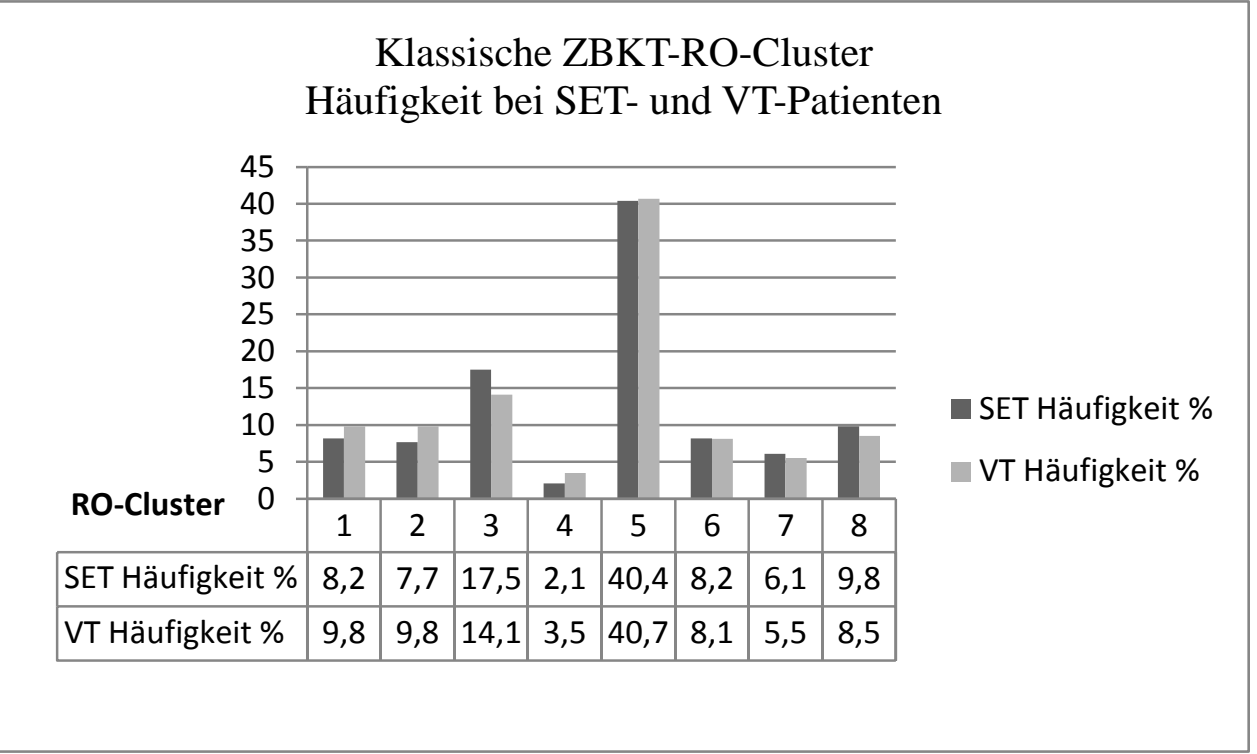

Der häufigste RO-Cluster der SET- und VT-Patienten ist Cluster 5 ,andere weisen mich zurück und sind gegen mich“"

Abb. 34 Häufigkeit der klassischen ZBKT-RS-Cluster bei SET- und VT-Patienten

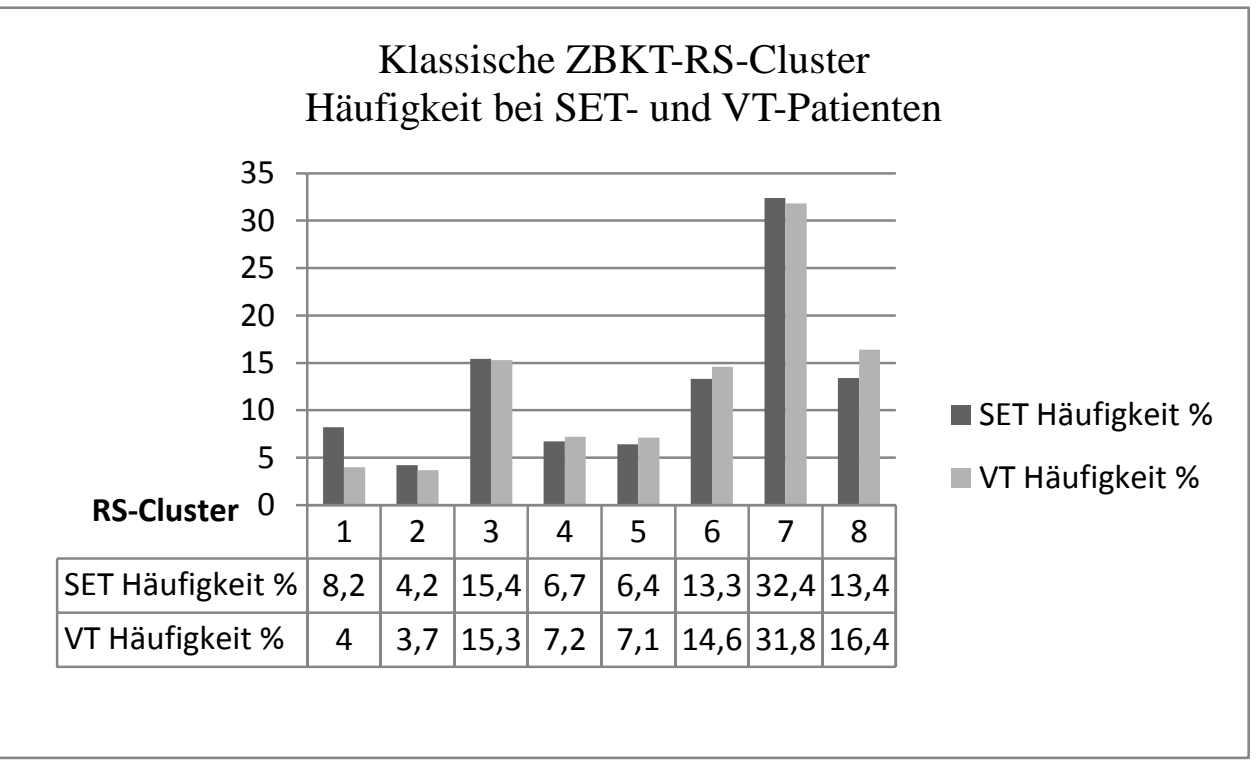

Der häufigste RS-Cluster der SET- und VT-Patienten ist Cluster 7 ,ich bin enttäuscht und deprimiert"، 


\subsubsection{Das ZBKT der SET-Patienten}

„Ich möchte anderen nahe sein, andere annehmen. Ich möchte keine Verantwortung, sondern Abstand haben und Auseinandersetzungen vermeiden. Andere weisen mich zurück und sind gegen mich. Ich bin enttäuscht und deprimiert"

\subsubsection{Das ZBKT der VT-Patienten}

„Ich möchte mich gut und wohl fühlen und Erfolg und Leistung erreichen. Ich möchte anderen helfen. Andere weisen mich zurück und sind gegen mich. Ich bin enttäuscht und deprimiert" 


\subsection{Die Häufigkeitsverteilung der LU-Cluster bei SET- und VT-Patienten}

\section{$\underline{\text { 3.5.1 Die häufigsten W-, RO- und RS-Kategorien der SET- und VT-Patienten }}$}

Abb. 35 Häufigkeit der LU-WO-Cluster bei SET- und VT-Patienten

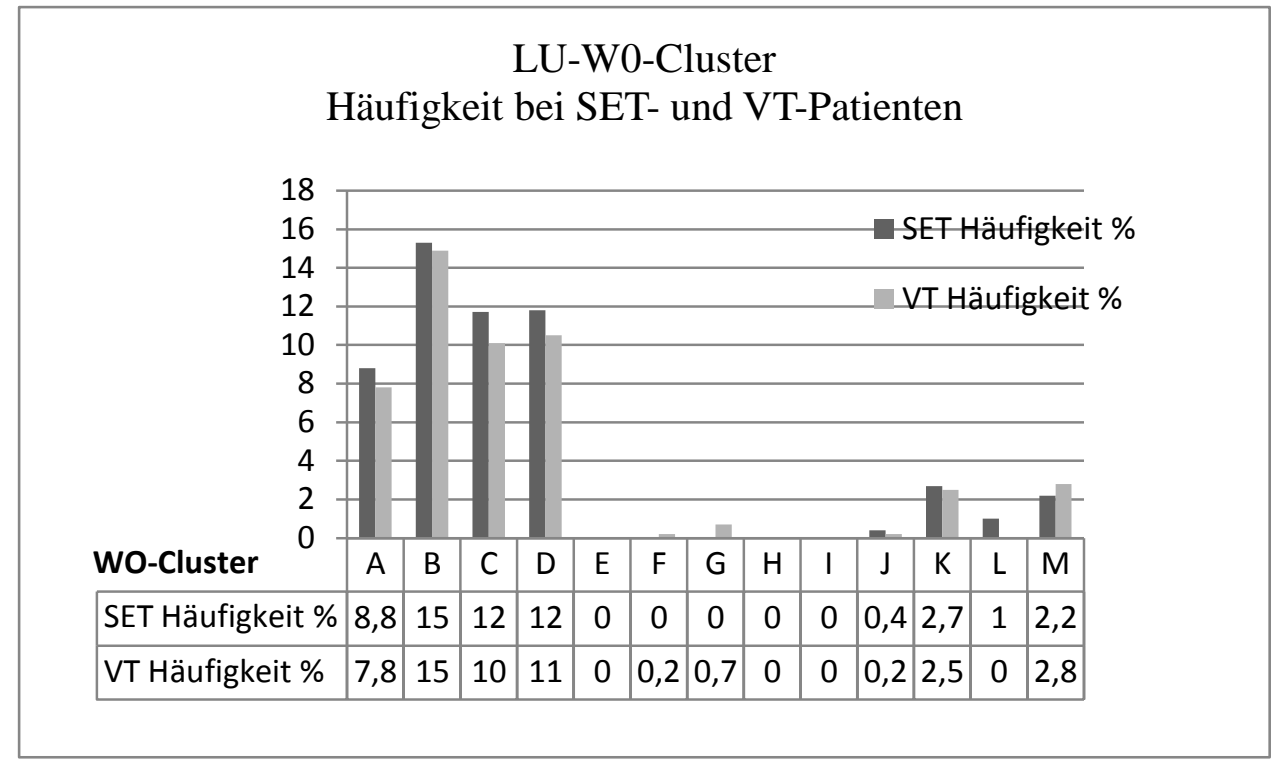

Die häufigsten WO-Cluster der SET-Patienten sind B, C, D und A

1. B ,andere sollen mich unterstützen“

2. C ,andere sollen mich lieben, wohlfühlen“"

3. D ,andere sollen souverän sein“

4. A ,andere sollen sich mir zuwenden“

Die häufigsten WO-Cluster der VT-Patienten sind B, D, C und A

1. B ,andere sollen mich unterstützen“

2. D ,andere sollen souverän sein“"

3. C ,andere sollen mich lieben, wohlfühlen“"

4. A, ,andere sollen sich mir zuwenden“ 
Abb. 36 Häufigkeit der LU-WS-Cluster bei SET- und VT-Patienten

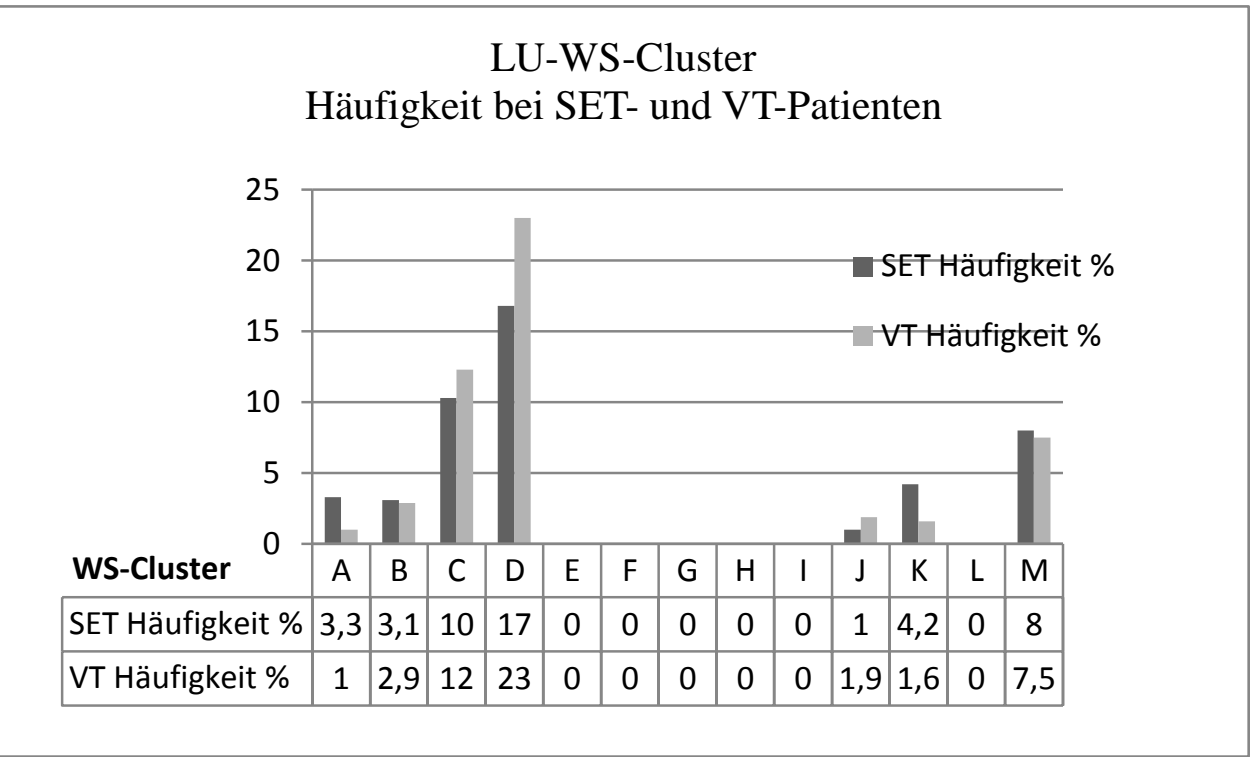

Die häufigsten WS-Cluster der SET- und VT-Patienten sind D, C und M

1. D ,ich möchte souverän sein“

2. C ,ich möchte lieben, mich wohlfühlen“"

3. $\mathrm{M}$,ich möchte mich zurückziehen“

Abb. 37 Häufigkeit der LU-RO-Cluster bei SET- und VT-Patienten

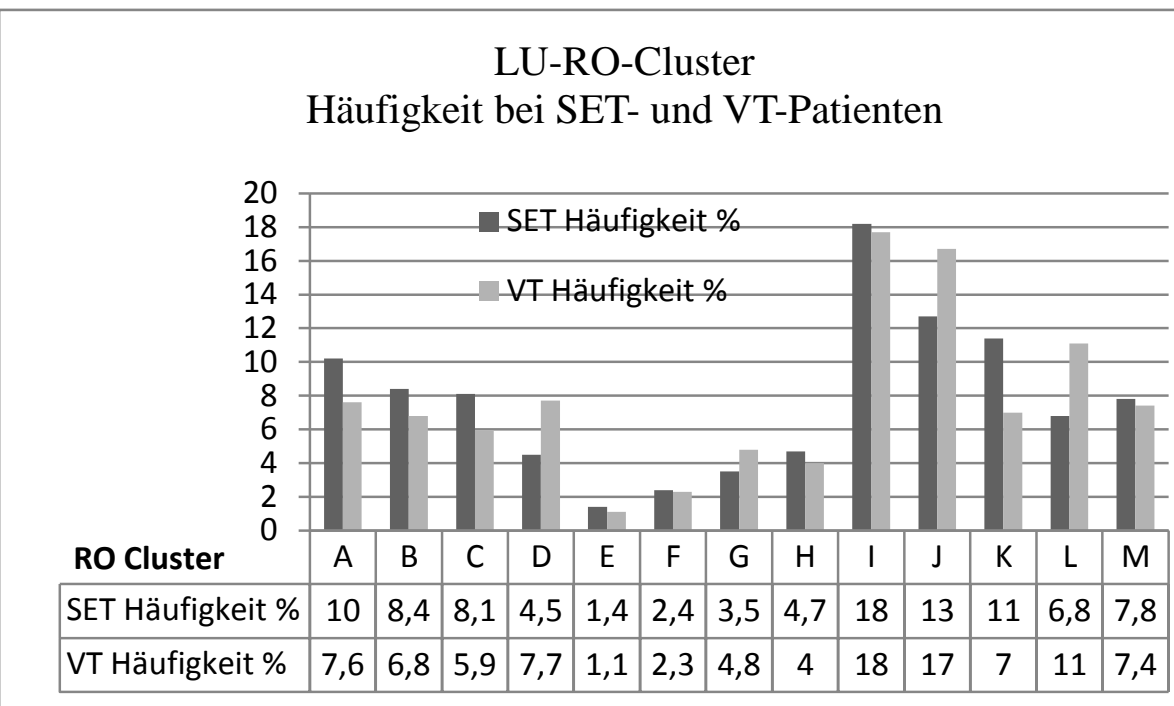

Die häufigsten RO-Cluster der SET-Patienten sind I, J, K und A

1. I , andere sind unzuverlässig“"

2. J ,,andere weisen zurück“"

3. $\mathrm{K}$,,andere dominieren“"

4. A ,andere wenden sich $\mathrm{zu}^{\circ}$ 
Die häufigsten RO-Cluster der VT-Patienten sind I, J und L

1. I ,,andere sind unzuverlässig““

2. $\mathrm{J}$,,andere weisen zurück“

3. L ,andere ärgern, greifen an“

Abb. 38 Häufigkeit der LU-RS-Cluster bei SET- und VT-Patienten

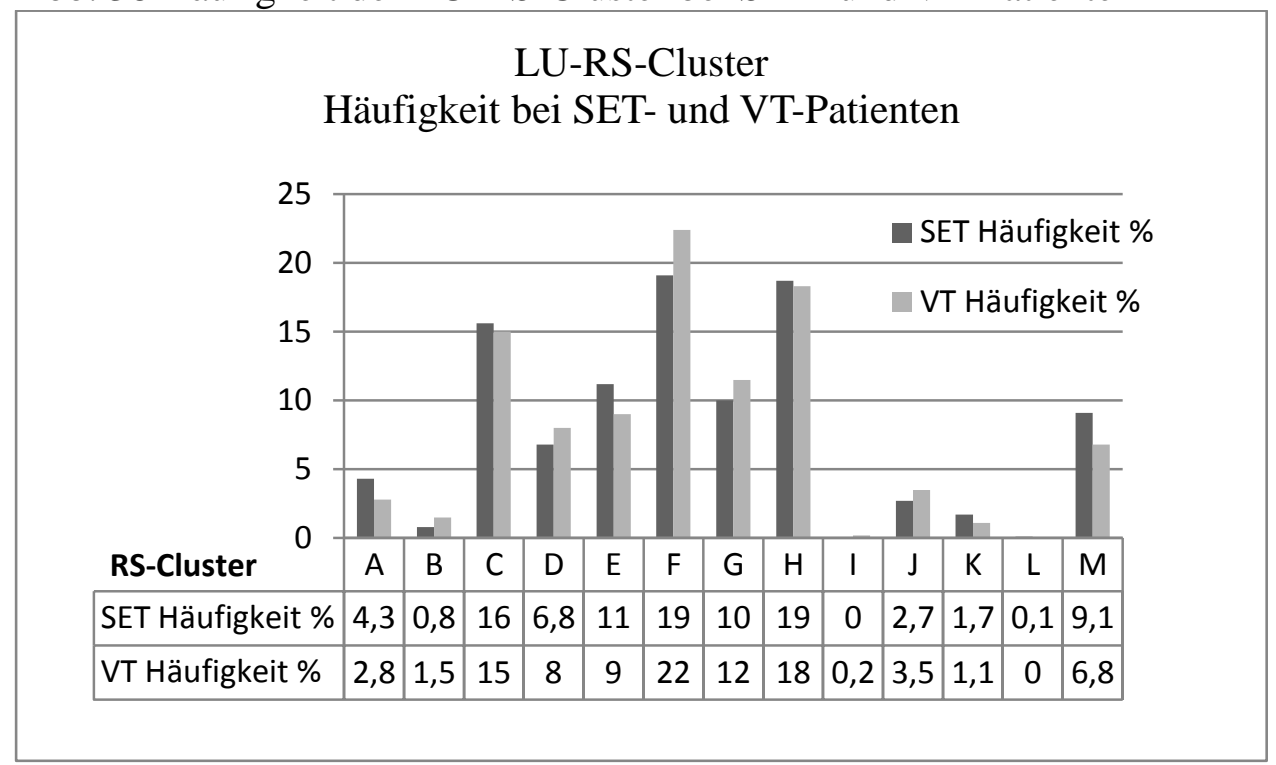

Die häufigsten RS-Cluster SET- und VT-Patienten sind F, H und C

1. F ,unzufrieden sein, Angst haben“

2. H ,verärgert, unsympathisch sein“

3. C ,lieben, wohlfühlen“

\subsubsection{Das ZBKT der SET-Patienten}

„Andere sollen mich unterstützen, mich lieben, souverän sein und sich mir zuwenden. Ich möchte souverän sein. Andere sind unzuverlässig. Ich bin unzufrieden, habe Angst und bin verärgert"

\subsubsection{Das ZBKT der VT-Patienten}

„,Andere sollen mich unterstützen, mich lieben, souverän sein und sich zuwenden. Ich möchte souverän sein. Andere sind unzuverlässig, weisen mich zurück. Ich bin unzufrieden, habe Angst und bin verärgert" 


\subsection{Die Veränderungen der Häufigkeitsverteilung klassischer ZBKT- Cluster im Therapieverlauf}

Der folgende Abschnitt befasst sich mit der vierten allgemeinen Fragestellung. Verändern sich zentrale Themen während des Therapieverlaufes? Wenn ja, gibt es Unterschiede zwischen VT- und SET-Patienten? Um diese Frage angemessen zu beantworten, wurden die ZBKT`s an zwei Therapiezeitpunkten gemessen und miteinander verglichen. Zunächst folgt die Darstellung der Clusterhäufigkeit der SET- und VT-Patienten zu verschiedenen Zeitpunkten. Erneut wurden die klassischen ZBKT-Cluster und die reformulierten LU-Cluster verwendet.

\subsubsection{Die Veränderungen bei SET-Patienten}

(Gemessen wurde die prozentuale Häufigkeit eines Clusters zu zwei Therapiezeitpunkten (T1= 3.+5.Therapiestunde, $\mathrm{T} 2=17 .+18$. Therapiestunde))

Abb. 39 Veränderung der Häufigkeit klassischer ZBKT-W-Cluster von SET-Patienten

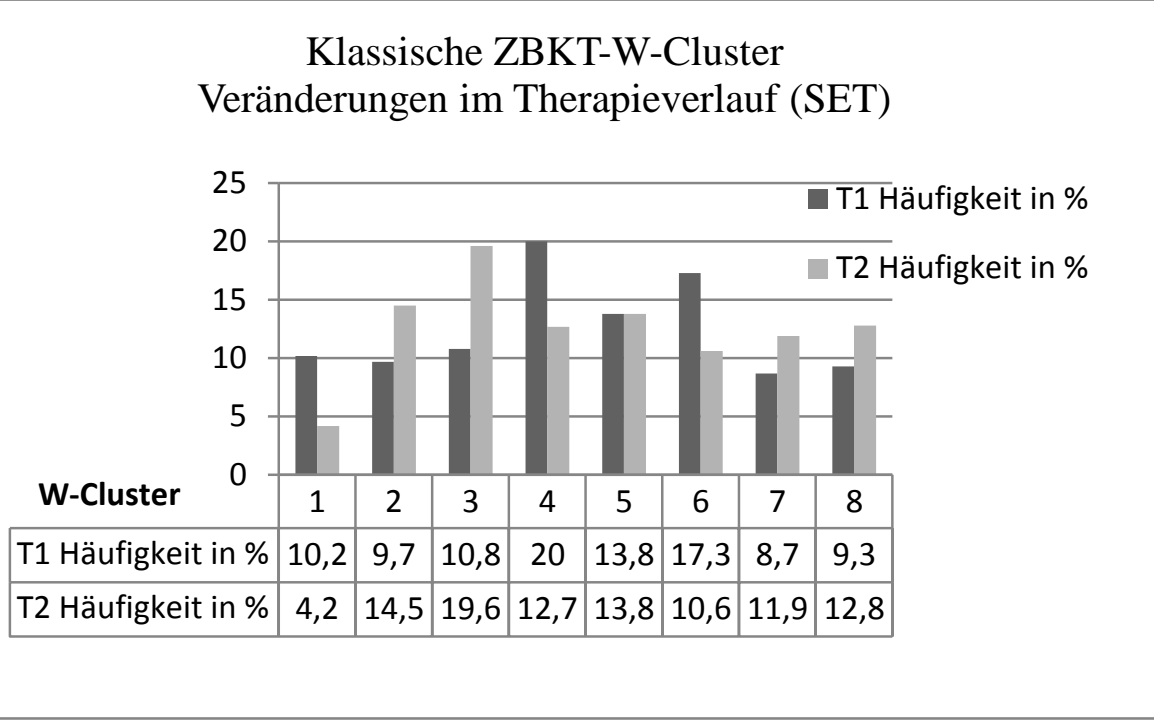

Abb. 40 Visualisierung der Veränderung des Wunsch-Clusters

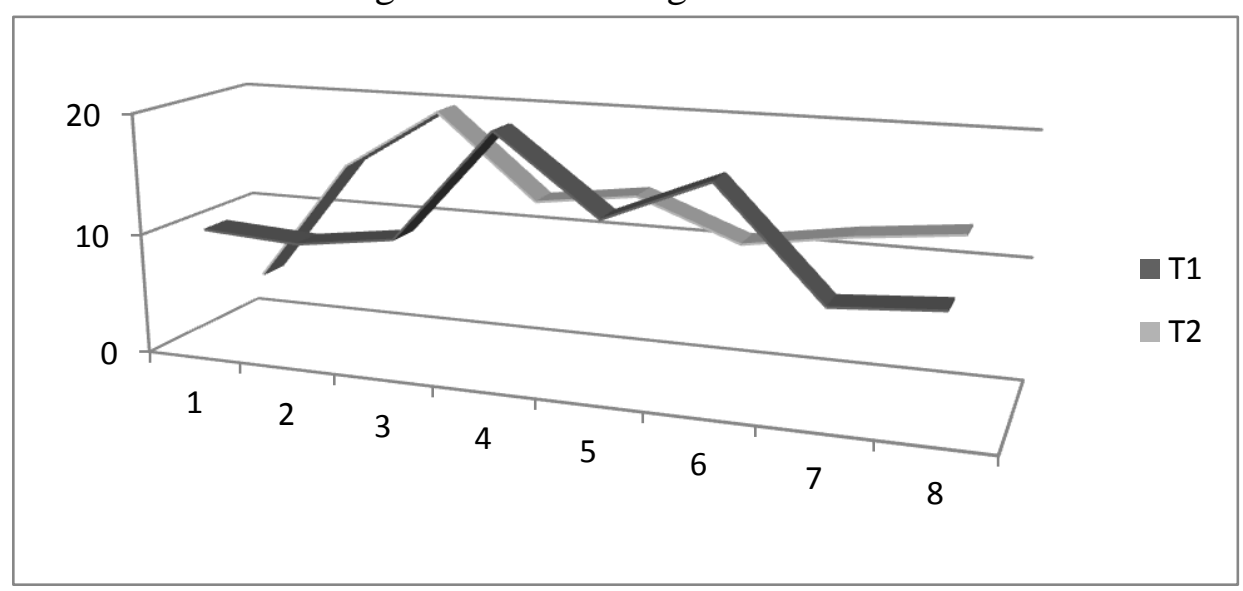


Zum Zeitpunkt $\underline{\text { T1 }}$ sind die Wunsch-Cluster 4, 6 und 5 am häufigsten

1. Cluster 4 „Abstand haben und Auseinandersetzung vermeiden“"

2. Cluster 6 "Geliebt und verstanden werden“"

3 . Cluster 5 ,anderen nahe sein und annehmen“

Zum Zeitpunkt T2 sind die Wunsch-Cluster 3, 2 und 5 am häufigsten

1. Cluster 3 „Kontrolliert und verletzt werden und keine Verantwortung haben“

2. Cluster 2 „Mich anderen widersetzen, andere verletzten und kontrollieren“

3. Cluster 5 ,anderen nahe sein und annehmen“

Stärkste Zunahme der Nennungshäufigkeit während der Therapie

1. Cluster 2 ,mich anderen widersetzen und andere verletzen und kontrollieren“

2. Cluster 3 ,Kontrolliert und verletzt werden keine Verantwortung haben“

Stärkste Abnahme der Nennungshäufigkeit während der Therapie

1. Cluster 4 ,Abstand haben und Auseinandersetzung vermeiden“

2. Cluster 1 ,mich behaupten und unabhängig sein“"

3. Cluster 6 ,geliebt und verstanden werden“"

Abb. 41 Veränderung der Häufigkeit klassischer ZBKT-RO-Cluster von SET-Patienten

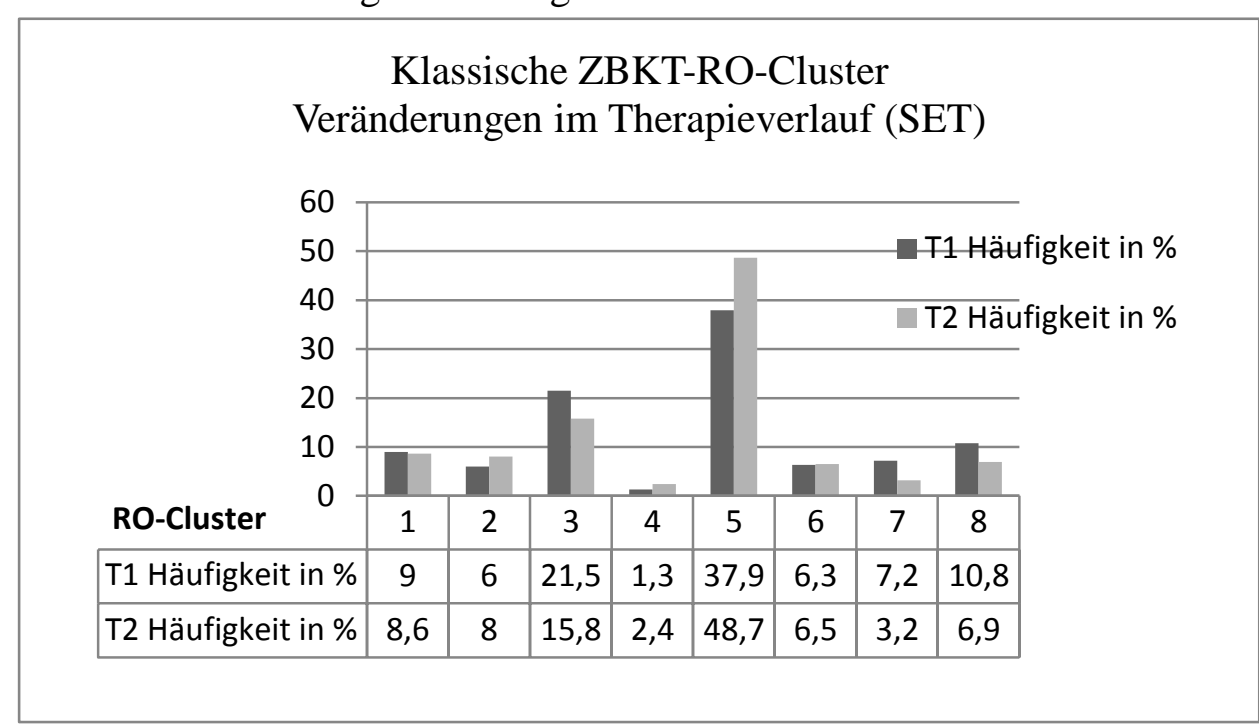

Zum Zeitpunkt T1 und T2 sind die RO-Cluster 5 und 3 am häufigsten

1. Cluster 5 ,,andere weisen zurück und sind gegen mich“ $^{\text {“ }}$

2. Cluster 3 ,andere sind bestürzt, aufgeregt und ärgerlich“

3. Stärkste Zunahme der Nennungshäufigkeit: Cluster 5

4. Stärkste Abnahme der Nennungshäufigkeit: Cluster 3 
Abb. 42 Veränderung der Häufigkeit klassischer ZBKT-RS-Cluster von SET-Patienten

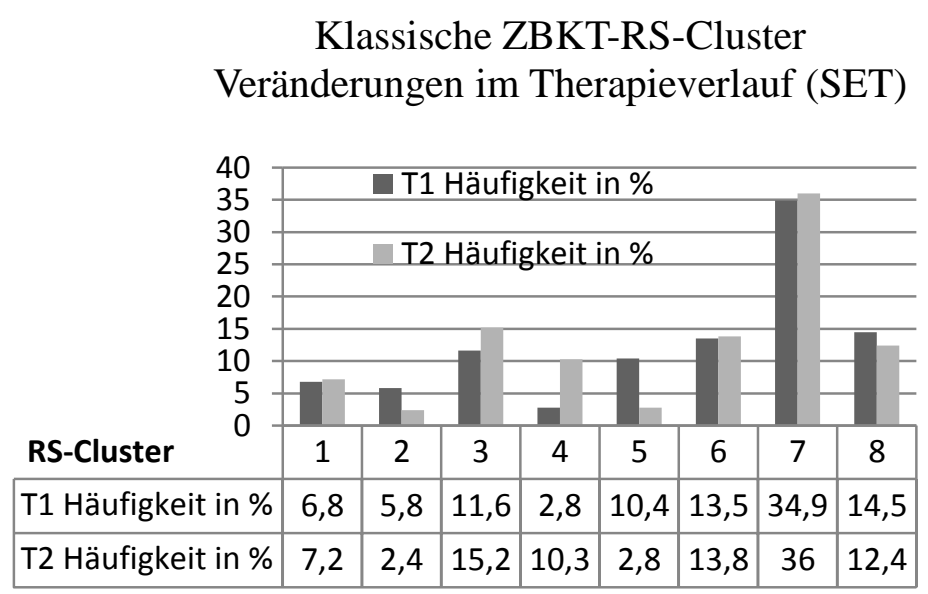

Abb. 43 Visualisierung der Veränderung des RS-Clusters

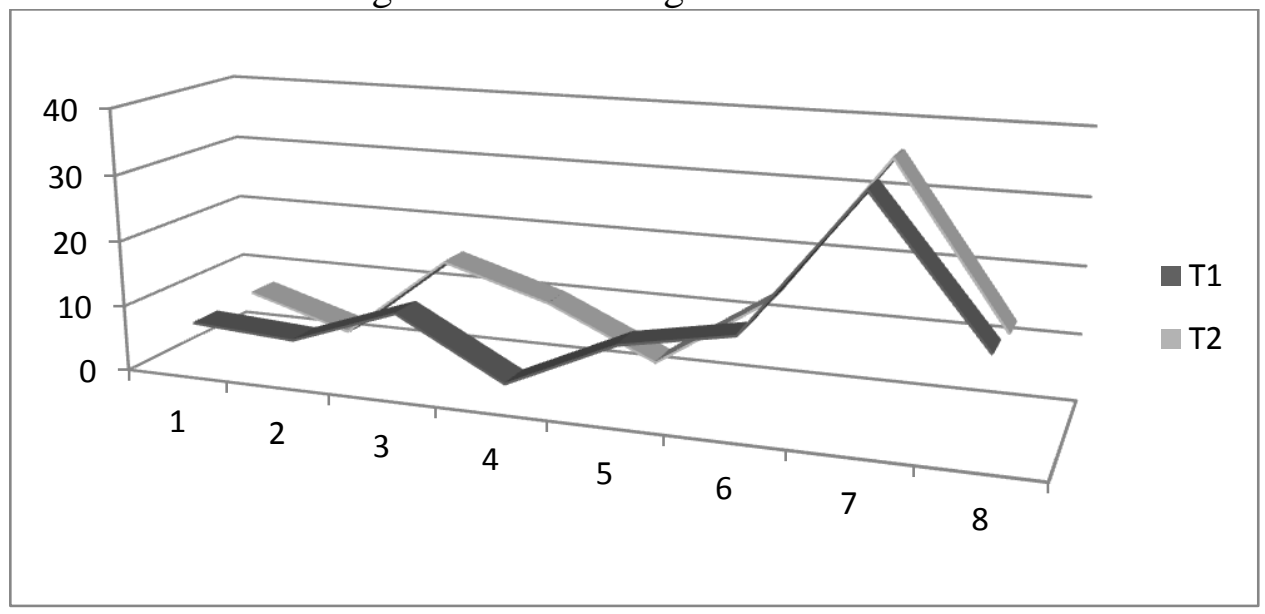

Zum Zeitpunkt T1 und T2 ist RS-Cluster 7 am häufigsten

1. Cluster 7 ,,bin enttäuscht und deprimiert“

2. Stärkste Zunahme der Nennungshäufigkeit: Cluster 4 ,,ich widersetze mich und verletze andere“ und Cluster 3 ,fühle mich respektiert und akzeptiert“

3. Stärkste Abnahme der Nennungshäufigkeit: Cluster 5 ,ich habe Selbstkontrolle und Selbstvertrauen“ und Cluster 2 ,,bin unempfänglich, unaufgeschlossen

\subsubsection{Das ZBKT der SET-Patienten zu verschiedenen Zeitpunkten (T1/T2)}

T1: „Ich möchte Abstand haben und Auseinandersetzungen vermeiden, geliebt und verstanden werden. Die anderen weisen mich zurück und sind gegen mich. Ich bin enttäuscht und deprimiert"

T2: „Ich möchte kontrolliert und verletzt werden und keine Verantwortung haben. Ich möchte mich anderen widersetzen, andere verletzen und kontrollieren. Die anderen weisen mich zurück, sind gegen mich. Ich bin enttäuscht und deprimiert “ 


\section{$\underline{\text { 3.6.3 Die Veränderungen bei VT-Patienten }}$}

(Gemessen wurde die prozentuale Häufigkeit eines Clusters zu zwei Therapiezeitpunkten (T1= 3.+5.Therapiestunde, $\mathrm{T} 2=17 .+18$. Therapiestunde))

Abb. 44 Veränderung der Häufigkeit klassischer ZBKT-W-Cluster von VT-Patienten

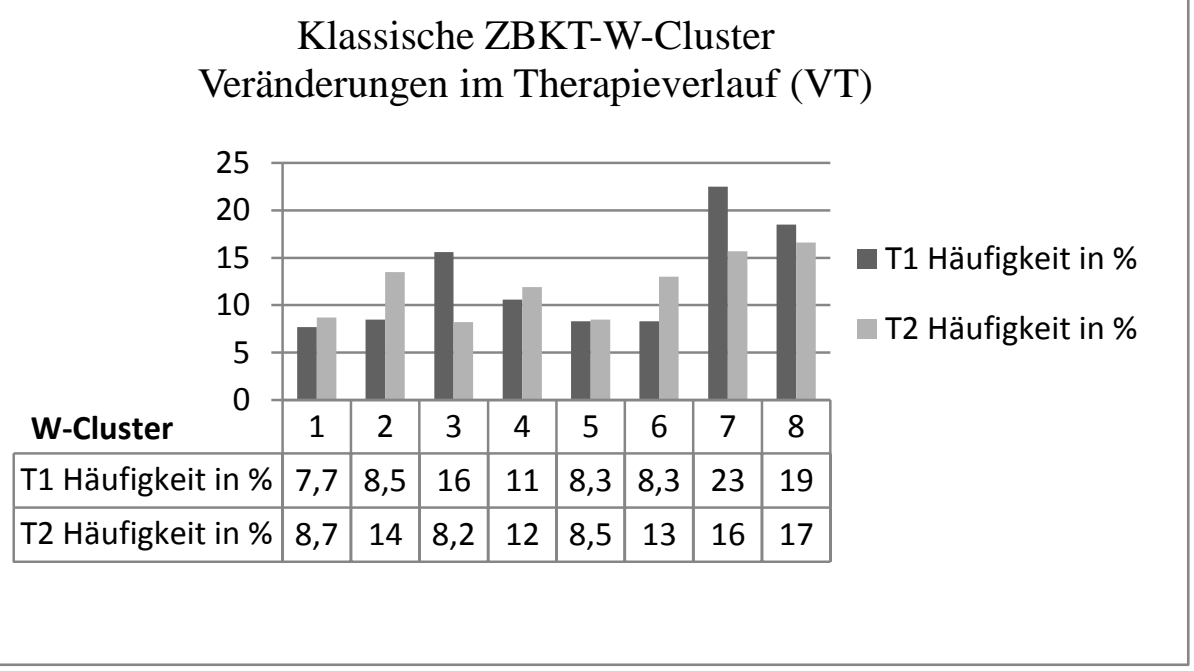

Abb. 45 Visualisierung der Veränderung des W-Clusters

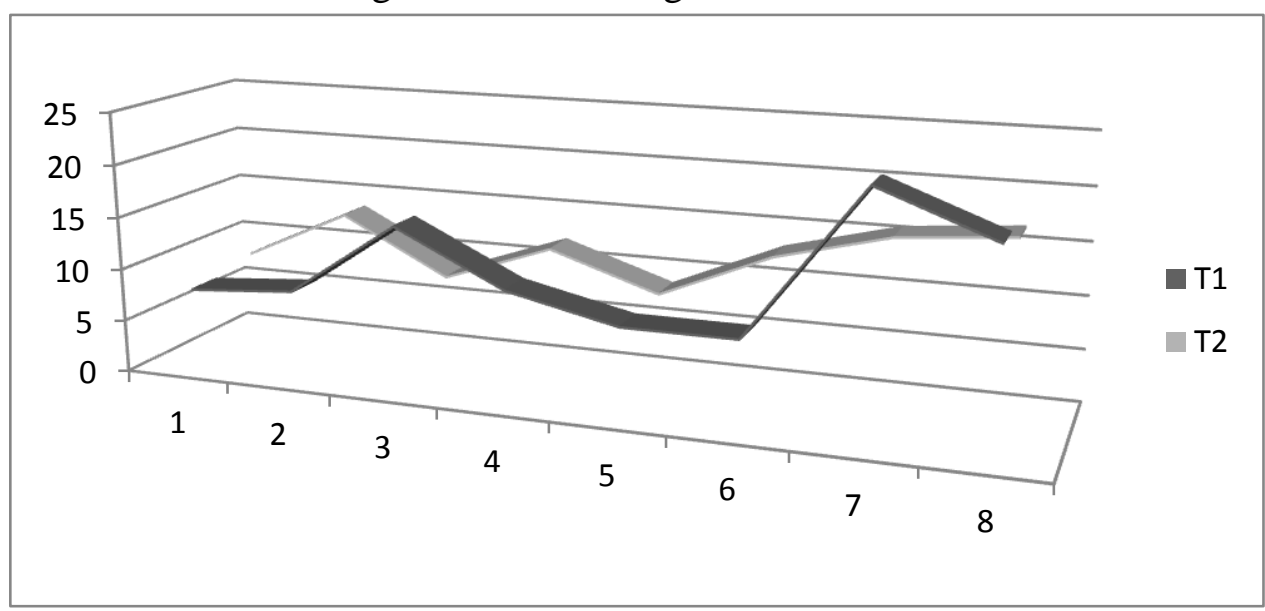

Zum Zeitpunkt T1 sind die Wunsch-Cluster 7, 8 und 3 am häufigsten

1. Cluster 7 ,mich gut und wohl fühlen“

2. Cluster 8 ,Erfolg und Leistung erreichen und anderen helfen“

3. Cluster 3 ,kontrolliert und verletzt werden und keine Verantwortung haben“

Zum Zeitpunkt T2 sind die Wunsch-Cluster 8, 7, 2 und 6 am häufigsten

1. Cluster 8 „Erfolg und Leistung erreichen und anderen helfen“"

2. Cluster 7 „mich gut und wohl fühlen“

3. Cluster 2 ,mich anderen widersetzen, andere verletzen und kontrollieren“

4. Cluster 6 ,geliebt und verstanden werden“"

5. Zum Zeitpunkt T2 zeigt sich eine breitere Verteilung der Cluster ohne deutlicheres Herausstechen eines oder mehrerer Cluster 
Stärkste Zunahme der Nennungshäufigkeit während der Therapie

1. Cluster 2 ,mich anderen widersetzen, andere verletzen und kontrollieren“ und

2. Cluster 6 ,geliebt und verstanden werden“

Stärkste Abnahme der Nennungshäufigkeit während der Therapie

1. Cluster 7 ,mich gut und wohl fühlen“ und

2. Cluster 3 ,kontrolliert und verletzt werden und keine Verantwortung haben“

Abb. 46 Veränderung der Häufigkeit klassischer ZBKT-RO-Cluster von VT-Patienten

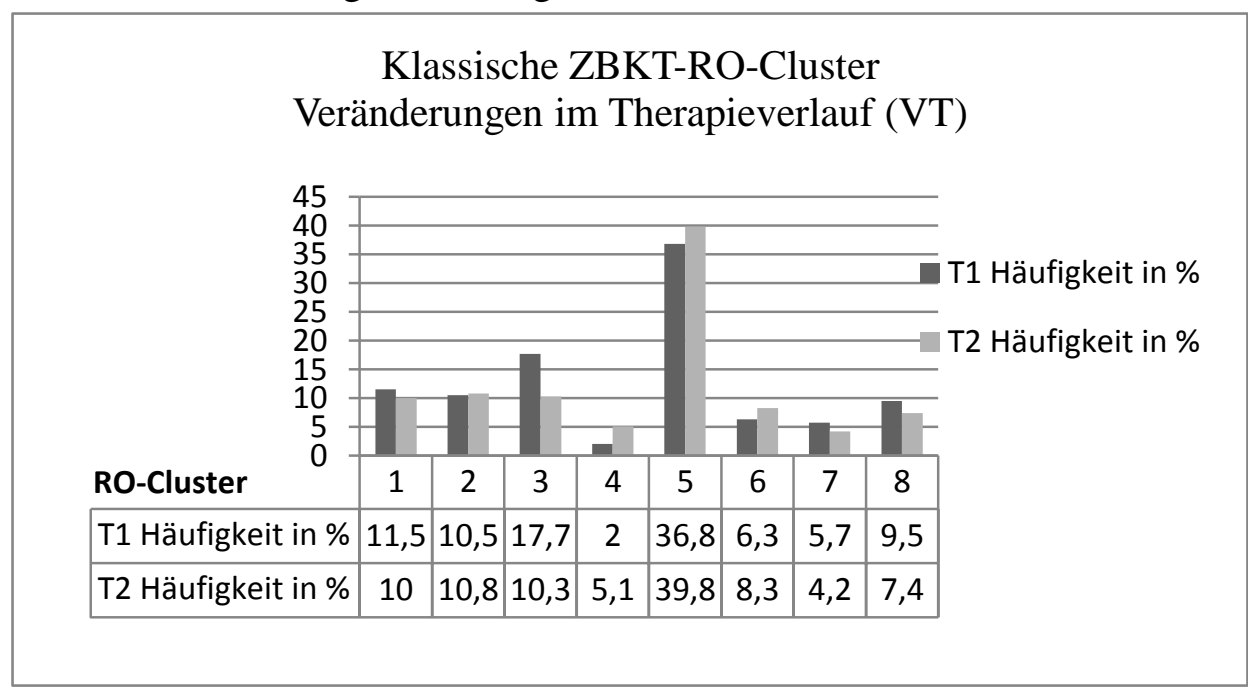

1. Zum Zeitpunkt T1 und T2 ist RO-Cluster 5 am häufigsten ,,andere weisen zurück und sind gegen mich“

2. Stärkste Abnahme der Nennungshäufigkeit während der Therapie: Cluster 3 ,,andere sind bestürzt aufgeregt und ärgerlich“" 
Abb. 47 Veränderung der Häufigkeit klassischer ZBKT-RS-Cluster von VT-Patienten

Klassische ZBKT-RS-Cluster

Veränderungen im Therapieverlauf (VT)

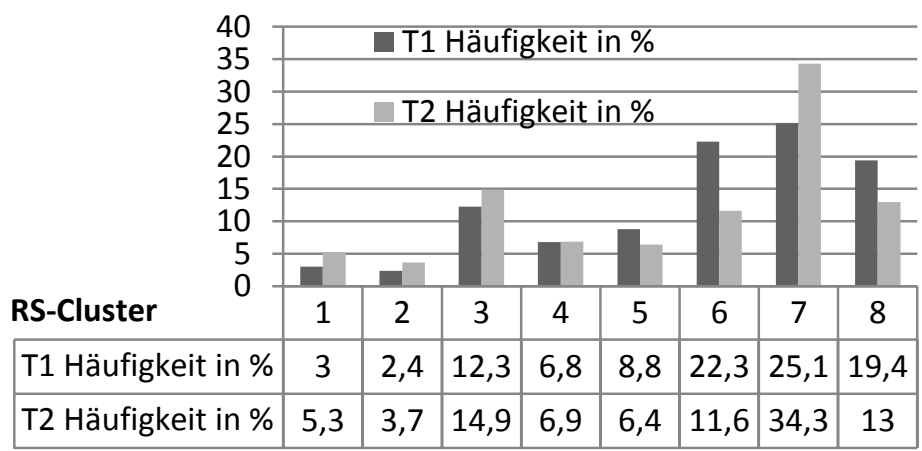

Abb. 48 Visualisierung der Veränderung des RS-Clusters

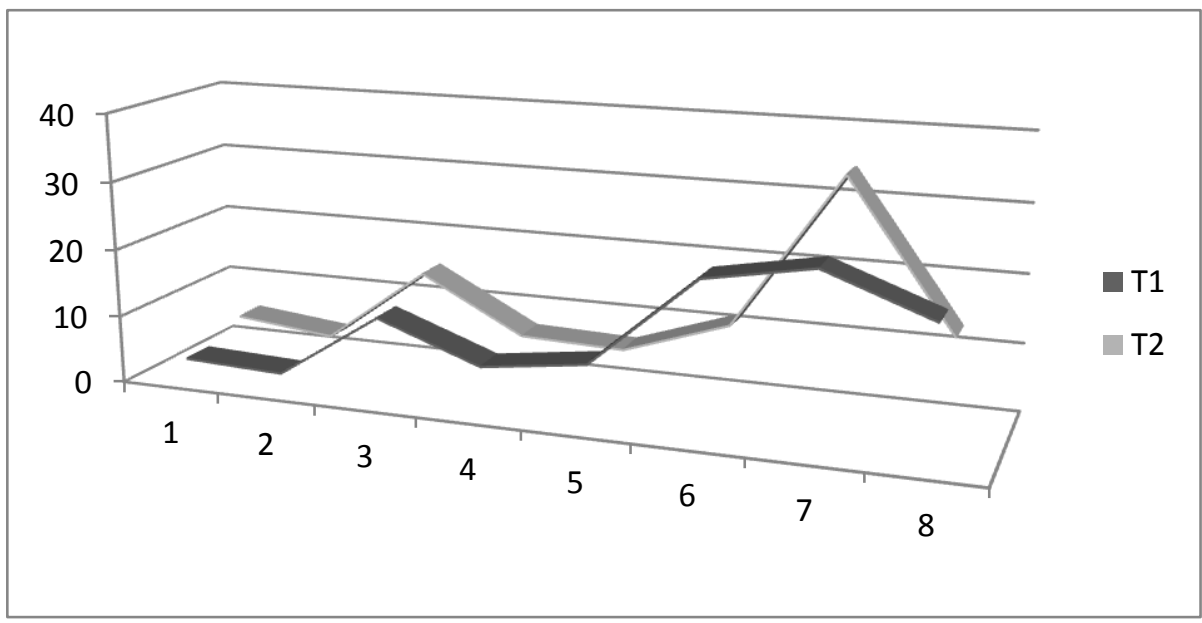

Zum Zeitpunkt T1 sind die RS-Cluster 7, 6 und 8 am häufigsten

1. Cluster 7 ,bin enttäuscht und deprimiert“"

2. Cluster 6 ,ich bin hilflos“

3. Cluster 8 ,,bin ängstlich und beschämt“"

Zum Zeitpunkt T2 sind RS-Cluster 7 und 3 am häufigsten

1. Cluster 7 ,bin enttäuscht und deprimiert“

2. Cluster 3 ,fühle mich respektiert und akzeptiert“

Die stärkste Zunahme der Nennungshäufigkeit während der Therapie

1. Cluster 7 ,bin enttäuscht und deprimiert“

Die stärkste Abnahme der Nennungshäufigkeit während der Therapie

1. Cluster 6 ,fühle mich hilflos“

2. Cluster 8 ,bin ängstlich und beschämt“ 


\subsubsection{Das ZBKT der VT-Patienten zu verschiedenen Zeitpunkten (T1/T2)}

T1:

„Ich möchte mich gut und wohl fühlen, Erfolg und Leistung erreichen und anderen helfen. Andere weisen mich zurück und sind gegen mich. Ich bin enttäuscht und deprimiert, hilflos, ängstlich und beschämt"

\section{T2:}

„Ich möchte Erfolg und Leistung erreichen und anderen helfen. Ich möchte mich gut und wohl fühlen, geliebt und verstanden werden, mich anderen aber auch widersetzen, sie verletzen und kontrollieren. Andere weisen mich zurück und sind gegen mich. Ich bin sehr enttäuscht und deprimiert. Fühle mich aber auch respektiert und akzeptiert" 


\subsection{Die Veränderungen der Häufigkeitsverteilung der LU-Cluster im Therapieverlauf}

\subsubsection{Die Veränderungen bei SET-Patienten}

(Gemessen wurde die prozentuale Häufigkeit eines Clusters zu zwei Therapiezeitpunkten (T1= 3.+5.Therapiestunde, $\mathrm{T} 2=17 .+18$. Therapiestunde))

Abb. 49 Veränderung der Häufigkeit des LU-WO-Clusters von SET-Patienten

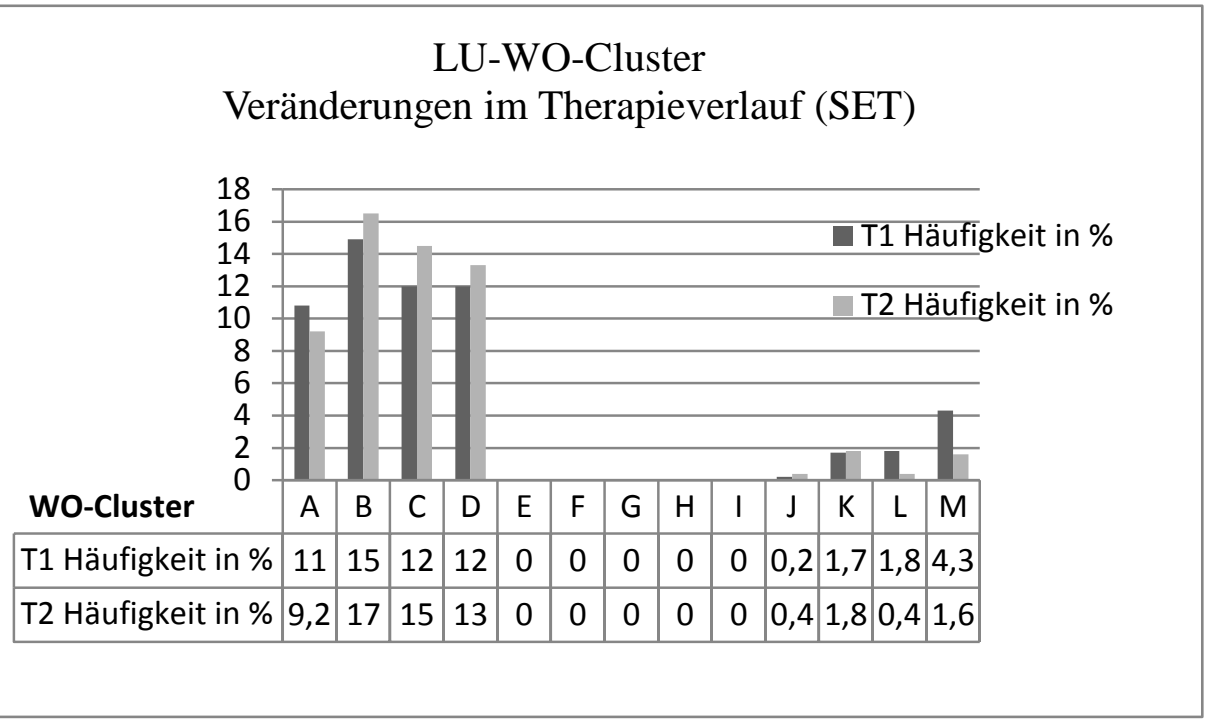

Abb. 50 Visualisierung der Veränderung des WO-Clusters

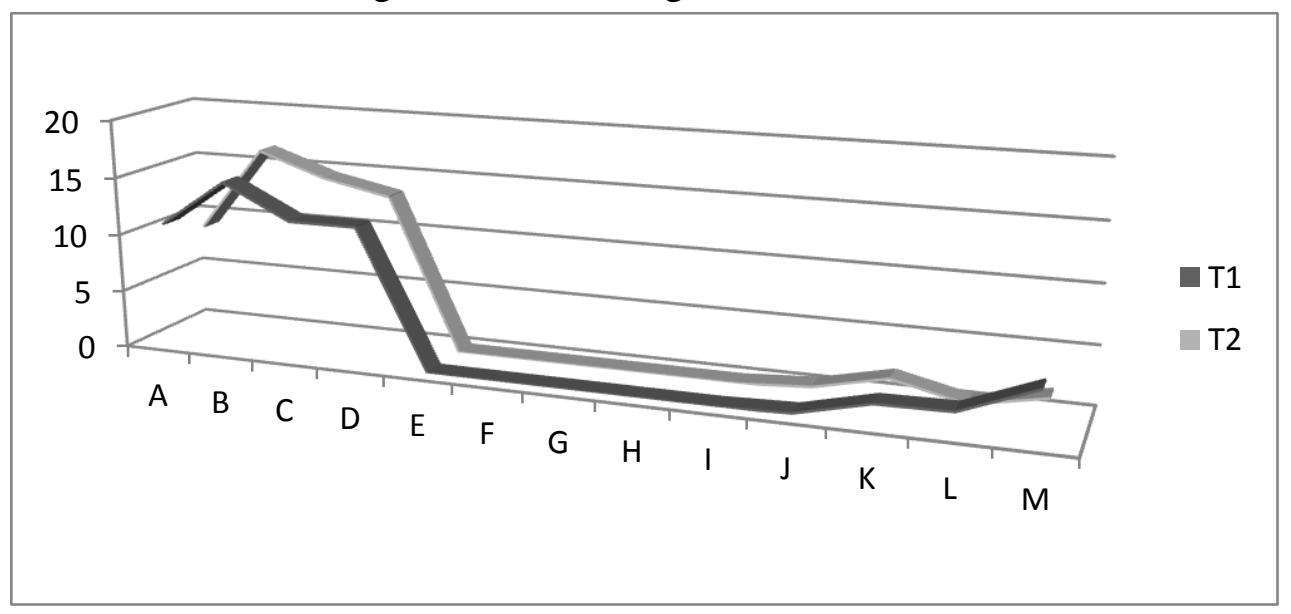

Zum Zeitpunkt T1 und T2 sind die WO-Cluster B, C, D und A am häufigsten

1. B ,unterstützen“

2. C ,lieben, wohl fühlen“

3. D, ,souverän sein“

4. A, ,sich zuwenden“ 
Abb. 51 Veränderung der Häufigkeit des LU-WS-Clusters von SET-Patienten

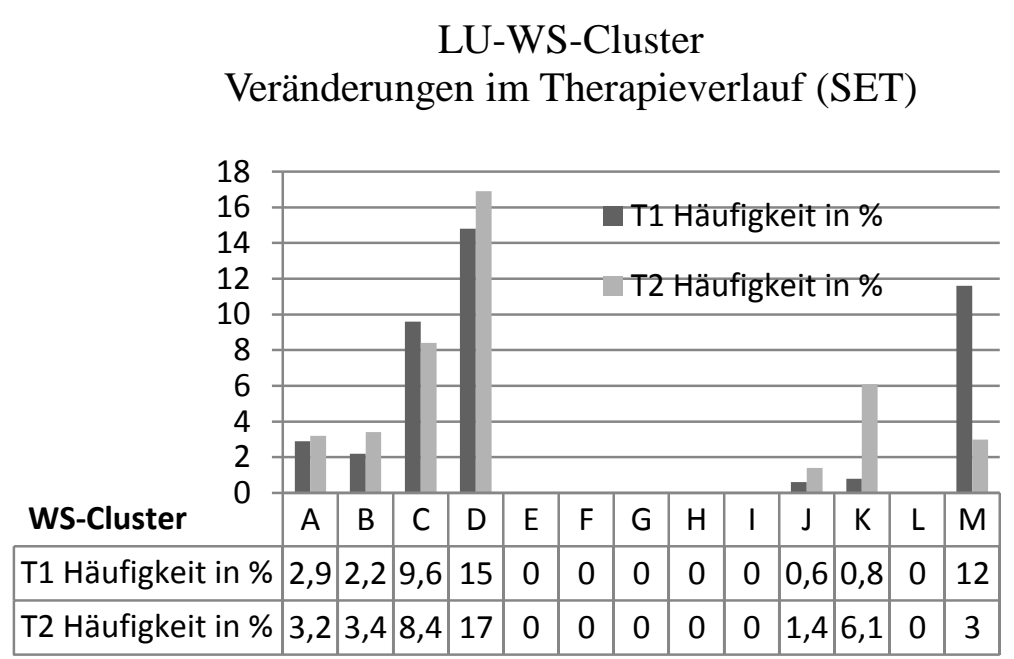

Abb. 52 Visualisierung der Veränderung des WS-Clusters

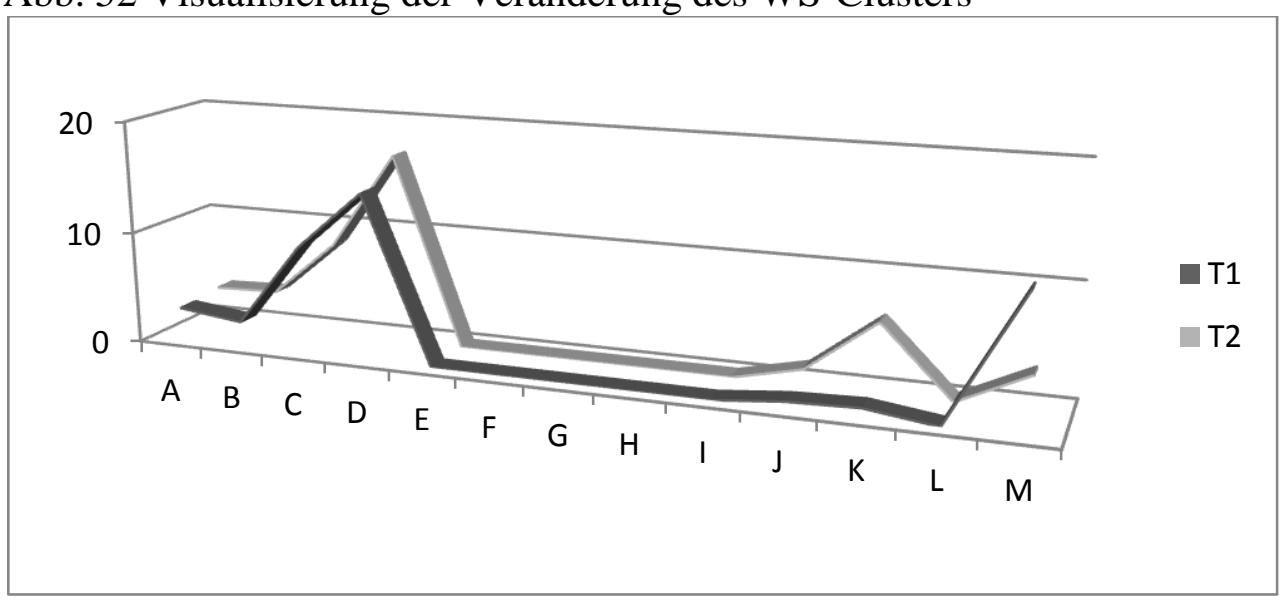

Zum Zeitpunkt T1 sind die WS-Cluster D, M und C am häufigsten

1. D ,souverän sein“

2. $\mathrm{M}$, ,sich zurückziehen“"

3. C ,lieben, wohl fühlen“"

Zum Zeitpunkt T2 sind die WS-Cluster D, C und K am häufigsten

1. D, ,souverän sein“"

2. C ,lieben, wohl fühlen“

3. $\mathrm{K}$,dominieren“"

Stärkste Zunahme der Nennungshäufigkeit während der Therapie

1. K ,dominieren“

Stärkste Abnahme der Nennungshäufigkeit während der Therapie

1. M ,sich zurückziehen“ 
Abb. 53 Veränderung der Häufigkeit des LU-RO-Clusters von SET-Patienten

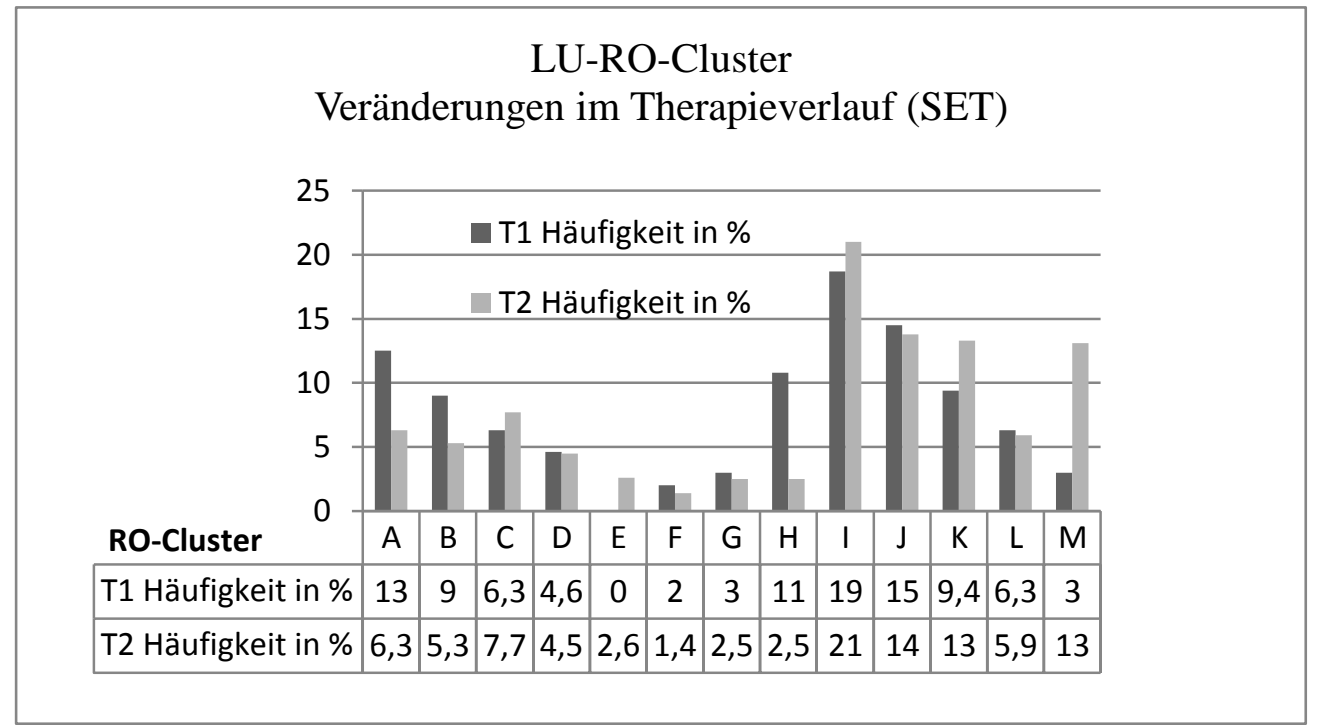

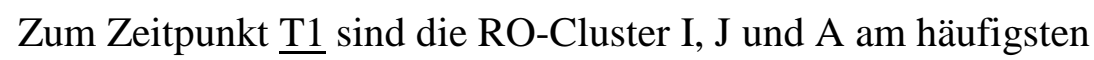

1. I ,unzuverlässig sein“

2. J ,zurückweisen“

3. A, ,sich zuwenden“"

Zum Zeitpunkt $\underline{\mathrm{T} 2}$ sind die RO-Cluster I, J, K und M am häufigsten

1. I ,unzuverlässig sein“"

2. J ,zurückweisen“

3. K, ,dominieren“

4. M ,sich zurückziehen“"

Stärkste Zunahme der Nennungshäufigkeit während der Therapie

1. M ,sich zurückziehen“"

2. K ,dominieren“

Stärkste Abnahme der Nennungshäufigkeit während der Therapie

1. H ,verärgert, unsympathisch sein“"

2. A, ,sich zuwenden“ 
Abb. 54 Veränderung der Häufigkeit des LU-RS-Clusters von SET-Patienten

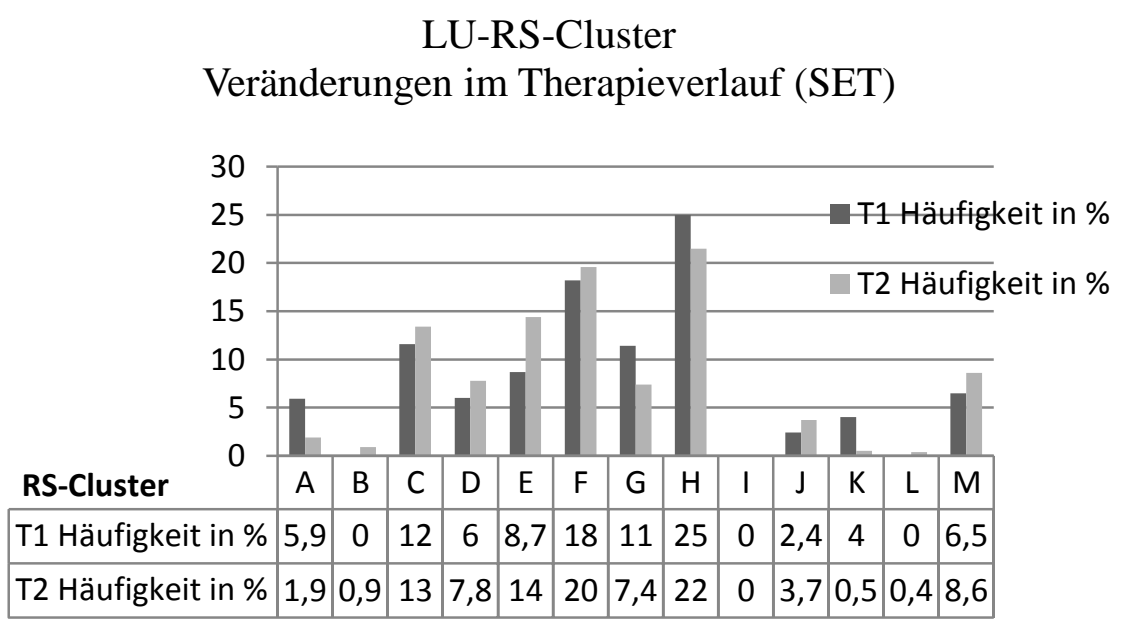

Abb. 55 Visualisierung der Veränderung des RS-Clusters

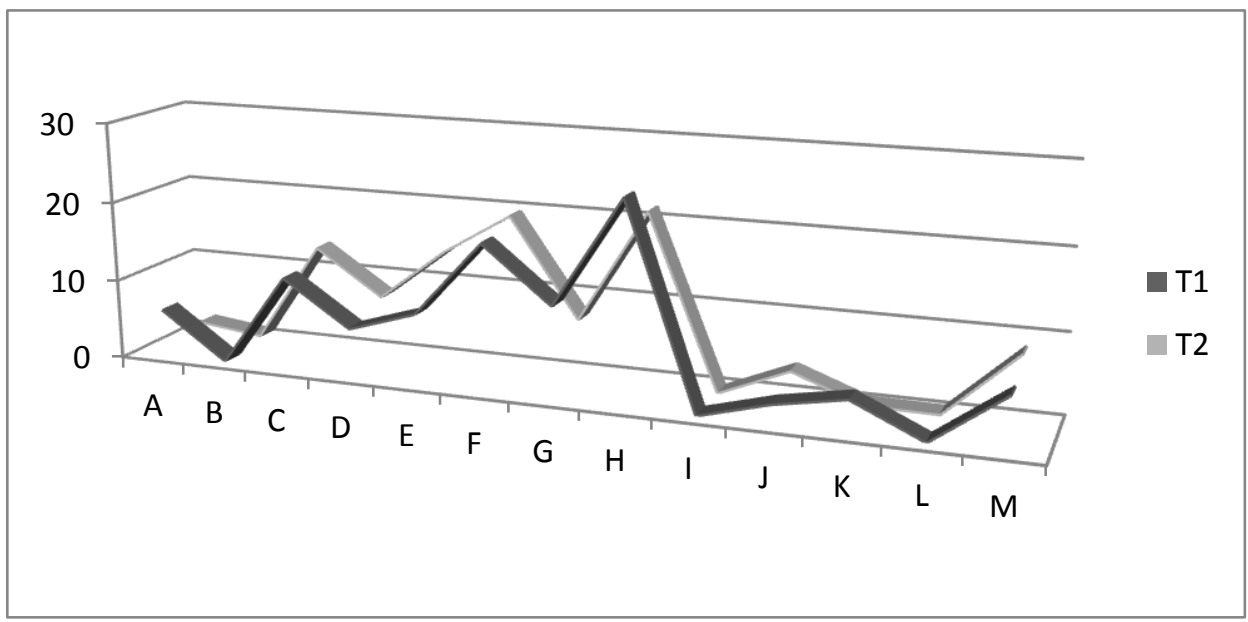

Zum Zeitpunkt T1 sind die RS-Cluster H, F, C und G am häufigsten

1. $\mathrm{H}$,verärgert, unsympathisch sein“"

2. F ,unzufrieden sein, Angst haben“

3. C ,lieben, wohl fühlen“

4. G, ,fremdbestimmt sein“

Zum Zeitpunkt T2 sind die RS-Cluster H, F, E und C am häufigsten

1. $\mathrm{H}$, verärgert, unsympathisch sein“"

2. F ,unzufrieden sein“"

3. E, ,depressiv sein“

4. C ,lieben, wohl fühlen“"

Stärkste Zunahme der Nennungshäufigkeit während der Therapie

1. E ,depressiv sein“

Stärkste Abnahme der Nennungshäufigkeit während der Therapie

1. A ,sich zuwenden“"

2. $\mathrm{K}$, dominieren“

3. $\mathrm{G}$,fremdbestimmt sein“ 


\subsubsection{Das LU-ZBKT der SET-Patienten zu verschiedenen Zeitpunkten (T1/T2)}

\section{T1:}

„Andere sollen mich unterstützen, souverän sein, mich lieben und sich mir zuwenden. Ich möchte souverän sein, mich zurückziehen, lieben und mich wohl fühlen. Andere sind unzuverlässig". Ich bin verärgert, unzufrieden und habe Angst"

T2:

„Andere sollen mich unterstützen, mich lieben, souverän sein und sich mir zuwenden. Ich möchte souverän sein, mich wohl fühlen und dominieren. Andere sind unzuverlässig. Ich bin verärgert und unzufrieden, habe Angst“ 


\subsubsection{Die Veränderungen bei VT-Patienten}

Gemessen wurde die prozentuale Häufigkeit eines Clusters zu zwei Therapiezeitpunkten (T1= 3.+5.Therapiestunde, $\mathrm{T} 2=17 .+18$. Therapiestunde)

Abb. 56 Veränderung der Häufigkeit des LU-WO-Clusters von VT-Patienten

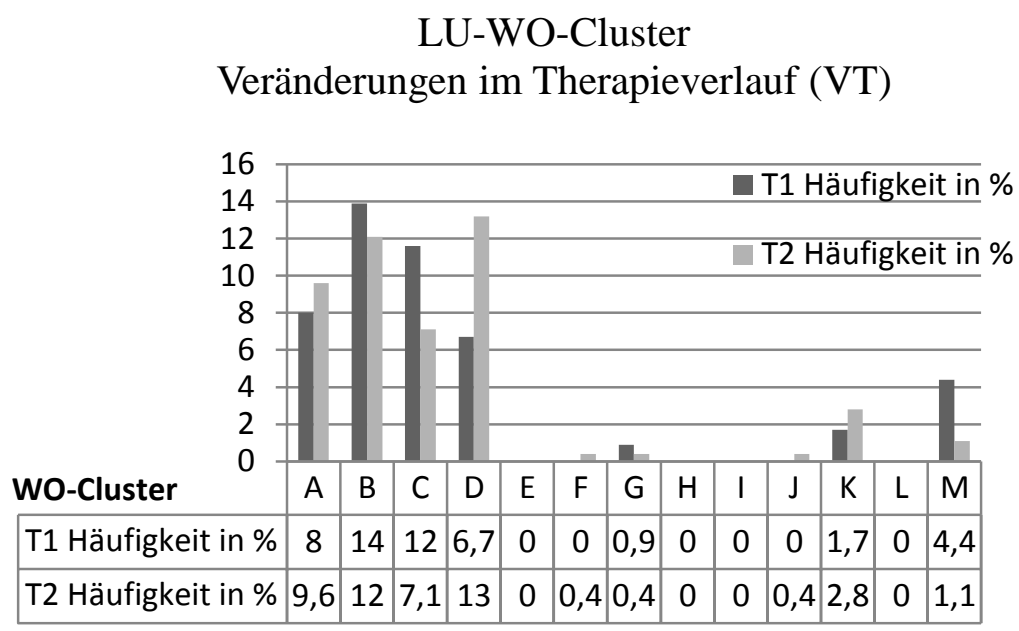

Abb. 57 Visualisierung der Veränderung des WO-Clusters

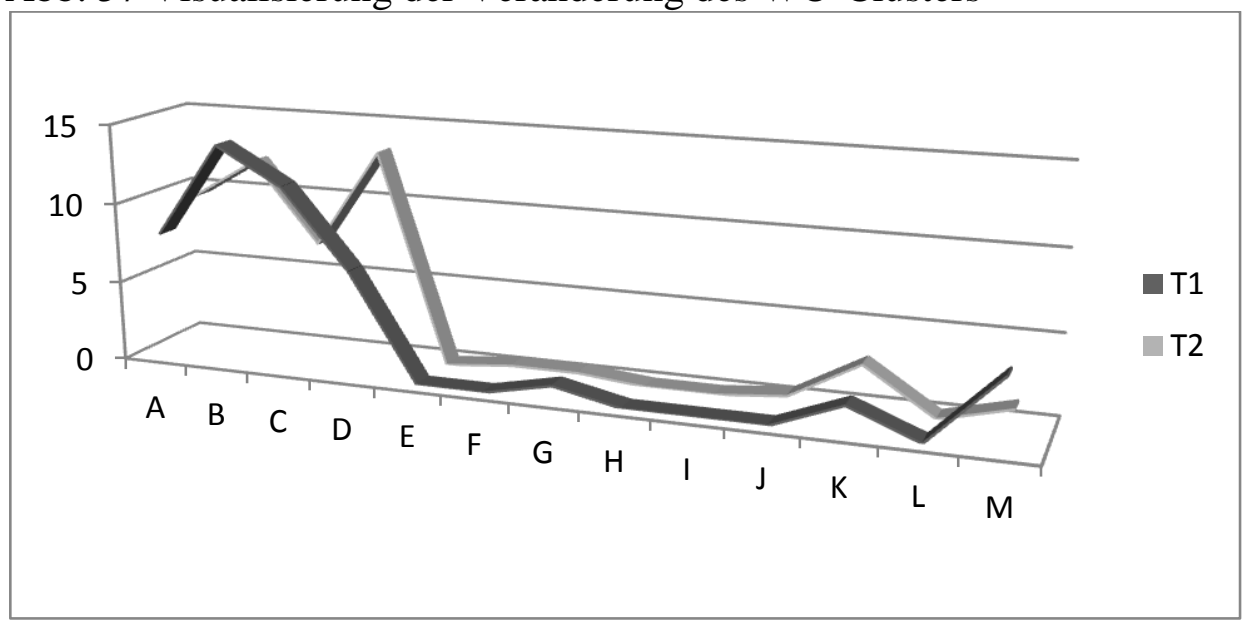

Zum Zeitpunkt T1 und T2 sind die WO-Cluster_B, C, A und D am häufigsten

1. B ,unterstützen“"

2. C ,lieben, wohl fühlen“

3. A, ,sich zuwenden“"

4. D ,souverän sein“

Stärkste Zunahme der Nennungshäufigkeit während der Therapie

1. D ,souverän sein“"

Stärkste Abnahme der Nennungshäufigkeit während der Therapie

1. C ,lieben, wohl fühlen“

2. M, ,zurückziehen“ 
Abb. 58 Veränderung der Häufigkeit des LU-WS-Clusters von VT-Patienten

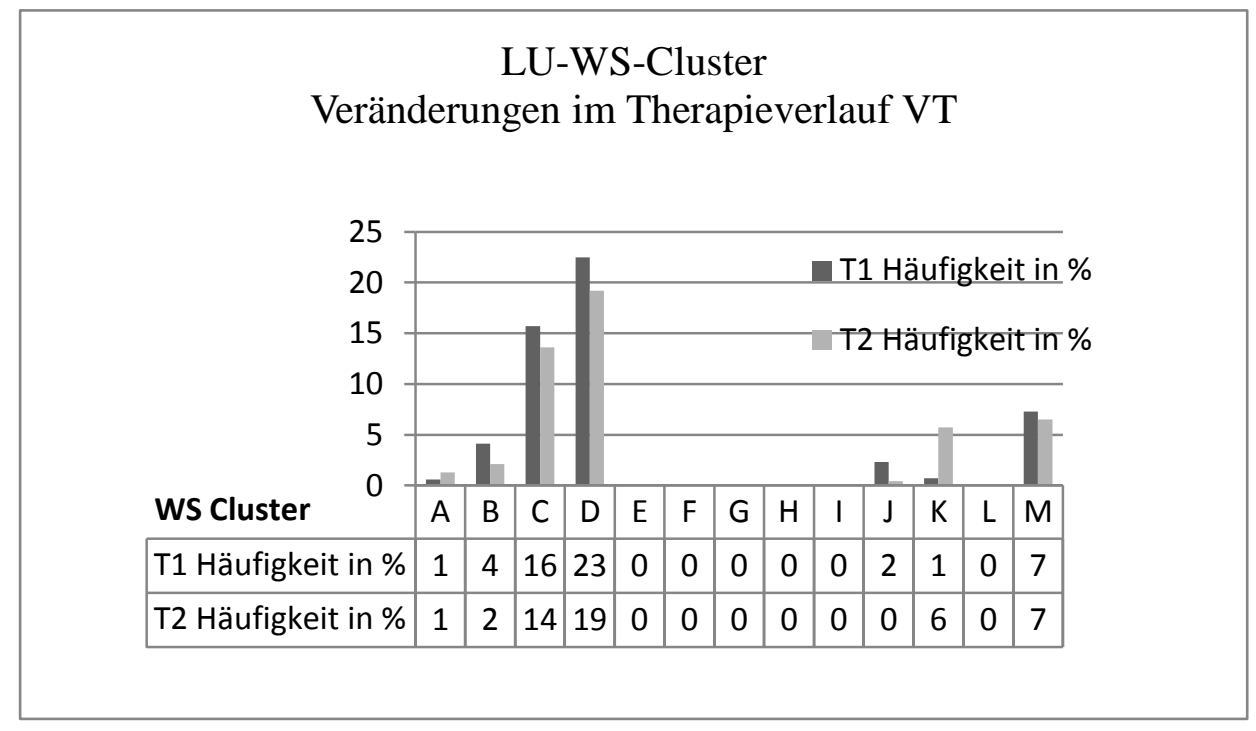

Abb. 59 Visualisierung der Veränderung des WS-Clusters

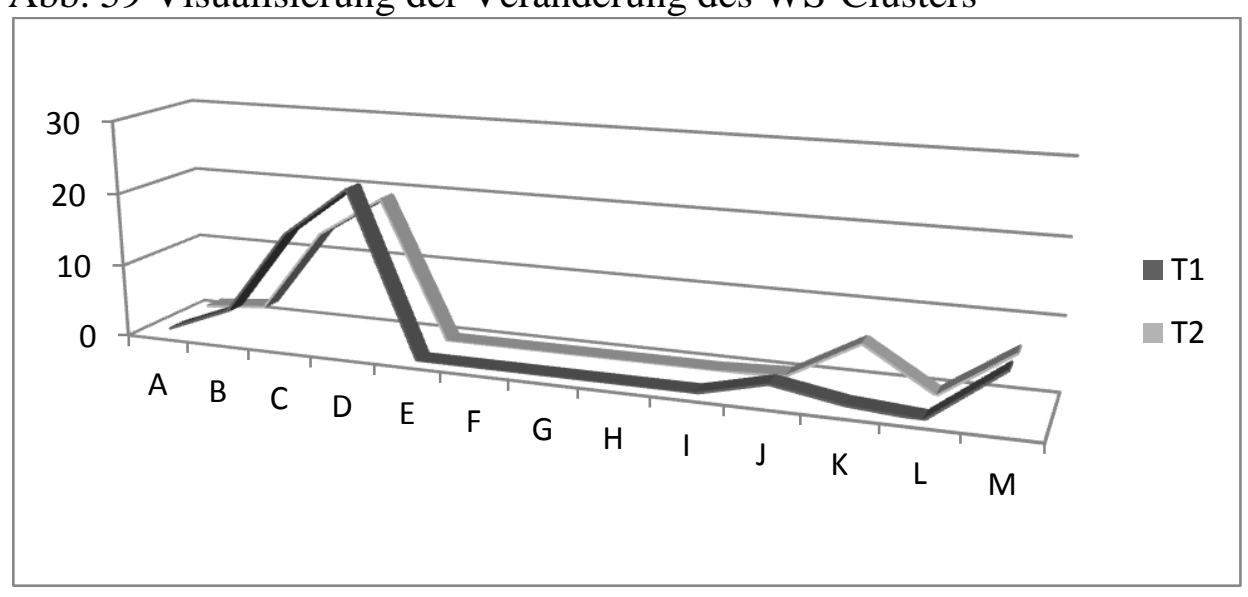

Zum Zeitpunkt T1 sind die WS-Cluster D, C und M am häufigsten

1. D ,souverän sein“

2. C ,lieben, sich wohl fühlen“

3. M, ,sich zurückziehen“

Zum Zeitpunkt T2 sind die WS-Cluster D, C, K und M am häufigsten

1. D ,souverän sein“"

2. C ,lieben, sich wohl fühlen“"

3. K ,dominieren“

4. $M$,sich zurückziehen“"

Stärkste Zunahme der Nennungshäufigkeit während der Therapie

1. K ,dominieren“

Stärkste Abnahme der Nennungshäufigkeit während der Therapie

1. D ,souverän sein“ 
Abb. 60 Veränderung der Häufigkeit des LU-RO-Clusters von VT-Patienten

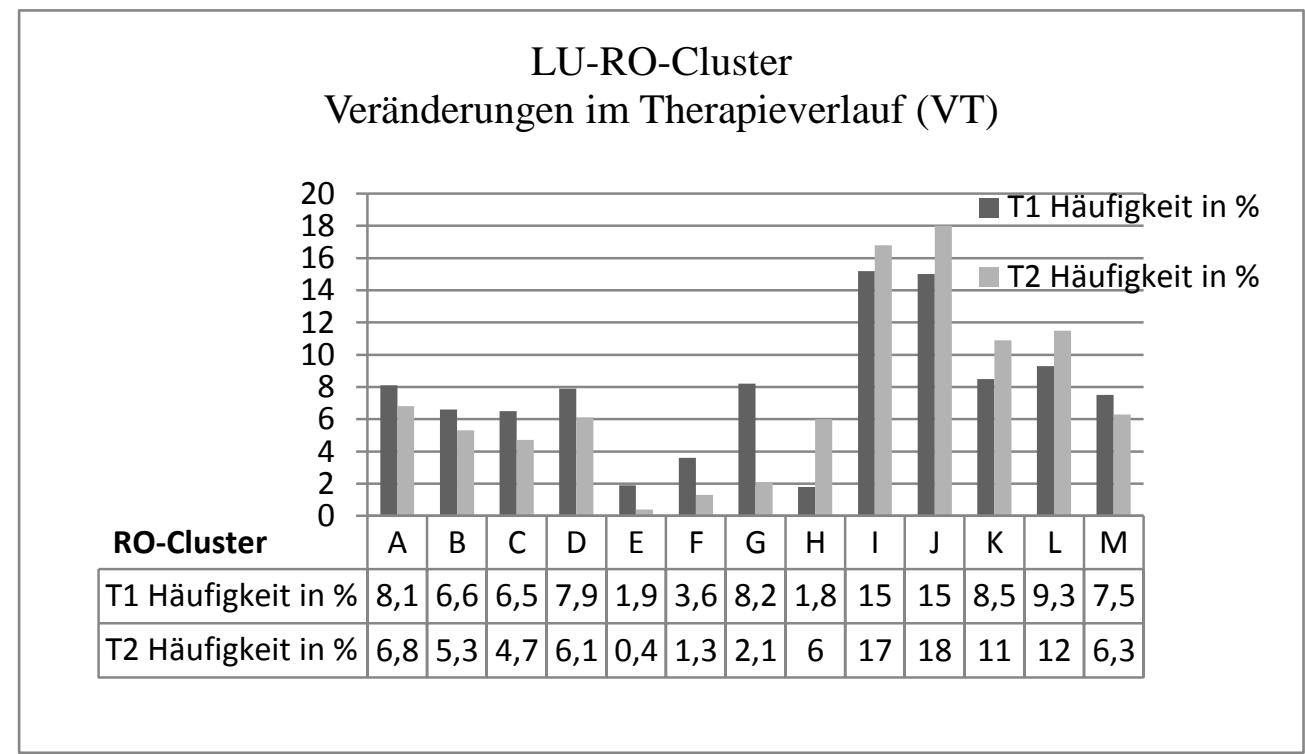

Zum Zeitpunkt $\underline{\mathrm{T} 1 \mathrm{und} \mathrm{T} 2}$ sind die $\underline{\mathrm{RO}-\mathrm{Cluster}} \mathrm{I}$ und $\mathrm{J}$ am häufigsten

1. I ,unzuverlässig sein“

2. J ,zurückweisen“

Stärkste Zunahme der Nennungshäufigkeit während der Therapie

1. $\mathrm{H}$, verärgert, unsympathisch sein“"

Stärkste Abnahme der Nennungshäufigkeit während der Therapie

1. G ,fremdbestimmt sein“ 
Abb. 61 Veränderung der Häufigkeit des LU-RS-Clusters von VT-Patienten

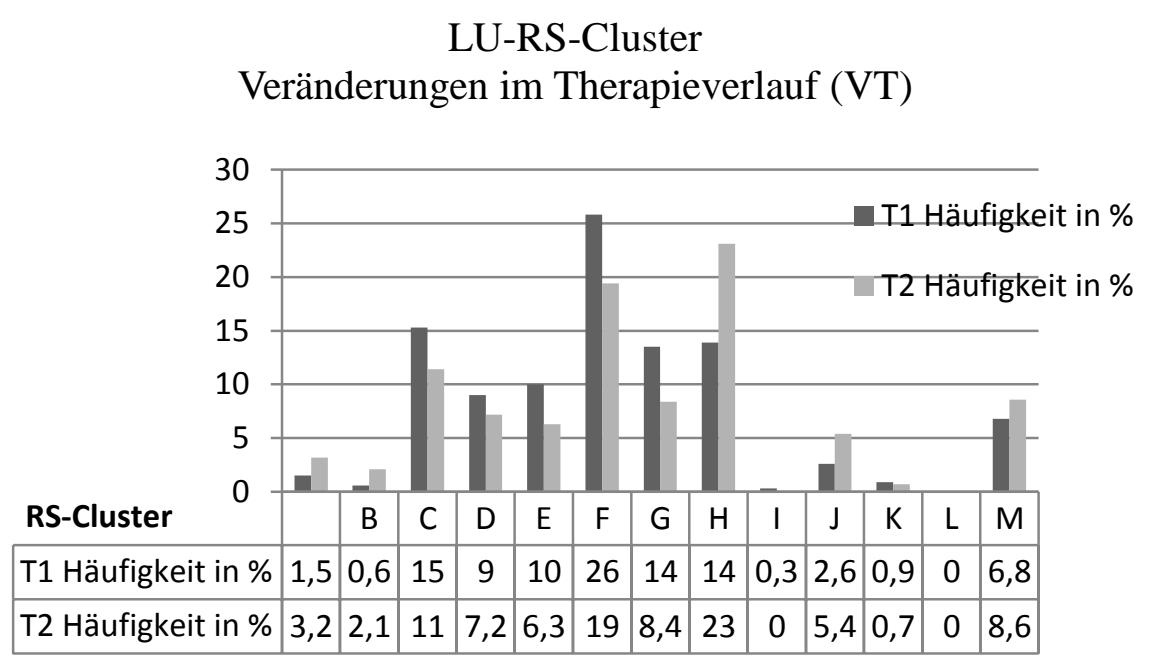

Abb. 62 Visualisierung der Veränderung des RS-Clusters bei VT-Patienten

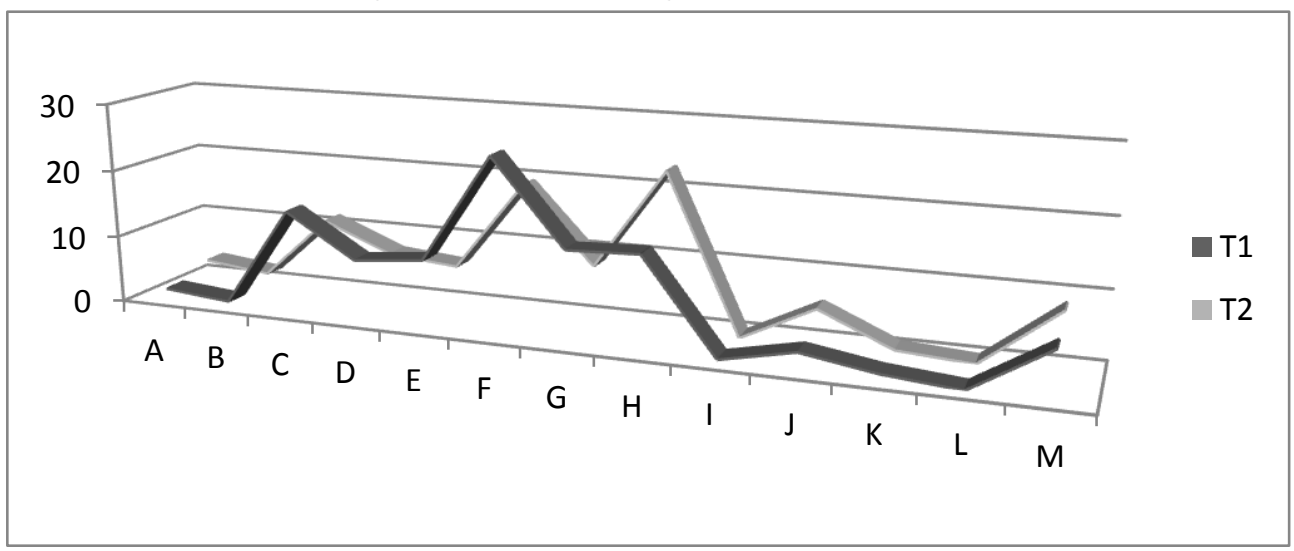

Zum Zeitpunkt T1 sind die RS-Cluster F, C, G und $\mathrm{H}$ am häufigsten

1. F ,unzufrieden sein, Angst haben“"

2. C ,lieben, sich wohl fühlen“"

3. G ,fremdbestimmt sein“

4. H ,verärgert, unsympathisch sein“"

Zum Zeitpunkt T2 sind die RS-Cluster H, F, C und M am häufigsten

1. $\mathrm{H}$,,verärgert, unsympathisch sein“"

2. F ,unzufrieden sein, Angst haben“"

3. C ,lieben, sich wohl fühlen“"

4. M, ,sich zurückziehen“"

Stärkste Zunahme der Nennungshäufigkeit während der Therapie

1. $\mathrm{H}$,,verärgert, unsympathisch sein“"

Stärkste Abnahme der Nennungshäufigkeit während der Therapie

1. F ,unzufrieden sein, Angst haben“"

2. $\mathrm{G}$, ,fremdbestimmt sein“

3. E ,depressiv sein“

4. C ,lieben, sich wohl fühlen“ 


\subsubsection{Das LU-ZBKT der VT-Patienten zu verschiedenen Zeitpunkten (T1/T2)}

$\mathrm{T} 1$ :

WO-AV: „Andere sollen mich unterstützen und lieben “

$W S-A V:$,Ich möchte souverän sein, lieben und mich wohlfühlen “

$R O-A V$ : „Andere sind unzuverlässig und weisen zurück"

RS-AV: „Ich bin unzufrieden und habe Angst“

T2:

WO-AV: „Andere sollen souverän sein, mich unterstützen, sich mir zuwenden und mich lieben"

WS-AV: „Ich möchte souverän sein, lieben und mich wohl fühlen, dominieren und mich zurückziehen können "

$R O-A V$ : „Andere sind unzuverlässig und weisen mich noch stärker zurück“

$R S-A V$ : ,Ich bin verärgert und unzufrieden “ 


\subsection{Das maßgeschneiderte ZBKT}

Der letzte Ergebnisteil befasst sich mit der fünften allgemeinen Fragestellung der Dissertation. Lassen sich zentrale Themen und krankheitstypische Muster mit ausgezählten oder maßgeschneiderten ZBKT`s besser erkennen? Unterscheiden sich maßgeschneiderte ZBKT`s von VT- und SET-Patienten?

Während bei den ausgezählten ZBKT`s der Aspekt der Häufigkeit, also gewissermaßen der Quantität eines Clusters eine besondere Rolle spielt, kommt es beim Erstellen der maßgeschneiderten ZBKT`s in besonderem Maße darauf an, einen roten Faden aufzuspüren, welcher sich durch viele Wünsche und Reaktionen eines Patienten oder auch einer ganzen Gruppe von Menschen zieht. Es wird also eine übergeordnete Formulierung gesucht, welche für möglichst viele Wünsche und Reaktionen zutreffen soll. Hierzu wurde entgegengesetzt zum Vorgehen beim AV nicht einfach quantitativ erfasst, sondern qualitativ aufgespürt. Dieser Schritt erfolgt direkt am Narrativ und spart hierdurch die Übersetzung in SK und CL vollständig aus. Klinisch ist das TM-ZBKT der Patientenaussage somit wesentlich näher.

Das TM-ZBKT wurde exemplarisch für jeweils 10 SET- und VT-Patienten erstellt. Erneut stellten wir uns die Frage, ob sich Unterschiede zwischen VT- und SET-Patienten aufzeigen lassen. Im Folgenden findet sich die maßgeschneiderte Formulierung für jede ZBKTKomponente im prä/post-Vergleich als Etappen-ZBKT des Therapieanfanges $(3 .+5$. Therapiestunde $=\mathrm{T} 1)$ und Therapieendes $(17 .+18$. Therapiestunde $=\mathrm{T} 2)$.

\subsubsection{TM-ZBKT der SET-Patienten (T1/T2)}

*erstellt aus Daten von 10 SET-Patienten

\section{$\underline{\text { Patient 1: }}$}

W: (T1) will unabhängig und selbständig sein und doch für die anderen da sein / (T2) aufraffen, nicht egoistisch sein, Kontakt knüpfen

RO: (T1) bewegt nicht eine Stecknadel, andere beanspruchen mich sehr / (T2) wollten uns nicht dabei haben

RS: (T1) bin deprimiert und enttäuscht / (T2) habe Angst, komme nicht zurecht, habe durchgehangen

\section{$\underline{\text { Patient 2: }}$}

W: (T1) will bürgerliches Leben, alles richtig machen, aber keine Langeweile / (T2) ich will klare Positionen und meine Ruhe haben, andere sollen tun was ich sage

RO: (T1) haben Freiheitsdrang, sind unzuverlässig, gleichgültig/ (T2) andere ignorieren meine Wünsche, ziehen sich zurück, bedrohen mich

RS: (T1) kann es nicht mehr ertragen, bin verzweifelt und will es nicht wahr haben / (T2) fühle mich ausgeliefert, hilflos, ist nicht das was ich will 


\section{Patient 3:}

W: (T1) alles retten, was vom Aussterben bedroht ist, alles akkurat und souverän erledigen / (T2) andere nicht hängen lassen, helfen, dabei aber selbst nicht im Vordergrund stehen

RO: (T1) andere lassen mich im Stich und verstehen mich nicht / (T2) fühlen sich vor den Kopf gestoßen

RS: (T1) bin deprimiert und ängstlich, aber so kann es nicht bleiben / (T2) steigere mich rein, mische mich ein, belastet mich

\section{Patient 4:}

W: (T1) will meinen Mann stehen und das tun, wozu ich Lust habe und dabei anderen gefallen / (T2) andere sollen mich respektvoll behandeln und tun was ich sage

RO: (T1) sind unsensibel und interessiert die gar nicht / (T2) haben mich im Stich gelassen, gehen nicht auf mich ein

RS: (T1) ging mir richtig schlecht, war mir zu viel / (T2) traurig, wütend unzufrieden

\section{$\underline{\text { Patient 5: }}$}

W: (T1) wünsche mir Nähe, Respekt und Anerkennung / (T2) will attraktiv sein, Anerkennung, sexuelle Kontakte

RO: (T1) setzen mich unter Druck, respektieren mich nicht / (T2) fordern, setzen mich unter Druck, weisen zurück

RS: (T1) bekomme Angst, bin verunsichert / (T2) bin überfordert, ängstlich

\section{$\underline{\text { Patient 6: }}$}

W: (T1) jemand der mich versteht, geborgen sein, nicht auffallen wollen / (T2) ich möchte eine aufregende Beziehung führen und ungebunden, frei sein

RO: (T1) sind unberechenbar, ekeln sich oder loben mich / (T2) sind lieb und nett aber langweilig

RS: (T1) schäme mich oder bin erleichtert, bin abhängig von der Reaktion anderer / (T2) bin dankbar, aber sehe keinen Sinn, bin unzufrieden

\section{$\underline{\text { Patient } 7:}$}

W: (T1) möchte Ausmaß der emotionalen Nähe zu anderen selbst bestimmen / (T2) möchte nicht alleine sein, beschützt werden, meine Bedürfnisse durchsetzen

RO: (T1) machen es gern dramatisch, sind vorwurfsvoll / (T2) sind rücksichtslos, nicht an meinen Wünschen interessiert

RS: (T1) reingesteigert und dann über mich selbst geärgert / (T2) bin ängstlich, verärgert und wütend

\section{$\underline{\text { Patient 8: }}$}

W: (T1) für mich einsetzen, Nähe zu anderen finden / (T2) souverän sein, stark sein, durchsetzen

RO: (T1) spenden Nähe, setzen sich mit mir auseinander / (T2) sind distanziert

RS: (T1) bin zufrieden / (T2) trotz einiger Erfolge bin ich unzufrieden 


\section{$\underline{\text { Patient 9: }}$}

W: (T1) andere sollen mich respektieren und richtig behandeln / (T2) möchte tun, wozu ich Lust habe und unterstützt werden

RO: (T1) sind nicht verfügbar, verstehen nicht / (T2) nehmen nicht immer Rücksicht

RS: (T1) ärgert mich / (T2) bin verärgert, ziehe mich zurück

\section{$\underline{\text { Patient 10: }}$}

W: (T1) tun was ich für richtig halte, durchsetzen / (T2) mich auf andere verlassen können

RO: (T1) nehmen meine Wünsche nicht wahr / (T2) entziehen sich ihrer Verantwortung

RS: (T1) unsicher unzufrieden / (T2) lasse andere nicht hängen (ein lachendes ein weinendes Auge)

\section{TM-ZBKT:}

T1: „Ich will es richtig machen, sodass andere mich respektieren. Andere sind unzuverlässig und gleichgültig, tun nicht so, wie ich will. Ich bin enttäuscht, verunsichert, ärgerlich und bekomme Angst"

T2: „Ich möchte stark sein, andere sollen mir nahe sein und tun, was ich sage. Andere sind distanziert und machen nicht mit. Ich bin ärgerlich, enttäuscht und bekomme Angst “ 


\subsubsection{TM-ZBKT der VT-Patienten (T1/T2)}

* erstellt aus Daten von 10 VT-Patienten

Patient 1:

W: (T1) meine Ruhe haben / (T2) von anderen unabhängig sein, selbstsicherer und selbstbewusster werden

RO: (T1) den anderen stößt etwas zu / (T2) andere respektieren mich nicht, sind mir überlegen

RS: (T1) Angst / (T2) stark im Dunkeln gefühlt, hat mir weh getan, bin gekränkt

\section{Patient 2:}

W: (T1) möchte selbstbewusst verteidigen / (T2) ausserhalb der Situation bleiben, Auseinandersetzung vermeiden

RO: (T1) andere verstehen mich nicht, agieren gegen mich / (T2) sind rechthaberisch, brechen Beziehung ab

RS: (T1) ärgere mich furchtbar / (T2) bin ängstlich, unzufrieden

\section{$\underline{\text { Patient 3: }}$}

W: (T1) will gut sein und das Richtige tun / (T2) ich möchte mit jedem gut auskommen, respektiert werden

RO: (T1) sind mir wohlgesonnen, an mir interessiert / (T2) möchten mich loswerden

RS: (T1) bin nervös und verunsichert / (T2) fühle mich unwohl, bin unsicher

\section{Patient 4:}

W: (T1) ich möchte andere beschützen, für sie Konflikte lösen / (T2) friedliche Kommunikation mit anderen, liebevoll sein

RO: (T1) andere weisen mich zurück, sind nicht verfügbar / (T2) sind ärgerlich unberechenbar

RS: (T1) bin ängstlich und verunsichert / (T2) bin hilflos, ziehe mich zurück

\section{$\underline{\text { Patient 5: }}$}

W: (T1) Idealismus, klare Linie / (T2) Nicht auf Diskussionen einlassen

RO: (T1) andere kritisieren mich, brauchen mich nicht / (T2) sind beherrschend

RS: (T1) ich bin wütend und zweifle / (T2) setze mich durch, bin zufrieden

\section{$\underline{\text { Patient 6: }}$}

W: (T1) mich gut fühlen und entspannen / (T2) mich entspannen, Dinge gut machen

RO: (T1) andere sind distanziert / (T2) andere belasten und belästigen mich

RS: (T1) Angst Anspannung / (T2) fühle mich unwohl, deprimiert 


\section{Patient 7:}

W: (T1) will stark sein und von anderen beschützt werden / (T2) erfolgreich und glücklich sein

RO: (T1) andere sind abwesend, respektieren mich nicht / (T2) setzen mich unter Druck

RS: (T1) ärgere mich, tut mir nicht gut / (T2) bin deprimiert und wütend

\section{Patient 8:}

W: (T1) anderen wichtig sein / (T2) tapfer sein und beschützt werden

RO: (T1) interessieren sich für mich / (T2) sind rücksichtlos, verletzten mich, manche nehmen mich an

RS: (T1) total fidel / (T2) bin überwiegend hilflos und ängstlich, über Zuwendung freue ich mich

\section{$\underline{\text { Patient 9: }}$}

W: (T1) mich wehren, nach meinen Vorstellungen leben / (T2) mich zurücknehmen, nett sein RO: (T1) sind gegen mich / (T2) sind unzuverlässig

RS: (T1) bin verärgert / (T2) bin wütend und traurig

\section{Patient 10:}

W: (T1) nicht das Leben leben, das die Eltern vorschlagen / (T2) ihm in den Arsch treten RO: (T1) nicht an mir interessiert / (T2) sitzt am längeren Hebel

RS: (T1) bin enttäuscht und verzweifelt / (T2) muss mich nach ihm richten

\section{$\underline{T M-Z B K T:}$}

T1: „Ich will gut sein, damit andere mich respektieren und beschützen. Andere sind unzuverlässig, uninteressiert und gegen mich. Ich bin ärgerlich, enttäuscht, unsicher und bekomme Angst“

T2: „Ich will es gut machen und stark sein, dass andere mich mögen. Andere sind unzuverlässig und handeln gegen mich, ich in deprimiert und wütend" 


\section{Diskussion}

\subsection{Patientengruppe, Material und Therapieverfahren}

Die Stichprobe von 45 Patienten zeigt eine gute innere Variabilität, welche sich unter anderem auf eine recht breite Streuung des Alters der Patienten (18-65 Jahre, durchschnittlich 43,46 Jahre), unterschiedliche Ausbildungsstände und berufliche Qualifikationen stützt. 80 \% der Patienten sind Frauen, was durchaus der Realität der Geschlechterverteilung in der psychotherapeutischen Praxis entspricht. Hinsichtlich der krankhaften, dem Grundleiden zugrunde liegenden Symptome handelt es sich um eine homogene Patientengruppe. Zur Beantwortung unserer Fragen ist die untersuchte Stichprobe daher gut geeignet.

Die vorliegende Dissertation konzentriert sich auf die Auswertung von Psychotherapiestunden von insgesamt 45 Patienten mit der Hauptdiagnose GAS. Die Diagnostik erfolgte mit Hilfe strukturierter, klinischer Interviews (SKID I, II) (Wittchen et al. 1997) nach DSM IV. Die Interviews wurden von trainierten und erfahrenen Interviewern vorgenommen, die Diagnosen in Übereinstimmung von mindestens zwei der Interviewer getroffen. Das auszuwertende Material umfasste 171 Therapiesitzungen mit insgesamt 802 Narrativen. Die Narrative der dritten und fünften, sowie der siebzehnten und achtzehnten Therapiestunde wurden zusammengefasst, um Etappen-ZBKT`s mit einer ausreichend hohen Anzahl an BE`s erstellen zu können.

Zusammenfassend handelt es sich um eine ausreichend große, diagnostisch homogene und sorgfältig diagnostizierte Patientengruppe.

20 der 45 Patienten wurden psychodynamisch behandelt. Basierend auf dem supportiv expressiven Therapieansatz (SET) nach einem Manual Luborskys (1999) erhielten diese Patienten eine psychoanalytisch orientierte Fokaltherapie für GAS-Patienten (Leichsenring et al. 2005). Die übrigen 25 Patienten erhielten eine VT. Beide Therapieverfahren waren als ambulante Kurzzeittherapie konzipiert.

Dem Ergebnisteil ist zu entnehmen, wie viele Therapiesitzungen insgesamt ausgewertet wurden und wie viele BE`s darin enthalten waren. Sowohl in VT-, als auch in SET-Sitzungen konnten genügend BE`s zur Bestimmung des ZBKT`s gefunden werden. Das durchschnittliche ZBKT der VT basiert auf 14,5 BE`s, das der SET auf 21,95 BE`s. Die verschieden hohe Anzahl liegt im Aufbau des jeweiligen Therapieverfahrens begründet. Während in SET-Sitzungen der Beziehungsaspekt und damit einhergehend Erzählungen über Beziehungen mit und zu anderen, beziehungsweise mit und zu sich selbst deutlicher zu Tage treten, richtet die VT das Augenmerk auf akute Problembewältigung, z.B. in Form von Entspannungsübungen. VT-Sitzungen sind daher automatisch ärmer an BE`s. 


\subsection{Die ZBKT-Methode}

Die von Lester Luborsky etablierte Methode zur Bestimmung des ZBKT`s (1977, 1998a) ist ein inzwischen vielfach erprobtes Instrument zur Messung von Übertragung in Beziehungen. Albani et al. (2008, S. 127) gehen davon aus, dass mit der ZBKT-Methode strukturelle Aspekte des klinischen Übertragungskonzeptes in Form internalisierter Beziehungsmuster erfasst werden können. Dabei sind allerdings nur manifeste Themen greifbar, während Unbewusstes nicht erfasst werden kann.

Die ZBKT-Methode wird heute zur Behandlung einer breitgefächerten Patientengruppe eingesetzt. Ein großer Vorteil der Methode besteht darin, dass Patientenaussagen nahezu ungefiltert in die Auswertung einfließen und somit der individuellen Aussage eines jeden Patienten Rechnung getragen wird (Albani et al. 2008). Hieraus resultiert eine deutliche klinische Nähe, welche insbesondere bei den maßgeschneiderten Formulierungen zu Tage tritt.

Als Vorteil für die klinische Anwendbarkeit gelten u.a. (Albani et al. 2008, S.125-137):

1. Strukturierung klinischen Materials zur Verlaufsbeschreibung therapeutischer Prozesse

2. Leichtes Erlernen der Methode zur klinischen Anwendung, daher nicht nur für erfahrene Kliniker geeignet

3. Durch geringere Komplexität ist die Methode in der klinischen Arbeit entschiedener verankert als zum Beispiel die SASB-Methode (u.a.)

4. Kombinierbarkeit mit verschiedensten Formen der Datenerhebung (Transkripte, Videos, live-Interviews, Stundenprotokolle usw. - Zander et al. 1995).

Als methodenkritische Anmerkungen tragen Albani et al. (2008) u.a. vor:

1. Weitgehend subjektive Beurteilung und Zuordnung zu Kategorien

2. SK und CL nivellieren individuell unterschiedliche Patientenaussagen

3. Bislang unzureichende Berücksichtigung jener Faktoren, welche das Erzählen des Patienten beeinflussen (z.B. Art der Therapie, Erstkontakt oder Folgestunde usw.)

4. Durch die Annahme, alles Häufige sei gleichzeitig zentral, können seltene, aber vielleicht wichtige Muster untergehen. Häufige Aussagen stellen möglicherweise nur die Abwehr eines weniger häufigen Themas dar. Sollte daher auch ein Fokus auf seltenere Themen gerichtet werden?

Für zukünftige Untersuchungen sind u.a. wünschenswert (Albani et al. 2008):

1. Replikationen vorliegender Untersuchungen an größeren Stichproben

2. Ermittlung diagnosespezifischer Beziehungsmuster

3. Weitere Untersuchungen zur Veränderung von Beziehungsmustern durch Psychotherapie (Beginn, Verlauf, Ende) in verschiedenen Behandlungsformen

4. Untersuchungen zum Einfluss des Therapeuten auf das, was Patienten berichten

5. Untersuchung des Nutzens der Methode für klinische Ausbildung

6. Weitere Untersuchungen zur Validität der Methode. 
Die vorliegende Dissertation geht auf einige der von Albani et al. (2008) genannten Herausforderungen ein:

1. Sie untersucht eine ausreichend große Stichprobe einer diagnostisch homogenen Patientengruppe

2. Da alle Patienten die Hauptdiagnose GAS tragen, leisten unsere Untersuchungen einen Beitrag zur Ermittlung diagnosespezifischer Beziehungsmuster

3. Sie untersucht Beziehungsmuster sowohl zu Beginn der Therapie, als auch am Ende, sodass Veränderungen im Therapieverlauf aufgezeigt werden können. Was ist zentral am Anfang und was am Ende? Was verändert sich? Welche Schlussfolgerungen können daraus gezogen werden?

4. Zudem erfolgt ein Vergleich verhaltenstherapeutisch und psychodynamisch behandelter Patienten. Die Ergebnisse tragen zur Beantwortung der Frage bei, welchen Einfluss die Behandlungsform auf das Erzählte hat. Weisen Patienten mit derselben Grunderkrankung in Abhängigkeit vom Therapieverfahren unterschiedliche zentrale Themen auf?

Der folgende Abschnitt befasst sich mit der Diskussion der speziellen Fragestellungen. Zum einen soll noch einmal allgemein auf die Reliabilität des ZBKT-Verfahrens und dessen Anwendbarkeit im klinischen Alltag eingegangen werden, zum anderen werden einige der Forderungen Albani`s hinsichtlich zukünftiger ZBKT-Forschung bearbeitet. Gibt es ein spezifisches ZBKT angstgestörter Patienten? Ist es mit dem ZBKT anderer Patientengruppen vergleichbar? Unterscheiden sich die ZBKT`s von verhaltenstherapeutisch und psychodynamisch therapierten GAS-Patienten? Wie verändern sie sich im Therapieverlauf? Unterscheiden sich auch die maßgeschneiderten ZBKT`s von VT- und SET-Patienten? 


\subsection{Diskussion der Frage 1}

Kann aus dem direkten Vergleich der durch verschiedene Kategoriensysteme bestimmten Inter-Rater-Reliabilität (IRR) auf Clusterebene eine Tendenz zur Bevorzugung eines Kategoriensystems zur Bestimmung des ZBKT's abgelesen werden? Darf eine relativ kurze Schulungsdauer zum Erlernen der ZBKT-Methode als ausreichend eingestuft werden?

Die älteste ZBKT-Reliabilitätsstudie geht weit in die siebziger Jahre des 20. Jahrhunderts zurück (Luborsky 1977). Sie zeigt moderate Übereinstimmungen. Luborsky und CritsChristoph (1998, S. 102-103) präsentieren eine Auswahl von Reliabilitätsstudien der darauf folgenden 20 Jahre (Crits-Christoph et al. 1988; Popp et al. 1996; Barber et al. 1995). Die meisten dieser Studien weisen sehr gute Übereinstimmungsraten auf.

Auch in dieser Arbeit werden sehr gute IRR erreicht. Allerdings beziehen sich - im Gegensatz $\mathrm{zu}$ den vorliegenden Untersuchungen - nicht alle der genannten Studien auf BE`s aus Therapiesitzungen, sondern z.B. auch auf BE`s aus Träumen und RAP-Interviews (Relationship Anecdote Paradigm) (Luborsky 1978). Die Ergebnisse von Reliabilitätsstudien, welche - vergleichbar mit unseren Untersuchungen - BE`s aus Therapiesitzungen untersuchten, sind mit unseren Ergebnissen aber durchaus vergleichbar.

Generell sind die Rahmenbedingungen zur Ermittlung der IRR zu berücksichtigen. Während in den genannten Studien die klassischen ZBKT-Cluster verwendet wurden, schließt die vorliegende Arbeit auch die später entwickelten Clustersysteme von Körner et al. (2000) und Albani et al. (2002 a,b) ein und stellt den wichtigen Vergleich her. Dieser Vergleich gibt keinen Hinweis darauf, eines der Auswertungsverfahren aus methodischen Gründen zu bevorzugen, wenn auch die LU-Cluster (vereinfachte Version) zusammenfassend diskret höhere Übereinstimmungswerte liefern. Sie scheinen die Reliabilität also zu verbessern, obwohl sie 13 Cluster (A-M) im Vergleich zu den 8 klassischen Barber-Clustern und 8-10 alternativen Körner-Clustern bereit halten. Aufgrund der höheren Anzahl an Clustern hätte eine geringere IRR aufgrund höherer Streuung nicht überrascht. Allerdings wurden die Ergebnisse nicht auf Signifikanz getestet. Es liegt ein rein deskriptiver Vergleich vor.

Da die ,vereinfachte Version“ LU(v) des LU-Auswertungsverfahrens und nicht die originale Version LU(o) mit allen Subkategorien verwendet wurde, sind die Ergebnisse vor diesem Hintergrund einzuordnen.

Die unterschiedlichen Vorgehensweisen zur Bestimmung des ZBKT`s mit verschiedenen Auswertungsverfahren zeigen verschieden stereotype Muster auf. Mit den „neueren“ Verfahren lassen sich differenziertere Muster erkennen. Die durch Albani (2002 a,b) etablierte Unterscheidung in subjekt/objektbezogene Wünsche und positive/negative Reaktionen scheint diesbezüglich von Bedeutung zu sein.

Wir können mit unseren Untersuchungen nicht zeigen, dass die Körner-Cluster die Reliabilität gegenüber den klassischen Barber-Clustern steigern, Körner et al. (2002) bestätigen unter Verwendung der alternativen Clusterlösung allerdings die Verbesserung der klinischen Validität der Methode.

Die IRR wurde zwischen zwei Ratern bestimmt. Ein Aspekt, der zukünftig weiter untersucht werden könnte, ist der der Einflussnahme der Gleich- oder Gemischtgeschlechtlichkeit der Rater-Paare. Im vorliegenden Patientenkollektiv erzielten die gemischt geschlechtlichen Rater-Paare höhere durchschnittliche Übereinstimmungen als das gleichgeschlechtliche RaterPaar. An größeren Fallzahlen könnte geprüft werden, ob sich anhand von 
Geschlechterkombination, Alter oder Ausbildungsstand eines Rater-Paares signifikante Unterschiede in der Höhe der IRR dokumentieren lassen.

Da die Rater als Studenten ohne Vorkenntnisse an die ZBKT-Methode herangeführt wurden und dennoch mit allen Kategoriensystemen ausreichend hohe IRR erreichten, kann von einer soliden Vorbereitung und Schulung ausgegangen werden. Die Methode scheint auch bei einer relativ kurzen Schulungsdauer für Laien gut erlernbar zu sein. In klinischer Erfahrung und psychodynamischer Orientierung sehen Albani et al. (2008, S. 195) einen Vorteil, jedoch keine Voraussetzung für gute IRR. Entscheidend sei „systematisches Üben“.

Fazit:

Mit allen Kategoriensystemen wurden sehr gute IRR erreicht. Aus methodischen Gründen kann keine Empfehlung zur Bevorzugung eines Kategoriensystems ausgesprochen werden. Allerdings wurden die Ergebnisse nicht auf Signifikanz getestet. Mit den LU-Clustern (Albani et al. 2002 a,b) lassen sich differenziertere Muster aufzeigen, weshalb sie für den klinischen Alltag bevorzugt verwendet werden sollten. Die Vorbereitung und Schulung der Rater darf als ausreichend eingestuft werden, die ZBKT-Methode ist auch für Laien innerhalb einer relativ kurzen Schulungsdauer gut erlernbar, ohne dass Defizite hinsichtlich der Höhe der IRR befürchtet werden müssten. Unsere Ergebnisse sind mit denen anderer Reliabilitätsstudien vergleichbar. 


\subsection{Diskussion der Frage 2}

Gibt es ein spezifisches ZBKT angstgestörter Patienten? Lässt es sich mit dem anderer Patientengruppen vergleichen?

Grundsätzlich ist zu berücksichtigen, dass zum Untersuchungszeitpunkt nicht alle Patienten (lediglich 15 von 45) ausschließlich an GAS litten. Da der größere Teil der Patienten komorbide Störungen aufwiesen, musste zunächst geklärt werden, ob unsere Untersuchungen überhaupt dazu beitragen können, ein spezifisches Thema angstgestörter Patienten zu finden, oder ob die Komorbiditäten das Spezifische des Themas verzerren.

Da es sich bei der GAS im Sinne der Hauptdiagnose definitionsgemäß um die schwerste mentale Störung der Patienten handelt und komorbide Störungen ein häufiges Phänomen darstellen, welches vermutlich nur unter Verlust der Validität der Aussagen gänzlich ausgeschlossen werden könnte, entschieden wir uns, das Vorliegen von Komorbiditäten zu vernachlässigen. Zu den häufigsten komorbiden Störungen des Patientenkollektives zählen andere Angststörungen, depressive Störungen, komorbide Anpassungsstörungen, Zwangsstörungen und somatoforme Störungen.

Betrachtet man die häufigsten $\mathrm{W}$ unseres Patientenkollektives, so fällt auf, dass die klassischen ZBKT-Cluster (Barber et al. 1990) eine sehr breite Verteilung ohne signifikantes Herausragen einer oder mehrerer häufiger Cluster (CL) zeigen. Ein zentraler Wunsch lässt sich im AV nicht ablesen. Anders verhält es sich bei den LU-CL (Albani et al. 2002 a,b), die sowohl in der WO- als auch in der WS-Komponente eine deutliche Betonung der harmonischen Sequenzen aufzeigen, innerhalb derer sich häufigste CL deutlicher demarkieren.

Die Differenzierung der $\mathrm{W}$ in subjekt- und objektbezogene, sowie in harmonische und disharmonische scheint das Erkennen häufigster und somit vermutlich zentraler Wünsche im AV zu erleichtern, obwohl für das LU-System 13 Cluster und somit 5 Cluster mehr als im Barber-System vorliegen. Auch wenn sich zentrale Wünsche mit den LU-CL scheinbar leichter ablesen lassen, so stehen ihnen die Barber-Cluster bezüglich der Höhe der IRR fast in nichts nach.

Mit der Frage nach dem spezifischen Thema angstgestörter Patienten wird versucht, ein den GAS-Patienten eigenes Muster zu finden, welches unspezifisch durch die Methode oder durch das psychotherapeutische Setting hervorgerufen wird. Was eint diese Patienten? Was unterscheidet sie von anderen Patientengruppen?

Die Theorie vom „steuernden Objekt“ (1986/2000) besagt: „Allen Patienten mit Angstsymptomatik ist ein spezifischer Umgang mit Objekten gemein: ihr Umgang mit Menschen und Dingen, die ihnen Menschen ersetzen, wird durch die Suche nach steuernden Funktionen der Objekte gekennzeichnet. ... Als Ersatz für das mangelhaft ausgebildete innere steuernde Objekt werden in der Außenwelt steuernde Ersatzobjekte gesucht...Dem steuernden Objekt sind Ich-Funktionen, welche der Kompetenz einer sozial adäquaten Realitätsbewältigung dienen, zugeordnet".

Wir nehmen an, dass unter allen Angstpatienten auch die GAS-Patienten steuernde Funktionen suchen und sie ihrem Therapeuten zuordnen möchten. Belegende Hinweise sehen wir in den W-CL. Die häufigsten LU-CL lassen die Interpretation, es liege der Wunsch nach einem steuernden Objekten vor, durchaus zu: ,,andere sollen mich unterstützen, souverän sein, mich lieben und sich mir zuwenden“. Die häufigsten klassischen W-Cluster ,gut und 
wohl fühlen, Erfolg und Leistung erreichen " lassen diese Interpretation hingegen nur indirekt zu. Sie wirken eher verallgemeinernd, der Wunschcluster ,kontrolliert und verletzt werden und keine Verantwortung haben " gar verzerrt, weshalb wir die im Cluster enthaltenen SK einsehen: „Hilfe, Unterstützung bekommen, sich mit dem anderen identifizieren, dem anderen ähnlich sein, verletzt und bestraft werden, passiv, unterwürfig und abhängig sein". Die SK lassen Parallelen zur offensichtlicheren Formulierung der LU-CL erkennen und könnten als Wunsch nach steuernder Funktion von Objekten verstanden werden, welcher das Vermeiden eigener, aktiver Impulse vorbereitet. Dass auf die Ebene der SK zurückgegangen werden muss, werten wir als Unzulänglichkeit der klassischen ZBKT-CL.

Nach Crits Christoph et al. (1995) zeigen GAS-Patienten häufig frühkindlich, präödipale Wünsche (z.B. ,Ich möchte jemanden haben, der immer für mich da ist"). Auch hierin lässt sich der Wunsch nach einem steuernden Objekt erkennen.

Die DSM-IV-Klassifikation stellt „unkontrollierbare Sorgen“ als Hauptmerkmal von GASPatienten in den Mittelpunkt. Im Wunschverhalten des untersuchten Patientenkollektives lassen sich Hinweise finden, welche für das Vorliegen von Sorgen sprechen könnten (z.B. „möchte Hilfe, Unterstützung, möchte abhängig sein, beschützt werden“).

Die häufige LU-WS-Komponente ,, souverän sein, zurückziehen können“ darf als konflikthaft erlebte Wechselbeziehung verstanden werden, welche einen gewissen Vermeidungsaspekt erkennen lässt, der ebenfalls vielen GAS-Patienten gemein ist.

Zusammenfassend stellen unter anderem die Suche nach steuernden Objekten, unkontrollierbare Sorgen und Vermeidungswünsche Gemeinsamkeiten von GAS-Patienten dar, welche an unserem Patientenkollektiv durch LU-CL und bedingt auch durch klassische CL sichtbar gemacht werden können. Dass bei den klassischen CL auf die Ebene der SK zurückgegriffen werden muss, sehen wir als Defizit dieser Clusterstrukturen an.

Ob diese Gemeinsamkeiten als GAS-spezifisch gewertet werden können, ist allerdings fraglich, da sie sicherlich auch auf andere Gruppen angstgestörter Patienten zutreffen und nicht zwingend durch das therapeutische Setting oder die Methode bedingt sein müssen. Auch sehen wir individuelle Lebensverläufe als maßgeblich prägendes Element von ZBKT`s an, weshalb diese nicht für eine ganze Patientengruppe vereinheitlicht werden können. Mit dieser Schlussfolgerung schließen wir uns Crits Christoph et al. (1995) an.

Für den Vergleich der Wunschkomponente von GAS-Patienten mit der Wunschkomponente anderer Patientengruppen ziehen wir u.a. die Beziehungsmuster einer 31-jährigen italienischen Patientin mit der Diagnose „Angststörung mit Somatisierung und Depression“ heran. Ihr ZBKT wurde mit einer italienischen Version der LU-CL (Vicari et al. 2003/2008) bestimmt, die Patientin befand sich zum Untersuchungszeitpunkt in einer verhaltenstherapeutischen Kurzzeittherapie (Sacchi 2005, vgl. Albani et al. 2008). Das Beispiel wurde herangezogen, da das Störungsbild hinsichtlich der Komorbiditäten in etwa unserem Patientenkollektiv entspricht. Die zentralen Wunschthemen dieser Patientin waren „Zuwendung “ und ,eigene Souveränität“.

Vanheule et al. (2006) untersuchten, welche Kategorien des ZBKT`s in Zusammenhang mit depressiven Symptomen stehen. Die häufigsten Wünsche depressiver Patienten waren ,sich glücklich fühlen“, ,, verstanden werden“, ,, der Favorit sein“, ,,geholfen werden “ und „,gut sein".

Chance et al. (2000) bestimmten ZBKT's bei Patienten mit BorderlinePersönlichkeitsstörungen (mit und ohne Suizid). Die zentralen Wünsche dieser Patienten waren ,geliebt und verstanden werden“. Drapeau und Perry (2004) untersuchten ebenfalls 
Patienten mit Borderline-Persönlichkeitsstörungen. Sie fanden „Unterstützung durch andere ", ,, beachtet werden ", ,, sich zurückziehen “, ,, selbstbestimmt und souverän sein" als häufigste Wunschkategorien.

Alle genannten Patientengruppen weisen ein mit unserem Patientenkollektiv vergleichbares Wunschverhalten auf. Bezüglich der Reproduzierbarkeit eines GAS-typischen Wunschverhaltens sind wir daher skeptisch und hinterfragen die vorliegenden Ergebnisse kritisch. Dass derart unterschiedliche Patientengruppen ein verblüffend ähnliches Wunschverhalten aufweisen, wirft die Frage auf, warum Wunschkomponenten verschiedenster Patientengruppen auf dieselben ZBKT-CL eingeordnet werden.

Nun bleibt einerseits anzunehmen, dass die immer gleichen Wünsche als Artefakt der ZBKTMethode zu verstehen sind, andererseits darf vermutet werden, dass sie den Umgebungsfaktoren geschuldet sind, wie es zum Beispiel das therapeutische Setting darstellt.

Uhmann et al. (2010) untersuchten die Spezifität interpersoneller Probleme von GASPatienten und verglichen sie mit der anderer Patientengruppen (Patienten mit anderen Angststörungen und Depressionen). Sie fanden keine klinisch diagnostische Spezifität für GAS-Patienten. Der Vergleich darf hinsichtlich der miteinander verglichenen diagnostischen Gruppen hier angeführt werden, muss allerdings vorsichtig diskutiert werden, da ZBKT`s nicht unmittelbar mit interpersonellen Problemen verglichen werden können. Es kann daraus jedoch eine Idee für künftige Untersuchungen abgeleitet werden, welche den Einfluss der umgebenden Faktoren untersuchen, wie es z.B. das psychotherapeutische Setting darstellt.

Die RO-Komponente zeigt unabhängig von Patientengruppe und Auswertungssystem „Zurückweisung“ und „Unzuverlässigkeit“ als häufigste CL auf. Im Wortlaut der klassischen ZBKT-CL: ,, andere weisen mich zurück und sind gegen mich “, im Wortlaut der LU-CL: „, andere sind unzuverlässig und weisen mich zurück“. Die verschiedenen Patientengruppen unterscheiden sich hierbei nicht, was als Artefakt beider Auswertungssysteme verstanden werden kann. Barber et al. (1995) schlagen eine Erklärung mit klinischem Hintergrund vor. So würden depressive Patienten dazu neigen, das Verhalten Anderer als ablehnend zu werten, sich zudem mehr mit ablehnenden Personen umgeben oder gar andere dazu veranlassen, ablehnend $\mathrm{zu}$ handeln. Jedoch zeigen auch alle anderen Patientengruppen das immer gleiche Clusterverhalten in der RO-Komponente auf, was die Erklärung entkräftet.

Die RS-Komponente variiert im GAS-Patientenkollektiv unserer Untersuchungen zwischen „,Enttäuschung“, „Depression“, „Angst“ und „,̈rger“. Im Wortlaut der klassischen ZBKT$\mathrm{CL}$,Ich bin enttäuscht und deprimiert“, im Wortlaut der LU-CL ,, Ich bin unzufrieden, habe Angst und bin verärgert, fühle mich aber auch wohl".

Während die klassischen CL wie erwartet die üblich monotonen RS-Muster aufzeigen, können die LU-CL differenziertere Reaktionen der Subjekte herausfiltern. Dies wird als qualitativer Zugewinn der LU-CL gewertet, welcher auch durch eine diskret höhere IRR belegt wird.

Verglichen mit unserem Patientenkollektiv sind die häufigsten RS-Komponenten der italienischen Patientin ,,unzufrieden " und ,fremdbestimmt". Depressive Patienten (Vanheule et al. 2006) ,,mögen andere nicht", sind ,,hilflos “, ,,mögen andere doch“, haben sich ,,unter Kontrolle" und sind „selbstzerstörerisch“. Borderline-Patienten sind ,depressiv" und „,enttäuscht" (Chance et al. 2000), sowie ,,unzufrieden“, ,,verängstigt", ,,fremdbestimmt“, „, abhängig “ und ,schwach“ (Drapeau und Perry 2004). 


\section{Fazit:}

Während die W- und RO-Komponenten ungeachtet der Patientengruppe große Ähnlichkeiten aufweisen, zeigt die RS-Komponente verschiedene Facetten, was für eine gute klinische Validität der ZBKT-Methode in dieser Kategorie spricht. Ob allein das Kriterium der Verschiedenheit und guten klinischen Validität ausreicht, um die RS-Komponente als GASspezifisch zu werten, bedarf weiterer Untersuchungen. Insbesondere müssen die Ergebnisse vor dem Hintergrund der rein deskriptiven Statistik eingeordnet werden.

Wir gehen zusammenfassend aktuell nicht davon aus, dass sich mit Hilfe der ZBKT-Methode spezifische ZBKT`s angstgestörter Patienten erkennen lassen und schließen uns den Ausführungen Crits Christoph et al. (1995) an, die mit ihren Untersuchungen zu dem selben Schluss kamen. Aufgrund der wesentlichen Ähnlichkeit zu den CL anderer Patienten sind wir mit der Interpretation, das ZBKT sei GAS-typisch zurückhaltend. Weitere, ähnlich aufgebaute Untersuchungen müssen folgen, um der Frage nachzugehen, ob - und wenn ja, inwiefern die ZBKT-Methode im Bereich der W- und RO-Komponente einen methodischen Fehler aufweist und welche Rolle unspezifische Umgebungsfaktoren, wie das psychotherapeutische Setting, spielen. Sind diese Fragen geklärt, ist der Blick auf zentrale Themen von GASPatienten möglicherweise freier.

Unabhängig davon halten wir die Suche nach steuernden Objekten, ein gewisses Vermeidungsverhalten und unkontrollierbare Sogen für GAS-typisch, wenn auch nicht spezifisch. Hinweise für diese Gemeinsamkeiten lassen sich aus den häufigsten CL ablesen. Die Differenzierung der LU-W-CL in subjekt- und objektbezogene, sowie die Unterscheidung in harmonische und disharmonische Sequenzen erleichtert das Erkennen häufigster, zentraler Wünsche und klinisch bedeutsamer Inhalte (z.B. Beziehungswunsch). Mit den klassischen ZBKT-CL lassen sich häufigste Wünsche weniger deutlich erkennen. Dies korreliert mit der Höhe der IRR, welche für die LU-CL in der Wunsch-Kategorie am größten ist. 


\subsection{Diskussion der Frage 3}

Gibt es hinsichtlich zentraler Themen Unterschiede zwischen verhaltenstherapeutisch und psychodynamisch-tiefenpsychologisch behandelten GAS-Patienten?

ZBKT`s von GAS-Patienten wurden hauptsächlich im Rahmen von SET-Sitzungen bestimmt. Die dritte Fragestellung soll zur Klärung der Frage beitragen, ob sich unterschiedliche ZBKT`s in Abhängigkeit von der Art des Therapieverfahrens dokumentieren lassen. Hierzu erfolgt der direkte, deskriptive Vergleich der ZBKT`s von SET- und VT-Patienten im AV. Die Ergebnisse der klassischen und LU-CL werden miteinander verglichen.

Bei VT-Patienten bilden sich die klassischen ZBKT-CL 7 und 8 (,Erfolg Leistung erreichen “, ,gut und wohl fühlen") häufiger ab als andere Kategorien, woraus wir ableiten, dass sich VT-Patienten mit bewältigungsorientierten Themen und Situationsbezogenheit kritisch auseinandersetzen, während Beziehungswünsche weniger zentral sind.

Verglichen mit den häufigsten klassischen CL von SET-Patienten (CL 5, 3, und 4: ,anderen nahe sein“, ,kontrolliert und verletzt werden“, „Abstand haben, zurückziehen“), welche deutliche Beziehungswünsche für diese Patientengruppe erkennen lassen, handelt es sich bei den Wünschen der VT-Patienten möglicherweise um die bewusstseinsnäheren, sozial akzeptierten und hierdurch naheliegenden Wünsche.

Die klassischen ZBKT-CL können deutliche Unterschiede zwischen den beiden Patientengruppen sichtbar machen (Beziehungswunsch vs Situationsbewältigung).

Im Gegensatz dazu sind die häufigsten LU-W-CL von SET- und VT- Patienten weitestgehend identisch. Harmonische Beziehungswünsche (WO: „Unterstützung, Souveränität Liebe, Zuwendung") und Wünsche nach Selbstbestimmtheit (WS: „souverän sein, lieben, wohlfühlen, zurückziehen ") gehören für beide Patientengruppen zu den häufigsten Wünschen, woraus wir ein konflikthaftes und bewusstes Erleben dieser Themenbereiche ableiten. Der Wunsch nach eigener Souveränität (LU WS: D) ist bei VT-Patienten noch ausgeprägter, was durch die LU-eigene Differenzierung der Wünsche in subjekt- und objektbezogene sichtbar gemacht werden kann.

Während die klassischen ZBKT-CL Hinweise darauf geben, dass an der selben Grunderkrankung leidende Patienten in Abhängigkeit von einer zufälligen Zuteilung zu einer Therapiegruppe unterschiedliche, therapietypische zentrale Wunschthemen (Beziehungswunsch vs Situationsbewältigung) aufweisen, scheint sich das mit den LU-CL erfasste Wunschverhalten von VT- und SET-Patienten nicht zu unterscheiden.

Wir diskutieren daher die Möglichkeit des Vorliegens eines Fehlers im LU-System gegenüber einer Eigenheit der klassischen CL, die uns fälschlicherweise zur Annahme unterschiedlicher W-CL für VT- und SET-Patienten führt.

Der Theorie vom „steuernden Objekt“ (König 1986/2000) zufolge könnte angenommen werden, dass GAS-Patienten ihrem Therapeuten gerne steuernde Funktionen zuschreiben möchten und somit für wichtig halten, was er für wichtig hält. Diese Theorie würde den Ergebnissen der klassischen ZBKT-CL entsprechen. Sollten VT- und SET-Patienten mit derselben Grunderkrankung tatsächlich in Abhängigkeit von Therapieverfahren und Therapeut unterschiedliche Wunschthemen aufweisen, so dürften sich diese Beobachtungen reproduzieren lassen. Weitere Untersuchungen sollten folgen. Insbesondere muss genauer untersucht werden, inwiefern Vorgespräch und dem Untersuchungszeitpunkt vorausgegangene Therapiestunden Einfluss auf das ausüben, was gesagt wird. 
Unsere Untersuchungen zeigen auch, dass die Unterschiede zwischen VT- und SET-Patienten bereits zum Zeitpunkt des ersten Etappen-ZBKT`s der dritten und fünften Therapiestunde bestehen. Um den Zeitpunkt des Eintretens der Unterschiede zwischen VT- und SETPateinten genauer zu eruieren, sollten Verlaufs- und Etappen-ZBKT`s in kürzeren Abständen erfolgen.

Die Reaktionen der Objekte sind unabhängig von der Therapiegruppe entsprechend der Formulierung klassischer ZBKT-CL - hauptsächlich ,,zurückweisend“ und „,unzuverlässig“. Diese CL sind allen anderen an Häufigkeit weitaus überlegen. Nahezu alle Studien zum ZBKT zeigen dieses Clusterverhalten unabhängig von Therapiegruppe oder Grunderkrankung der Patienten (z.B. Vanheule et al. 2006, Drapeau und Perry 2004, Chance et al. 2000).

Dass alle Patienten ungeachtet ihrer unterschiedlichen Grunderkrankungen und auch sonstigen, z.B. zwischenmenschlichen Konstellationen und Umstände die immer gleichen Reaktionen bei anderen hervorrufen, muss als Schwachstelle der klassischen CL gewertet werden. Zwar zeigen auch die LU-CL bei beiden Patientengruppen dieselben häufigsten ROCL (,,unzuverlässig“, „,zurückweisend“), allerdings besteht eine breitere Verteilung über alle CL hinweg, was für eine bessere klinische Anwendbarkeit der LU-CL spricht. Sie zeigen zum Beispiel, dass sich VT Patienten - im Gegensatz zu SET-Patienten - von anderen zwar mehr „zurückgewiesen“ fühlen, diese aber als „weniger dominant“ erleben. Entsprechend andersherum verhält es sich bei SET-Patienten. Das Erkennen solcher Feinheiten wird deutlich der Klarheit und Qualität der LU-CL zugeschrieben.

Während GAS-Patienten, wie auch andere Patientengruppen (z.B. Vanheule et al. 2006, Drapeau und Perry 2004, Chance et al. 2000), entsprechend der Formulierung klassischer CL vorrangig den RS-CL 7 ,enttäuscht“ und „,deprimiert" aufweisen und dieser CL in beiden Therapiegruppen eindeutig als häufigster CL zu erkennen ist, zeigen die LU-CL mehrere gleichhäufige CL und somit eine homogene Verteilung mit mehreren Facetten des Reaktionsverhaltens. Neben „Unzufriedenheit“, „Angst" und „Ärger" sticht auch eine häufige positive Reaktion ins Auge: ,lieben und wohl fühlen“. VT und SET Patienten unterscheiden sich auch bei den LU-CL nicht. Da die klassischen CL in mehreren Studien an verschiedenen Patientengruppen nachweislich zumeist CL 7 als häufigste Reaktion zeigen, liegt auch hier die Frage nach der strukturellen Schwäche der klassischen ZBKT-CL nahe.

\section{Fazit:}

Zusammenfassend ist festzuhalten, dass VT und SET Patienten unterschiedliche, therapietypische (Beziehungswunsch vs. Situationsbewältigung) zentrale Wunsch-Themen aufweisen, wenn das ZBKT mit den klassischen ZBKT-CL bestimmt wird. Es zeigen sich keine Unterschiede, wenn das ZBKT mit den LU-CL bestimmt wird.

Wir gehen entweder von einem Artefakt des LU-Systems oder der klassischen CL im Wunschbereich aus, tendieren aber dazu, die Verschiedenheit der Wunschthemen von SETund VT-Patienten anzuerkennen, was wir mit der Theorie vom steuernden Objekt (1986/2000) begründen. Patienten tendieren dazu, für wichtig zu halten, was auch ihre Therapeuten für wichtig halten. Wir gehen davon aus, dass die Behandlungsmethode wesentlichen Einfluss auf das Erzählen des Patienten hat. Was Therapeuten und Patienten für wichtig halten scheint in Ansätzen deckungsgleich. Weitere Untersuchungen an ähnlichen Stichproben müssen folgen, um die Reproduzierbarkeit der Ergebnisse zu prüfen. Was bedeutet die Ähnlichkeit von Patienten- und Therapeutenaussagen für die klinische Arbeit? 
Wie entsteht sie? Ist das Wunschverhalten anderer Patientengruppen und ihrer Therapeuten auch ähnlich?

Aufgrund der eindrucksvollen Unterschiede zwischen den klassischen CL und LU-CL ist davon auszugehen, dass auch das Verfahren zur Bestimmung des ZBKT`s dieses letztlich prägt.

Die häufigsten Reaktionskomponenten von VT- und SET-Patienten unterscheiden sich nicht. Allerdings zeigen die LU-CL eine wesentlich breitere Verteilung über mehrere CL, was sie differenzierter wirken lässt, ein homogeneres Bild aufzeigt und für die strukturelle Stärke der CL spricht. Die Ergebnisse der klassischen CL weisen darauf hin, dass ein systematischer Fehler im Erfassen häufigster Kategorien vorliegen könnte, da verschiedene Patienten ungeachtet ihrer Grunderkrankung konsequent auf den häufigsten RO-CL 5 und den RS-CL 7 eingeordnet werden. 


\subsection{Diskussion der Frage 4}

Verändern sich zentrale Themen von VT- und SET-Patienten während des Therapieverlaufes?

Auch die Beobachtung der Veränderung zentraler Themen von VT- und SET-Patienten erfolgte deskriptiv. Die Ergebnisse sollten daher vorsichtig interpretiert werden, können aber Anhalt dafür geben, welche Hypothesen in zukünftigen Studien auf Signifikanz geprüft werden können.

Luborsky verglich durch Psychotherapie gebesserte und weniger gebesserte Patienten und stellte fest, dass das ZBKT am Anfang und am Ende der Therapie gut zu erkennen war. Die weniger gebesserten Patienten wiesen eine größere zeitliche Stabilität des ZBKT`s auf. Insgesamt blieb die W-Komponente stabiler als die Reaktionskomponenten (Luborsky 1999, S. 167). Die gebesserten Patienten konnten am Therapieende wesentlich besser mit ihren Beziehungsproblemen umgehen, da ihnen umfangreichere Bewältigungsmöglichkeiten (Luborsky 1999, S. 114) zur Verfügung standen. Auch Wiegand (2010) konnte eine Zunahme der Handlungs- und Bewältigungsmöglichkeiten bei GAS-Patienten beobachten.

Aus dem Vergleich von VT- und SET Patienten erhoffen wir uns Aufschluss darüber, ob und wie sich die ZBKT`s der beiden Behandlungsgruppen entwickeln. Erneut werden die durch klassische und LU-CL erfassten Veränderungen verglichen und diskutiert.

\section{Wünsche-SET:}

Zum Zeitpunkt T1 sind Vermeidungswünsche (klassischer CL 4 „Auseinandersetzungen vermeiden") und Beziehungswünsche (klassischer CL 5, 6 ,geliebt und verstanden werden, anderen nahe sein") bei SET-Patienten wesentlich häufiger als andere Wünsche. Wir werten dies als Hinweis dafür, wie konflikthaft Vermeidung und Beziehung erlebt werden. Wiegand (2010) stellte im Rahmen der Auswertung einer Selbstbeurteilung von GAS-Patienten fest, dass SET-Patienten den Wunsch ,,Ruhe und Gelassenheit “ häufiger nennen als VT-Patienten, was wir als deckungsgleich zu unseren Untersuchungen einstufen.

Zum Zeitpunkt T2 haben Vermeidungs- und Beziehungswünsche an Häufigkeit verloren, die klassischen CL 3 und 2 ,kontrolliert und verletzt werden, mich anderen widersetzen, andere verletzen") sind jetzt deutlich stärker. Wir interpretieren diese Veränderung als Beginn der Auseinandersetzung mit konfliktbehafteten Themen, welche möglicherweise nun eine bewusstseinsnähere Ebene erreicht haben. Entsprechend belegt wird dies mit der Abnahme des CL 4 (,Auseinandersetzung vermeiden“). Auch die Beobachtungen Wiegands (2010) zeigen, dass SET-Patienten Probleme als greifbarer und insgesamt seltener vorkommend bewerten und ihnen mehr Handlungs- und Bewältigungsmöglichkeiten zur Verfügung stehen.

Mit den LU-CL wird zu Therapiebeginn und -ende ein ähnliches Verteilungsmuster der WOCL mit Betonung der harmonischen Sequenzen bei SET-Patienten nachgewiesen. Beziehungs- und Bindungswünsche bleiben häufig bestehen und nehmen sogar noch etwas zu, während CL M ,,sich zurückziehen “ an Häufigkeit verliert. Diese Entwicklung erlaubt den Schluss, dass Rückzug und Vermeidung nicht mehr zu den dominierenden Themen der SETPatienten gehören und daher weniger häufig genannt werden. Im Umkehrschluss könnte es auch ein Indiz dafür sein, dass SET-Patienten sich ihren Konflikten und ihrem Gegenüber stellen, was wiederum auch die Zunahme der Häufigkeit von Beziehungs- und Bindungswünschen erklären würde.

Auch die WS-CL zeigen zum Zeitpunkt T1 und T2 ein ähnliches Verteilungsmuster. Deutlich häufiger wird allerdings CL K ,dominieren“, während CL M „,sich zurückziehen“ 
eindrucksvoll an Häufigkeit verliert. Hier zeigt sich ebenfalls, wie Tendenzen zu einer aktiveren Auseinandersetzung mit problembehafteten Themen entstehen, welche nun wesentlich mehr Raum einnehmen. Dominanzwünsche scheinen bewusstseinsnäher erlebt zu werden.

\section{Wünsche-VT:}

Bei VT-Patienten sind zum Zeitpunkt T1 die klassischen CL 7, 8 und 3 (,gut und wohl fühlen, Erfolg und Leistung erreichen, keine Verantwortung haben") etwas stärker als andere CL. Bewältigungsorientierung im Alltag gehört zu den naheliegenden und im Vordergrund stehenden Themen. Die Selbsteinschätzung der GAS-Patienten (Wiegand 2010) wies wesentlich mehr Angst bei VT-Patienten nach, was über das ZBKT nur selten direkt erfasst wird.

Zum Zeitpunkt T2 sind die CL 7, 8 und 3 noch immer am Häufigsten, allerdings wird insgesamt ein Rückgang der Nennungshäufigkeit (vor allem CL 7 ,,gut und wohl fühlen " und CL 3 , kontrolliert und verletzt werden, keine Verantwortung haben “) verzeichnet, was zu einer breiteren Verteilung auf alle CL führt und das Muster dadurch weniger starr wirkt. Die nachlassende Häufigkeit der Nennung dieser CL legt den Schluss nahe, den VT-Patienten falle die Situationsbewältigung nicht mehr so schwer, weshalb sie weniger Energie darauf verwenden und sie allmählich aus dem Bewusstsein und somit aus dem Fokus des Berichtens schwindet. All dies könnte für die Entwicklung von Veränderungen sprechen, welche zum Aufbrechen starrer Muster beitragen. Optisch macht sich das in der beschriebenen breiteren Clusterverteilung bemerkbar. Beziehungsassoziierte Themen nehmen am Ende der Therapie mehr Raum ein. Der CL ,geliebt und verstanden werden " verzeichnet den stärksten Zuwachs. Die CL 2 und 3 verhalten sich gegenläufig (,Widerstand und Kontrolle“ zunehmend, ,, kontrolliert und verletzt werden" abnehmend).

Innerhalb der LU-CL zeigen VT-Patienten zu Therapiebeginn und - ende überwiegend harmonische Wünsche (WO und WS). Die häufigsten CL bleiben in sich bestehen, ihre Nennungshäufigkeit verändert sich jedoch im Therapieverlauf. Während der Wunsch nach „Souveränität“ anderer bedeutend an Häufigkeit gewinnt, verliert der Wunsch nach ,eigener Souveränität" an Nennungshäufigkeit. Dies könnte ein Hinweis dafür sein, dass sich VTPatienten selbst wesentlich souveräner erleben, oder sie der Souveränität aus anderen Gründen weniger Bedeutung beimessen und das Thema somit aus dem Fokus des Berichtens verschwindet. Andere sollen sich weniger ,,zurückziehen“" und weniger „wohlfühlen“. Auseinandersetzungen werden also begangen, auch wenn das unangenehme Gefühle zur Folge hat. Untermalt wird dies von der Zunahme des Wunsches eigener „Dominanz“. Auch Wiegands (2010) Beobachtungen bei VT-Patienten nahmen diese Veränderungen wahr.

\section{Fazit:}

Die klassischen ZBKT-CL zeigen, dass sich im Wunschverhalten von SET-Patienten Veränderungen ergeben, welche Hinweise für eine stärkere Auseinandersetzung mit - nun bewusstseinsnäheren - konfliktbehafteten Themen vor dem Hintergrund des Schwindens von Vermeidungswünschen geben. Die Veränderungen bei VT-Patienten legen den Schluss nahe, dass die Patienten mit der Situationsbewältigung im Alltag besser zurechtkommen.

Die LU-CL zeigen, dass der WO-CL von SET-Patienten weitestgehend stabil bleibt, während die WS-Komponente Hinweise auf eine zunehmende Bewusstseinsnähe von Dominanzwünschen gibt und gleichzeitig Rückzugswünsche eindrucksvoll an Häufigkeit verlieren. In Kombination entsteht auch für die LU-CL der Eindruck, sie würden die Auseinandersetzung der SET-Patienten mit konfliktbehafteten Themen aufzeigen können. Die 
LU-CL geben Hinweise darauf, dass der Wunsch der VT-Patienten nach eigener Souveränität am Therapieende in Zahlen rückläufig ist. Wir interpretieren dies als Hinweis für die bereits stattgefundene Entwicklung eigener Souveränität im Sinne der Situationsbewältigung im Alltag. Auch bei ihnen sind Dominanzwünsche am Therapieende bewusstseinsnäher.

Zusammenfassend halten wir fest, dass sich das Wunschverhalten beider Patientengruppen im Therapieverlauf diskret verändert. Während das grobe Muster bestehen bleibt wird es weniger deutlich und wirkt weniger starr. Mit dieser Beobachtung schließen wir uns Luborsky et al. (1992) und ebenso den klinischen Beobachtungen Pfeffers (1963/2004) an. Wir interpretieren die Veränderungen überdies als Therapieerfolg, da den Patienten - auch bei erhaltenem ZBKT - nach einer erfolgreichen Therapie bessere Bewältigungsmöglichkeiten zur Verfügung stehen, wie auch Luborsky (1999, S. 114) beobachtete.

Unsere Untersuchungen zeigen, dass VT- und SET-Patienten bereits zum Zeitpunkt T1 ein unterschiedliches Wunschverhalten aufweisen, sich die Themen hinsichtlich ihrer Entwicklung im Therapieverlauf jedoch nicht unterscheiden. Wir schlagen vor, die Ergebnisse in zukünftigen Studien auf Signifikanz zu prüfen.

\section{Reaktionen SET und VT:}

Beide Patientengruppen weisen am Therapiebeginn und am Therapieende denselben häufigsten klassischen RO-CL auf. Die erlebte „Zurückweisung “ durch andere (RO-CL 5) nimmt bei beiden Gruppen im Therapieverlauf etwas zu, was wir dahingehend interpretieren, dass sich beide Patientengruppen in der Therapie damit auseinandersetzen, andere als feindlich zu erleben.

Auch die LU-RO-CL filtern „,Unzuverlässigkeit“ und „Zurückweisung“ als häufigste Reaktionen der Objekte beider Patientengruppen. Die LU-RO-CL weisen jedoch insgesamt eine homogenere Verteilung über mehrere häufige RO-CL auf. Wir werten dies als Indiz für die bessere klinische Einsetzbarkeit der LU-CL. Die LU-RO-Komponente ,sich zurückziehen " nimmt bei SET-Patienten, anders als bei VT-Patienten, im Therapieverlauf zu, was die Theorie untermauert, die SET-Patienten setzten sich mehr damit auseinander, andere als feindlich zu erleben, weshalb der CL häufiger vorkommt.

Der Therapieverlauf zeigt interessanterweise auch, dass die Objekte der VT-Patienten am Therapieende stärker verärgert wahrgenommen werden als die der SET-Patienten. Als Erklärungsversuch könnte das Fokussieren der VT Patienten auf akute Problembewältigung, mehr denn auf Beziehungsproblematiken herangezogen werden.

Beiden Patientengruppen sind am Therapieanfang und am Therapieende der häufigste klassische RS-CL 7 (,,bin enttäuscht und deprimiert“) gemein. Während bei SET-Patienten $\mathrm{zu}$ beiden Zeitpunkten eindeutig CL 7 der häufigste RS-CL ist und sich auch die übrigen CL nur minimal verändern, weisen VT-Patienten zum Zeitpunkt T1 drei ähnlich häufige RS-CL auf. Neben dem häufigsten RS-CL 7 sind dies CL 6 ,fühle mich hilflos “ und 8 ,,bin ängstlich “, welche zum Zeitpunkt T2 wesentlich seltener vorkommen, was wir als vermutlich unbewusste Entwicklung hin zu weniger Hilflosigkeit und Ängstlichkeit durch entsprechende Wahrnehmung neu gewachsener Bewältigungsmöglichkeiten durch VT interpretieren. Unsere klinischen Beobachtungen entsprechen denen Wiegands (2010).

Die gleichzeitig deutliche Zunahme des RS-CL 7 (,,bin enttäuscht und deprimiert “) sehen wir im Sinne der Annahme und Auseinandersetzung mit erlebter Enttäuschung. Das Subjekt fühlt sich gleichzeitig aber auch stärker respektiert und akzeptiert, woraus abgeleitet wird, dass es mit Enttäuschung besser umgehen kann. Dies könnte bedeuten, dass sich Patienten schließlich weniger ohnmächtig und besser in der Lage dazu fühlen, aktiv zu handeln. Die mutmaßlich 
empfundene Enttäuschung nimmt im Therapieverlauf bei VT-Patienten mehr zu als bei SETPatienten.

Eindrücklich findet sich auch die gegenläufige Entwicklung der RS-CL 4 ,, widersetze mich und verletze andere“ (Zunahme) und CL 5 „,habe Selbstkontrolle und Selbstvertrauen“ (Abnahme) von SET-Patienten. Während die Zunahme des CL 4 als logische Schlussfolgerung aus der Auseinandersetzung mit anderen als ,feindlich " verstanden werden kann, interpretierten wir die gleichzeitige Abnahme des CL 5 nicht etwa als Indiz für fehlendes, aber vielmehr als Indiz für gefestigtes Selbstvertrauen, welches daher aus dem Fokus des Berichtens schwindet.

Die LU-RS-CL der SET-Patienten verändern sich kaum. Bemerkenswert erscheint dennoch eine relativ starke Zunahme der Häufigkeit des RS-CL ,, depressiv sein “. Wir sehen diese Reaktion, vielmehr die Bewusstwerdung von depressiven Anteilen, als Folge der intensiven Auseinandersetzung, zum Beispiel mit den Unzulänglichkeiten der anderen. Wiegand (2010) konnte in ihren Untersuchungen zeigen, dass depressive Beschwerden nicht gegen einen erfolgreichen Therapieverlauf von SET-Patienten sprechen.

Die LU-RS-CL der VT-Patienten zeigen eine deutliche Zunahme des CL ,, verärgert “ bei gleichzeitig abnehmender Häufigkeit für den CL ,unzufrieden sein, Angst haben “, woraus wir vorsichtig die Interpretation zur logisch gegenläufigen Bewegung und Veränderung ableiten, welche insbesondere vor dem Hintergrund des zunehmend bewusst werdenden „Ärgers“ als Möglichkeit zur Abgrenzung und somit als Therapieerfolg verstanden werden kann.

Fazit:

Zusammenfassend zeigen die klassischen ZBKT-CL, dass beiden Patientengruppen dieselben RO-Komponenten gemein sind und diese sich im Therapieverlauf nicht verändern. Es gibt jedoch Hinweise darauf, dass sich beide Patientengruppen gleichsam damit auseinandersetzen, andere als „feindlich" zu erleben, was wir als Therapieerfolg werten. Auch die LU-RO-CL zeigen ein ähnliches Verteilungsmuster für beide Patientengruppen. Sie lassen allerdings erkennen, dass sich SET-Patienten mehr mit dem Erleben anderer als ,feindlich" auseinandersetzen, als VT-Patienten, was wir mit dem Fokus der VT auf akute Problembewältigung begründen. Dass die LU-CL eine so feine Differenz wahrnehmen können, werten wir als klinischen Vorteil des LU-CL-Systems.

Zudem erfasst das LU-System neben dem häufigsten CL auch andere mehr oder weniger häufige CL, weshalb eine homogenere Verteilung entsteht, welche ebenfalls für eine bessere klinische Anwendbarkeit spricht.

Beide Patientengruppen weisen den häufigsten klassischen RS-CL 7 (,, bin enttäuscht und deprimiert") auf. Während er bei SET-Patienten im Therapieverlauf stabil bleibt, nimmt er bei VT-Patienten relativ gesehen deutlich zu und muss vor dem Hintergrund der gleichzeitig abnehmenden CL für „Angst“ und „Hilflosigkeit“ verstanden werden. Die Ergebnisse dürfen als Indiz für eine positive Entwicklung bei VT-Patienten hinsichtlich Problembewältigungsstrategien verstanden werden.

Während alle LU-RS-CL der SET-Patienten weitestgehend stabil bleiben, zeigen die VTPatienten Veränderungen auf, welche auf in der Therapie erlernte Fähigkeiten zur Abgrenzung schließen lassen und somit als Therapieerfolg verstanden werden dürfen.

Die Ergebnisse des AV geben weiterhin Hinweise darauf, dass Qualität und Veränderung des ZBKT`s abhängig von Therapie- und Auswertungsverfahren sind.

Viele unserer Beobachtungen zur Behandlung von GAS-Patienten mit VT und SET entsprechen denen Wiegands (2010). 


\subsection{Diskussion der Frage 5}

Gibt es Unterschiede zwischen den maßgeschneiderten ZBKT`s von VT- und SET- Patienten?

Nachdem sich die bisherigen Erläuterungen auf die Ergebnisse des AV`s stützen, folgt nun die Darstellung der maßgeschneiderten (TM-) ZBKT`s. Erneut soll ein Vergleich zwischen VT- und SET-Patienten vorgenommen werden, um der Frage nachzugehen, ob und inwiefern die Therapieform neben der bereits dargestellten Prägung des AV-ZBKTs möglicherweise auch die maßgeschneiderten Themen beeinflusst.

Für jeweils 10 VT- und 10 SET-Patienten wurden maßgeschneiderte ZBKT`s erstellt. Das Vorgehen unterschied sich insofern vom Vorgehen im AV, als es sich bei den TM-ZBKT's um individuelle Formulierungen für einzelne Patienten handelt, während sich das AV-ZBKT auf die gesamte Patientengruppe $(\mathrm{N}=45)$ bezieht. In zukünftigen Studien könnten die Untersuchungsbedingungen angeglichen und die Ergebnisse auf Signifikanz getestet werden, da wir eine rein deskriptive Darstellung vornehmen.

Das AV-ZBKT zeigt, dass sich das Wunschverhalten beider Patientengruppen im Therapieverlauf diskret verändert, sich die RO-Komponente beider Patientengruppen so gut wie nicht verändert und sich die RS-Komponente der VT-Patienten stärker wandelt als die der SET-Patienten. Das AV-ZBKT ist dennoch weitestgehend stabil und auch am Ende der Therapie noch gut $\mathrm{zu}$ erkennen, was die bereits genannten Beobachtungen Luborskys (Luborsky et al.1992, Luborsky 1999) unterstützt.

Die maßgeschneiderten Formulierungen skizzieren eine Veränderung der SET-Wünsche von "Ich will es richtig machen, sodass andere mich respektieren“ hin $\mathrm{zu}$ „Ich möchte stark sein, andere sollen mir nahe sein und tun, was ich sage". Die sich im Verlauf entwickelnde deutlich spürbare Dominanz scheint deckungsgleich mit den Beobachtungen des LU-Systems im AV-ZBKT. SET-Patienten erleben Dominanz im Sinne eines Wunsches am Therapieende bewusstseinsnäher. Auch mit den klassischen ZBKT-CL kann diese Beobachtung gemacht werden (Zunahme der Nennungshäufigkeit für CL 2 ,mich widersetzen, verletzen, kontrollieren "). Um zentrale Wunschkomponenten von SET-Patienten zu erfassen, scheinen sich das AV- und TM-Verfahren gleichermaßen zu eignen.

Eine Veränderung des Wunschverhaltens von VT-Patienten kann mit dem TM-ZBKT kaum erfasst werden. „Ich will gut sein, damit andere mich respektieren und beschützen " wird zu "Ich will es gut machen und stark sein, dass andere mich mögen “. Klassische und LU-CL konnten hingegen eine Veränderung der VT-Wünsche greifbar machen und ließen die Schlussfolgerung zu, die Patienten hätten bessere Bewältigungsstrategien erlernt und seien zu Auseinandersetzungen bereit.

Da die Beobachtungen zum ZBKT aus psychoanalytischen Sitzungen stammen, sollte in zukünftigen Studien vermehrt untersucht werden, ob die TM-Formulierung an Material aus VT-Sitzungen Anwendung finden kann.

Die TM-RO-Komponenten von VT- und SET- Patienten sind zu beiden Untersuchungszeitpunkten identisch (T1 SET: Andere sind unzuverlässig und gleichgültig, tun nicht so, wie ich will / T2 SET: Andere sind distanziert und machen nicht mit - T1 VT: Andere sind unzuverlässig, uninteressiert und gegen mich / T2 VT: Andere sind unzuverlässig und handeln gegen mich). TM- und AV-ZBKT sind deckungsgleich.

Ähnlich verhält es sich auch für die TM-RS-Komponenten von VT- und SET-Patienten (T1 SET: Ich bin enttäuscht, verunsichert, ärgerlich und bekomme Angst / T2 SET: Ich bin 
ärgerlich, enttäuscht und bekomme Angst - T1 VT: Ich bin ärgerlich, enttäuscht, unsicher und bekomme Angst / T2 VT: ich in deprimiert und wütend).

Während das AV-ZBKT von SET-Patienten weitestgehend stabil bleibt, gibt es Hinweise darauf, dass VT-Patienten Veränderungen in der Therapie durchlaufen, welche für einen Therapieerfolg sprechen. Dass sich LU-AV-ZBKT und TM-Formulierung bei SET-Patienten entsprechen, werten wir als klinischen Hinweis für die Stärke und klinische Nähe der LU-CL und schließen uns erneut Luborsky (1999) an, welcher zeigte, dass individuelle ZBKT`s auch bei gebesserten Patienten am Behandlungsende noch zu erkennen sind. Dies legt den Schluss nahe, dass maßgeschneiderte Formulierungen vielleicht sogar zu Recht ohne Veränderung bleiben, da sie der klinischen Aussage näher sind als das AV-ZBKT.

Eine weitere klinische Beobachtung (Pfeffer 1963/2004) ist die, dass Inhalte von Übertragungsmustern bestehen bleiben, jedoch weniger starr werden. Durch die in der Therapie eingetretenen - wenn auch geringen - Veränderungen imponieren Übertragungsmuster weniger dominant, sind aber grundsätzlich stabil. Diese Beobachtung kann auch mit dieser Dissertation untermauert werden. Da neue Muster parallel zu den alten, vielleicht rückläufigen Mustern auftauchen, wird das klinische Bild insgesamt „bunter“. Dies kann als Voraussetzung für eine nachhaltige Besserung der Symptomatik gewertet werden. Da wir eine rein deskriptive Darstellung vornahmen, sollten die Ergebnisse in künftigen Studien auf Reproduzierbarkeit und Signifikanz getestet werden.

Bezüglich der Besserung von Symptomen und entsprechender Korrelation zur Veränderung des ZBKT kann auf die Bestimmung der Pervasiveness zurückgegriffen werden. Luborsky und Crits-Christoph (1998, S. 160ff) sehen den Rückgang der Pervasiveness (dt. Durchziehung) theoretisch als entscheidenden kurativen Faktor, worauf hier nicht weiter eingegangen werden kann.

Hinsichtlich des unsererseits vielfach bemühten Vergleiches von VT- und SET-Patienten sollten größere Studien mit VT-Patienten aufgebaut werden, um die Reproduzierbarkeit der Ergebnisse für die VT-Patientengruppe belegen und die Anwendbarkeit der ZBKT-Methode für die VT weiter erforschen zu können. Insbesondere werden auch Verlaufsuntersuchungen nach Abschluss der Therapie notwendig sein. 


\section{Zusammenfassung}

Nach Sigmund Freud kommt Heilung durch Analyse von Übertragung zustande (vgl. Luborsky und Crits-Christoph 1998, IX). Lester Luborsky (1977, 1998a) etablierte ein Verfahren zur empirischen Messung von Übertragung (Luborsky et al. 1985) und bezeichnete es als die Methode des Zentralen Beziehungskonfliktthemas (ZBKT). Mit der ZBKT-Methode wird nach übergeordneten, zentralen Themen von Psychotherapiepatienten gesucht, die uns zu Übertragungsmustern für Verhaltensweisen in zwischenmenschlichen Beziehungen führen. Die Wiederholung des Musters lässt den darin verborgenen psychopathologischen Vorgang erkennen, wodurch es für die Therapie nutzbar wird. Die ZBKT-Methode wird von Psychotherapeuten an einer breitgefächerten Patientengruppe eingesetzt.

Für die vorliegende Dissertation wurden ZBKT`s von insgesamt 45 Patienten mit der Hauptdiagnose GAS nach dem Manual zur ZBKT-Methode von Lester Luborsky et al. (1991) bestimmt. Während 20 Patienten eine psychoanalytisch orientierte Fokaltherapie (Leichsenring 2005) erhielten, wurden 25 Patienten verhaltenstherapeutisch behandelt. Beide Therapieverfahren waren als ambulante Kurzzeittherapie konzipiert. Die Therapiesitzungen wurden im Rahmen einer teilweise von der Deutschen Forschungsgemeinschaft (DFG) geförderten randomisierten, kontrollierten klinischen Studie in der Abteilung für Psychosomatik und Psychotherapie der Universität Göttingen von Mai 2001 bis Juni 2007 aufgezeichnet.

Zur Bestimmung der Inter-Rater-Reliabilität (IRR) für die ZBKT-Methode wurden insgesamt 3 Kategoriensysteme angewendet. Es wurden sehr gute IRR erzielt, obwohl die Untersucher zuvor über keinerlei Rating-Erfahrung verfügten. Die relativ kurze Vorbereitungszeit und Schulung über zwei Hochschulsemester darf als ausreichend eingestuft werden. Aus methodischen Gründen gibt es keine Hinweise darauf, eines der Kategoriensysteme bevorzugt anzuwenden. Die IRR ist unabhängig vom Auswertungsverfahren in etwa gleich hoch. Relativ gesehen zeigen die LU-CL jedoch die besseren Übereinstimmungswerte, da sie trotz höherer Clusterzahl tendenziell bessere Ergebnisse erbringen. Mit den LU-CL (Albani et al. 2002 a,b) lassen sich insgesamt differenziertere Muster aufzeigen, weshalb sie für den klinischen Alltag bevorzugt werden sollten.

Das AV-ZBKT wurde sowohl für das Gesamtkollektiv aller 45 Patienten, als auch für die VTund SET-Untergruppe erstellt. Zudem wurden ZBKT`s des Therapieanfanges und Therapieendes ausgezählt und einander gegenüber gestellt, um Veränderungen im Therapieverlauf aufzeigen zu können. Die AV-ZBKT`s der klassischen ZBKT-CL (Barber et al. 1990) und der LU-CL (Albani et al. 2002 a,b) wurden miteinander verglichen. Für jeweils 10 VT- und SET-Patienten wurden abschließend maßgeschneiderte ZBKT`s formuliert.

Mit der vorliegenden Dissertation wurde auf einige Forderungen Albani`s (2008) hinsichtlich aktueller und zukünftiger ZBKT-Forschung eingegangen. Unter anderem wurde untersucht, ob angstgestörte Patienten ein spezifisches zentrales Thema aufweisen und ob es sich von dem anderer Patientengruppen unterscheidet. Basierend auf den Ergebnissen der vorliegenden Untersuchungen gibt es aktuell keine Hinweise dafür, dass sich mit Hilfe der ZBKT-Methode ein spezifisches ZBKT angstgestörter Patienten finden lässt. Wir schließen uns somit den Beobachtungen Crits Christoph et al. (1995) an. Individuelle Lebensverläufe prägen das ZBKT zu stark, als dass es für eine ganze Patientengruppe vereinheitlicht werden könnte. Insbesondere W- und RO der GAS-Patienten ähneln denen anderer Patientengruppen so deutlich, dass nicht zwingend von einer GAS-typischen Komponente gesprochen werden kann. Mit den LU-CL gelingt es hingegen, unterschiedliche Reaktionen (RS) verschiedener Patientengruppen differenziert aufzeigen, was für eine gute klinische Validität der ZBKT- 
Methode in dieser Kategorie spricht. Ob allein das Kriterium der Verschiedenheit und guten klinischen Validität ausreicht, um die RS-Komponente als GAS-spezifisch zu werten, bedarf weiterer Untersuchungen. Insbesondere sollten die Ergebnisse vor dem Hintergrund unserer rein deskriptiven Statistik eingeordnet werden. Weitere, ähnlich aufgebaute Untersuchungen sollten folgen, um der Frage nachzugehen, ob - und wenn ja, inwiefern - die ZBKT-Methode im Bereich der W- und RO-Komponente einen methodischen Fehler aufweist und welche Rolle unspezifische Umgebungsfaktoren wie beispielsweise das psychotherapeutische Setting spielen.

Obwohl sich kein GAS-spezifisches ZBKT finden lässt, wird dennoch die Suche nach steuernden Objekten (König 1986/2000), eine gewisse Tendenz zu vermeidendem Verhalten und unkontrollierbare Sorgen bei vielen GAS-Patienten des Patientenkollektives auf CLEbene beobachtet.

Im Rahmen der Untersuchungen der ZBKT`s von SET- und VT-Patienten stellten wir fest, dass VT- und SET-Patienten unterschiedliche, therapietypische zentrale Wunschthemen (Beziehungswunsch vs. Situationsbewältigung) aufweisen, wenn das ZBKT mit den klassischen ZBKT-CL bestimmt wird. Keine Unterschiede zeigten sich hingegen, wenn das ZBKT mit den LU-CL ermittelt wird. Als Erklärung ziehen wir entweder ein Artefakt des LU-Systems oder der klassischen CL im Wunschbereich in Betracht, tendieren aber dazu, die Verschiedenheit der Wunschthemen von SET- und VT-Patienten anzuerkennen. Wir begründen dies mit der Theorie vom steuernden Objekt (König 1986/2000) und gehen davon aus, dass die Behandlungsmethode wesentlichen Einfluss auf das Erzählen des Patienten hat. Was Therapeuten und Patienten für wichtig halten, scheint sich zu ähneln. In künftigen Studien könnte untersucht werden, wie früh sich die Ähnlichkeit der Aussagen erkennen und auch für andere Patientengruppen reproduzieren lässt. Interessanterweise sind die Unterschiede zwischen VT- und SET-Patienten bereits zum Zeitpunkt des ersten EtappenZBKT`s der dritten und fünften Therapiestunde zu erkennen. Aufgrund der eindrucksvollen Unterschiede zwischen den klassischen und LU-CL ist davon auszugehen, dass das ZBKT durch das Auswertungsverfahren wesentlich geprägt wird.

Die häufigsten Reaktionskomponenten von VT- und SET-Patienten unterscheiden sich im Gegensatz zur Wunschkomponente nicht. Allerdings zeigen die LU-CL eine breitere Verteilung über mehrere CL, wodurch sich ein wesentlich homogeneres Bild präsentiert. Wir werten dies erneut als Hinweis für eine bessere klinische Validität und strukturelle Stärke der LU-CL. Die Ergebnisse der klassischen CL weisen darauf hin, dass ein systematischer Fehler im Erfassen häufigster Kategorien vorliegen könnte, da Patienten ungeachtet ihrer Grunderkrankung konsequent auf den häufigsten RO-CL 5 und den häufigsten RS-CL 7 eingeordnet werden.

Die Untersuchung der Verlaufs-ZBKT`s zeigt sowohl unter Verwendung der klassischen ZBKT-CL als auch der LU-CL, dass sich die W-Komponenten von SET- und VT-Patienten im Verlauf diskret verändern. Während das grobe Muster bestehen bleibt, wird es weniger deutlich und wirkt weniger starr. Mit dieser Beobachtung schließen wir uns Luborsky et al. (1992) und Pfeffer (1963/2004) an. Wir interpretieren die Veränderungen als Therapieerfolg, da den Patienten - auch bei erhaltenem ZBKT - nach einer erfolgreichen Therapie bessere Bewältigungsmöglichkeiten in der konflikthaften Auseinandersetzung zur Verfügung stehen Luborsky (1999, S. 114). In einigen wesentlichen Punkten schließen wir uns den Beobachtungen Wiegands (2010) zur VT und SET bei GAS-Patienten an.

Die RO-Komponenten beider Patientengruppen bleiben im Therapieverlauf stabil, allerdings kann mit den LU-CL dokumentiert werden, dass sich SET-Patienten im Verlauf deutlicher mit dem Erleben Anderer als feindlich auseinandersetzen. Dies begründen wir mit dem Fokus der VT auf akute Problembewältigung mehr denn auf Beziehungsarbeit. Die LU-CL weisen auch 
für die RO-Komponente eine wesentlich homogenere Verteilung über mehrere CL auf, weshalb sie für den klinischen Gebrauch bevorzugt werden sollten. Die RS-Komponente der SET-Patienten bleibt stabil, während die Veränderungen bei VT-Patienten als Indiz für eine positive Entwicklung hinsichtlich Problembewältigungsstrategien verstanden werden dürfen.

Die Ergebnisse des AV geben Hinweise darauf, dass Qualität und Veränderung des ZBKT`s abhängig von Therapie- und Auswertungsverfahren sind. Während sich zeigt, dass VT- und SET-Patienten bereits zum Zeitpunkt T1 ein unterschiedliches Wunschverhalten aufweisen, so unterscheiden sich die Themen hinsichtlich ihrer Entwicklung im Therapieverlauf nicht.

Dass ZBKT-Muster im Verlauf einer Therapie bestehen bleiben, am Therapieende durch diskrete Veränderungen jedoch weniger starr wirken, wird als Therapieerfolg gewertet, da den Patienten - auch bei erhaltenem ZBKT - nach einer erfolgreichen Therapie bessere Bewältigungsmöglichkeiten zur Verfügung stehen (Luborsky 1999 S. 114; Wiegand 2010). Dass sich die Beobachtungen in beiden Behandlungsgruppen nachweisen lassen, könnte als Indiz für eine gute Anwendbarkeit psychoanalytischer Therapieverfahren bei GAS-Patienten gewertet werden, welche bislang überwiegend VT erhalten. Hinsichtlich des unsererseits vielfach bemühten Vergleiches des ZBKT`s von VT- und SET-Patienten sollten größere Studien mit VT-Patienten aufgebaut werden, um die Anwendbarkeit der ZBKT-Methode für die VT zu prüfen. Aktuell müssen die Ergebnisse vor dem Hintergrund der deskriptiven Statistik eingeordnet werden.

Abschließend wurde untersucht, ob sich maßgeschneiderte ZBKT`s von VT- und SETPatienten unterscheiden. Wir stellten fest, dass sich AV und maßgeschneiderte Verfahren gleichermaßen eignen, um zentrale Wunschkomponenten von SET-Patienten zu erfassen. Mit beiden Verfahren ließen sich dieselben - im Sinne eines Therapieerfolges gewerteten Veränderungen dokumentieren. Bei VT-Patienten konnten die maßgeschneiderten Formulierungen die Veränderungen nicht erfassen. Da die Beobachtungen zum ZBKT aus psychoanalytischen Sitzungen stammen schlagen wir vor, in zukünftigen Studien erneut zu prüfen, ob maßgeschneiderte ZBKT`s an VT-Sitzungen Anwendung finden können. Wiederholt fanden wir Hinweise darauf, dass sich maßgeschneiderte ZBKT's und LUFormulierung besonders in der Wunsch- und RS-Kategorie qualitativ stark ähneln, weshalb wir von einer besonderen klinischen Nähe dieser beiden Verfahren ausgehen. 


\section{Anhang}

Standardkategorien der ZBKT-Methode (Crits-Christoph und Demorest (1988), zitiert nach Barber et al. (1990)). Übersetzung in Luborsky et al. (1991, S. 25-27).

\section{ZBKT-STANDARDKATEGORIEN FÜR WÜNSCHE/ABSICHTEN}

1. VERSTANDEN WERDEN; Verständnis bekommen; Mitgefühl bekommen; richtig gesehen werden

2. ANGENOMMEN/AKZEPTIERT WERDEN; von anderen gut beurteilt/ nicht verurteilt werden; Bestätigung erhalten

3. RESPEKTIERT WERDEN; geachtet, geschätzt werden; gerecht behandelt werden; anderen wichtig sein

4. ANDERE ANNEHMEN/AKZEPTIEREN; für andere aufgeschlossen/ empfänglich sein

5. ANDERE RESPEKTIEREN/ACHTEN; andere anerkennen

6. VERTRAUEN HABEN; Ehrlichkeit, Aufrichtigkeit bei anderen

7. BELIEBT SEIN; gemocht werden; Interesse von anderen entgegengebracht bekommen

8. OFFENHEIT VON ANDEREN; andere sollen auf mich eingehen, mit mir sprechen

9. KOMMUNIKATION MIT ANDEREN; offen sein; mich ausdrücken können

10. ABSTAND VON ANDEREN HABEN; nicht offen sein; meinen Gefühlen nicht Ausdruck geben; in Ruhe gelassen werden

11. ANDEREN NAHE SEIN; einbezogen werden; nicht alleine sein; befreundet sein

12. ANDEREN HELFEN; anderen beistehen; anderen geben

13. HILFE/BEISTAND BEKOMMEN; Unterstützung bekommen; etwas Wertvolles bekommen; beschützt werden

14. NICHT VERLETZT WERDEN; Schmerzliches vermeiden; Zurückweisung vermeiden; mich schützen/verteidigen

15. VERLETZT WERDEN; bestraft werden; schlecht behandelt werden

16. ANDERE VERLETZEN; mich rächen; andere zurückweisen; meinem Ärger Luft machen

17. AUSEINANDERSETZUNGEN VERMEIDEN; Kompromisse schließen; andere nicht verärgern; mit anderen auskommen; flexibel sein;

18. MICH ANDEREN WIDERSETZEN; gegen Beherrschung Widerstand leisten; mit anderen in Konkurrenz treten

19. ÜBER ANDERE KONTROLLE/MACHT HABEN; dominieren; meine Vorstellungen durchsetzen; Anerkennung meiner Rechte erzwingen

20. VON ANDEREN KONTROLLIERT WERDEN; Anweisungen bekommen; passiv, unterwürfig, abhängig sein

21. SELBSTKONTROLLE HABEN; rational sein; berechenbar sein

22. ERFOLG HABEN; kompetent sein; gewinnen; berühmt sein

23. UNABHÄNGIG SEIN; autonom sein

24. SELBSTVERTRAUEN HABEN; mich gut fühlen; mich akzeptieren 


\section{ZBKT-STANDARDKATEGORIEN FÜR REAKTIONEN DES OBJEKTS (RO)}

1. HABEN VERSTÄNDNIS; haben Mitgefühl; sind einfühlsam; sehen mich richtig

2. VERSTEHEN NICHT; haben kein Mitgefühl; sind nicht einfühlsam; nehmen keine Rücksicht

3. NEHMEN MICH AN/AKZEPTIEREN; weisen nicht zurück; sind mit mir einverstanden; beziehen mich ein

4. SIND ZURÜCKWEISEND; sind nicht einverstanden; sind kritisch

5. RESPEKTIEREN MICH; behandeln mich gerecht; schätzen mich; bewundern mich

6. RESPEKTIEREN MICH NICHT; behandeln mich ungerecht; schätzen mich nicht; bewundern mich nicht

7. VERTRAUEN MIR NICHT; glauben mir nicht; sind misstrauisch

8. SIND NICHT VERTRAUENSWÜRDIG; betrügen mich; sind betrügerisch; sind unehrlich

9. MÖGEN MICH; haben Interesse an mir

10. MÖGEN MICH NICHT; sind nicht an mir interessiert

11. SIND AUFRICHTIG; sind offen; sind verfügbar

12. SIND DISTANZIERT; reagieren nicht; sind nicht verfügbar

13. SIND HILFSBEREIT; unterstützen; geben mir; erklären

14. SIND NICHT HILFSBEREIT; unterstützen nicht; geben keinen Beistand

15. VERLETZEN MICH; sind gewaltsam; behandeln mich schlecht; bestrafen

16. SIND VERLETZT; sind verwundet; sind getroffen

17. WIDERSETZEN SICH MIR; konkurrieren; lehnen meine Wünsche ab; stellen sich gegen mich

18. SIND SYMPATHISCH; sind angenehm; sind kooperativ

19. SIND UNZUVERLÄSSIG; sind verantwortungslos; sind ausserhalb meiner Kontrolle

20. SIND BEHERRSCHEND; sind dominant; schüchtern ein; sind aggressiv; übernehmen das Kommando

21. GEBEN MIR UNABHÄNGIGKEIT; Autonomie; ermutigen zur Selbstbestimmung

22. SIND ABHÄNGIG; sind von mir beeinflusst; sind unterwürfig

23. SIND UNABHÄNGIG; bestimmen selbst; passen sich nicht an; sind autonom

24. SIND STARK; sind überlegen; sind verantwortlich; sind bedeutend

25. SIND SCHLECHT; sind im Unrecht; sind schuldig

26. SIND STRENG; sind stur; sind hart

27. SIND VERÄRGERT; sind wütend; sind gereizt; vorwurfsvoll; frustriert

28. SIND ÄNGSTLICH; sind besorgt; sind nervös

29. SIND GLÜCKLICH; sind ausgelassen; genießen; sind unterhaltsam

30. LIEBEN MICH; sind an mir romantisch interessiert 


\section{ZBKT-STANDARDKATEGORIEN FÜR REAKTIONEN DES SUBJEKTS (RS)}

1. HABE VERSTÄNDNIS; verstehe; erkenne; sehe etwas richtig

2. VERSTEHE NICHT; bin verwirrt; bin überrascht; verstehe mich selbst nicht

3. FÜHLE MICH ANGENOMMEN/AKZEPTIERT

4. FÜHLE MICH RESPEKTIERT/GEACHTET; fühle mich geschätzt, bewundert

5. MAG ANDERE; bin freundlich

6. MAG ANDERE NICHT; hasse andere

7. SPRECHE MICH AUS; bin offen; kommuniziere

8. SPRECHE NICHT AUS; bin nicht offen; bin gehemmt; bin distanziert

9. BIN HILFSBEREIT; unterstütze; versuche anderen zu gefallen; gebe

10. VERLETZE ANDERE; bin gewaltsam; handele feindselig

11. WIDERSETZE MICH ANDEREN; trete in Konkurrenz, in Auseinandersetzung; verweigere mich/etwas anderen

12. DOMINIERE; kontrolliere; habe Einfluss; manipuliere andere; bin bestimmend; bin aggressiv

13. BIN UNZUVERLÄSSIG; entziehe mich der Kontrolle; bin impulsiv

14. HABE SELBSTKONTROLLE; bin verantwortungsbewusst

15. BIN UNABHÄNGIG; entscheide selbst; bin autonom

16. BIN ABHÄNGIG; bin unterwürfig; bin passiv; bin unfrei

17. BIN UNFÄHIG; bin inkompetent; bin hilflos

18. FÜHLE MICH SELBSTSICHER; bin oder fühle mich erfolgreich; fühle mich stolz

19. BIN UNSICHER; bin ambivalent; fühle mich hin- und hergerissen

20. FÜHLE MICH ENTTÄUSCHT; bin unbefriedigt; fühle mich verstimmt; fühle mich unausgefüllt

21. FÜHLE MICH VERÄRGERT; fühle mich wütend; gereizt; frustriert

22. FÜHLE MICH DEPRIMIERT; fühle mich ohne Hoffnung; traurig

23. FÜHLE MICH UNGELIEBT; fühle mich einsam; zurückgewiesen

24. FÜHLE MICH EIFERSÜCHTIG; fühle mich neidisch

25. FÜHLE MICH SCHULDIG; fühle mich im Unrecht

26. SCHÄME MICH; fühle mich peinlich berührt; bin beschämt

27. FÜHLE MICH ÄNGSTLICH; fühle mich besorgt; nervös

28. FÜHLE MICH WOHL; fühle mich sicher; in Sicherheit; bin oder fühle mich zufrieden

29. FÜHLE MICH GLÜCKLICH; fühle mich froh; ausgelassen; genieße

30. FÜHLE MICH GELIEBT; fühle mich in einer romantischen Beziehung 


\section{ZBKT-STANDARDKATEGORIEN: CLUSTER FÜR W, RO UND RS}

Cluster der Standardkategorien für die ZBKT-Methode (Barber et al. 1990, Übersetzung in Luborsky et al. 1991, S. 28)

\section{WÜNSCHE Enthält W Nr.}

(Ich will:)

1. Mich behaupten und unabhängig sein 21, 23, 28, 34

2. Mich anderen widersetzen und andere verletzen und kontrollieren 16, 18, 19

3. Kontrolliert und verletzt werden und keine Verantwortung haben 13, 15, 20, 27, 29

4. Abstand haben und Auseinandersetzung vermeiden 10, 14, 17

5. (Anderen) nahe sein und annehmen 4, 5, 6, 8, 9, 11

6. Geliebt und verstanden werden 1, 2, 3, 7, 33

7. Mich gut und wohl fühlen 24, 30, 31, 32

8. (Erfolg, Leistung) erreichen und anderen helfen 12, 22, 25, 26

\section{REAKTIONEN DES OBJEKTS Enthält RO Nr.}

(Andere sind/tun:)

1. Stark 23, 24, 29

2. Kontrollieren 20, 26

3. Bestürzt, aufgeregt und ärgerlich 16, 19, 22, 27, 28

4. Schlecht 8, 25

5. Weisen zurïck und sind gegen mich $2,4,6,7,10,12,14,15,17$

6. Hilfreich 13, 18

7. Mögen mich 5, 9, 21, 30

8. Verstehen $1,3,11$

REAKTIONEN DES SUBJEKTS Enthält RS Nr.

(Fühle mich/bin/tue/habe:)

1. Hilfreich 1, 7, 9

2. Unempfänglich und unaufgeschlossen $2,6,8$

3. Respektiert und akzeptiert 3, 4, 5, 28, 29,30

4. Widersetze mich und verletze andere 10, 11

5. Selbstkontrolle und Selbstvertrauen 12, 14, 15, 18

6. Hilflos 13, 16, 17, 19

7. Enttäuscht und deprimiert 20, 21, 22, 23, 24

8. Ängstlich und beschämt 25, 26, 27 


\title{
Alternative Cluster der Standardkategorien
}

(Körner et al. 2002, S. 52-54), auf der Grundlage des bestehenden Systems von Standardkategorien (Crits-Christoph und Demorest 1988), in ihrer deutschen Übersetzung neu berechnet.

\author{
ZBKT-STANDARDKATEGORIEN: CLUSTER FÜR W, RO UND RS \\ WÜNSCHE Enthält W Nr. \\ (Ich will:) \\ D1. unabhängig und nicht verpflichtet sein 23, 28, 29 \\ D2. mich widersetzen und andere dominieren 16, 18, 19, 34 \\ D3. verletzt und kontrolliert werden 15, 20, 27 \\ D4. Konflikte vermeiden 10, 14, 17 \\ D5. andere annehmen 4, 5 \\ D6. offen kommunizieren 6, 8, 9 \\ D7. akzeptiert und unterstützt werden 1, 2, 3, 13, 30 \\ D8. geliebt werden 7, 11, 33 \\ D9. mich wohl fühlen 31,32 \\ D10. Selbstvertrauen haben und anderen helfen 12, 21, 22, 24, 25, 26
}

\section{REAKTIONEN DES OBJEKTS Enthält RO Nr.}

(Andere sind/tun:)

D1. autonom und beherrschend 15, 17, 20, 23, 24, 26

D2. verletzt und ängstlich 16, 22, 25, 27

D3. unzuverlässig 8, 19, 25

D4. weisen zurück und verstehen nicht 2, 4, 6, 7

D5. distanziert und verweigern sich 10, 12, 14

D6. sind hilfreich $11,13,18,21$

D7. mögen mich $9,29,30$

D8. akzeptieren und verstehen mich 1, 3, 5

\section{REAKTIONEN DES SUBJEKTS Enthält RS Nr.}

(Fühle mich/bin/tue/habe:)

D1. offen und hilfreich 1, 5, 7, 9

D2. entziehe mich 8,13

D3. geachtet und angenommen 3, 4

D4. wohl und geliebt 28, 29, 30

D5. widersetze mich und beherrsche $10,11,12$

D6. autonom 14, 15, 18

D7. unsicher und ängstlich 2, 16, 17, 19, 27

D8. verärgert und verstimmt $6,20,21,24$

D9. ungeliebt und deprimiert 22, 23

D10. schäme mich 25, 26 
Prädikate des reformulierten ZBKT-Systems (Albani et al. 2002 b, S. 229-230)

\begin{tabular}{|c|c|c|}
\hline \multirow{11}{*}{ 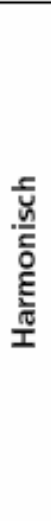 } & \multirow{2}{*}{ A sich Zuwenden } & A1 explodieren, bewundern \\
\hline & & A2 akzeptieren, verstehen \\
\hline & \multirow{2}{*}{ B unterstützen } & B1 erklären, bestätigen \\
\hline & & B2 helfen \\
\hline & \multirow{5}{*}{$C$ lieben, sich wohlfūhlen } & C1 nahe sein \\
\hline & & C2 lieben \\
\hline & & C3 zuversichtlich, zufrieden, sinnlich vergnūgt \\
\hline & & C4 sexuell aktiv sein \\
\hline & & C 5 gesund sein \\
\hline & \multirow{2}{*}{ D souverän sein } & D1 stark sein \\
\hline & & D2 stolz, autonom sein \\
\hline \multirow{19}{*}{ 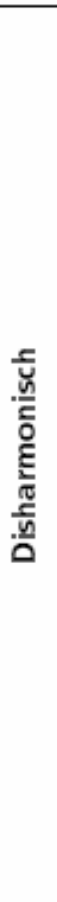 } & \multirow{2}{*}{ E depressiv sein, resignieren } & E1 enttäuscht sein \\
\hline & & E2 resignieren \\
\hline & \multirow{2}{*}{ F unzufrieden sein, Angst haben } & F1 Schuld/Scham empfinden, unzufrieden sein \\
\hline & & F2 sich fürchten, ängstlich sein \\
\hline & \multirow{2}{*}{ G fremdbestimmt sein } & G1 abhängig sein \\
\hline & & G2 schwach sein \\
\hline & \multirow{2}{*}{ H verärgert, unsympathisch sein } & H1 Ekel empfinden, verärgert sein \\
\hline & & H2 unsympathisch sein \\
\hline & \multirow{2}{*}{ I unzuverlässig sein } & I1 vernachlässigen \\
\hline & & I2 egozentrisch sein \\
\hline & \multirow{2}{*}{$\mathrm{J}$ zurūckweisen } & J1 ignorieren, vorwerfen \\
\hline & & J2 widersetzen, kritisieren \\
\hline & \multirow{2}{*}{ K dominieren } & K1 schlecht sein \\
\hline & & K2 beherrschen \\
\hline & \multirow{2}{*}{ L ärgern, angreifen } & L1 jemanden ärgern \\
\hline & & L2 angreifen \\
\hline & \multirow{3}{*}{ M sich zurūckziehen } & M1 sich zurückziehen, sich zurückhalten \\
\hline & & M2 sexuell aktiv sein \\
\hline & & M3 krank sein \\
\hline
\end{tabular}




\begin{tabular}{|c|c|c|}
\hline \multirow{13}{*}{ A sich zuwenden } & \multirow{6}{*}{$\begin{array}{l}\text { A1 explodieren, } \\
\text { bewundern }\end{array}$} & $\begin{array}{l}\text { A11 neugierig sein, interessieren, erforschen, } \\
\text { motiviert sein, offen sein }\end{array}$ \\
\hline & & $\begin{array}{l}\text { A12 zu sich selbst finden, suchen, sich für et was } \\
\text { einsetzen }\end{array}$ \\
\hline & & A13 für fähig halten \\
\hline & & A14 bewundern, beeindruckt sein \\
\hline & & A15 begeistert sein, fasziniert sein \\
\hline & & A16 sich identifizieren, wie der andere sein \\
\hline & \multirow{7}{*}{$\begin{array}{l}\text { A2 akzeptieren, } \\
\text { verstehen }\end{array}$} & A21 akzeptieren, respektieren, ernst nehmen \\
\hline & & $\begin{array}{l}\text { A22 Unabhängigkeit geben, aufmerksam sein, } \\
\text { in Ruhe lassen }\end{array}$ \\
\hline & & $\begin{array}{l}\text { A23 zugehen, beachten, eingehen, zuhören, } \\
\text { ent schuldigen }\end{array}$ \\
\hline & & $\begin{array}{l}\text { A24 Gefühle wahrnehmen, ,Gefūhle zulassen, } \\
\text { empfindlich sein, sensibel sein }\end{array}$ \\
\hline & & A25 bemitleiden, berūhrt sein, gerührt sein \\
\hline & & A26 verstehen \\
\hline & & A27 verzeihen, versōhnen \\
\hline \multirow{5}{*}{ B unterstūtzen } & \multirow{2}{*}{ B1 erklären, bestätigen } & $\begin{array}{l}\text { B11 erklären, kommunizieren, aussprechen, } \\
\text { ausdrücken, überzeugen }\end{array}$ \\
\hline & & $\begin{array}{l}\text { B12 zu jmd. Halten, loben, einverstanden, an } \\
\text { regen, ermuntern }\end{array}$ \\
\hline & \multirow{3}{*}{ B2 helfen } & B21 beschützen \\
\hline & & B22 großzūgig sein, verwōhnen, bevorzugen \\
\hline & & B23 helfen, sich für jmd. Einzusetzen \\
\hline \multirow{10}{*}{ C lieben, sich wohl fühlen } & \multirow{3}{*}{ C1 nahe sein } & $\begin{array}{l}\text { C11 nahe sein, annehmen, Nähe geben, ver sor- } \\
\text { gen, gut sein, liebevoll sein }\end{array}$ \\
\hline & & C12 trōsten, beruhigen \\
\hline & & $\begin{array}{l}\text { C13 mögen, beliebt sein, sympathisch sein, } \\
\text { Freundschaft haben, sich verstehen }\end{array}$ \\
\hline & \multirow{3}{*}{ C2 lieben } & C21 verlieben, attraktiv sein \\
\hline & & C22 lieben \\
\hline & & $\begin{array}{l}\text { C23 Kinder haben, Beziehung/Partnerschaft ha- } \\
\text { ben }\end{array}$ \\
\hline & \multirow{4}{*}{$\begin{array}{l}\text { C3 zuversichtlich sein/ } \\
\text { sinnliches Vergnü- } \\
\text { gen erleben }\end{array}$} & $\begin{array}{l}\text { C31 vertrauen, sicher sein, glauben, zuver sicht- } \\
\text { lich sein, geborgen sein }\end{array}$ \\
\hline & & C32 erleichtert sein \\
\hline & & $\begin{array}{l}\text { C33 sich gehen lassen, spontan sein, sich frei } \\
\text { entfalten, glücklich sein, sich wohl fühlen, } \\
\text { genießen, Spaß haben }\end{array}$ \\
\hline & & $\begin{array}{l}\text { C34 sich freuen, (freudig) überrascht sein, zu } \\
\text { frieden sein }\end{array}$ \\
\hline
\end{tabular}




\begin{tabular}{|c|c|c|}
\hline \multirow{7}{*}{ C lieben, sich wohl fühlen } & \multirow{5}{*}{ C4 sexuell aktiv sein } & C41 romantisch sein \\
\hline & & C42 Annäherungsversuche machen, flirten \\
\hline & & C43 berühren, küssen, streicheln, zärtlich sein \\
\hline & & $\begin{array}{l}\text { C44 begehren, erregt sein, Lust haben, sexuell } \\
\text { attraktiv sein }\end{array}$ \\
\hline & & $\begin{array}{l}\text { C45 Sex haben, triebhaft sein, potent sein, lei } \\
\text { denschaftlich sein, sexuell erfahren sein }\end{array}$ \\
\hline & \multirow{2}{*}{ C5 gesund sein } & C51 gesund sein \\
\hline & & C52 leben \\
\hline \multirow{16}{*}{ D souverän sein } & \multirow{8}{*}{ D1 stark sein } & D11 dankbar sein \\
\hline & & D12 tolerant, kompromissbereit sein \\
\hline & & $\begin{array}{l}\text { D13 rücksichtsvoll. Höflich sein, maßvoll sein, } \\
\text { bescheiden sein }\end{array}$ \\
\hline & & D14 gelassen sein, geduldig sein \\
\hline & & $\begin{array}{l}\text { D15 aushalten, erdulden, durchstehen, bewäl } \\
\text { tigen }\end{array}$ \\
\hline & & $\begin{array}{l}\text { D16 vertrauenswūrdig, ehrlich sein, zuverlässig } \\
\text { sein, treu sein, gerecht behandeln, korrekt } \\
\text { sein }\end{array}$ \\
\hline & & D17 vernünftig, konstruktiv sein \\
\hline & & D18 Verantwortung tragen \\
\hline & \multirow{8}{*}{ D2 stolz, autonom sein } & $\begin{array}{l}\text { D21 stark sein, überlegen sein, wichtig sein, } \\
\text { mutig sein, s ich entscheiden }\end{array}$ \\
\hline & & $\begin{array}{l}\text { D22 fähig sein, erfahren sein, erfolgreich sein, } \\
\text { stolz sein }\end{array}$ \\
\hline & & D23 ehrgeizig sein, fleißig sein \\
\hline & & D24 Vorbild sein, perfekt sein \\
\hline & & D25 unabhängig sein, selbstständig sein \\
\hline & & $\begin{array}{l}\text { D26 selbstsicher sein, Selbstvertrauen haben, } \\
\text { selbstbewusst sein }\end{array}$ \\
\hline & & $\begin{array}{l}\text { D27 Selbstkontrolle haben, nachdenklich sein, } \\
\text { skeptisch sein, selbstkritisch sein }\end{array}$ \\
\hline & & D28 verändern, sich entwickeln, bessern \\
\hline \multirow{4}{*}{$\begin{array}{l}\text { E depressiv sein/ } \\
\text { resignieren }\end{array}$} & \multirow[t]{2}{*}{ E1 enttäuscht sein } & $\begin{array}{l}\text { E11 unglücklich sein, deprimiert sein, ent- } \\
\text { täuscht sein }\end{array}$ \\
\hline & & E12 verzweifelt sein, leiden, trauern \\
\hline & \multirow[b]{2}{*}{ E2 resignieren } & E21 aufgeben, resignieren \\
\hline & & $\begin{array}{l}\text { E22 gleichgültig sein, langweilen, lustlos sein, } \\
\text { träge sein }\end{array}$ \\
\hline
\end{tabular}




\begin{tabular}{|c|c|c|c|}
\hline \multirow{8}{*}{\multicolumn{2}{|c|}{$\begin{array}{l}\text { unzufrieden sein, } \\
\text { Angst haben }\end{array}$}} & \multirow{4}{*}{\begin{tabular}{|ll} 
F1 & Schuld/Scham \\
& empfinden/ unzu- \\
& frieden sein
\end{tabular}} & F11 schuldig fühlen, bereuen \\
\hline \multirow[b]{4}{*}{$\mathrm{F}$} & & & F12 schämen \\
\hline & & & F13 sich unwohl fühlen, unzufrieden sein \\
\hline & & & F14 sich frustriert fūhlen \\
\hline & & \multirow{4}{*}{$\begin{array}{l}\text { F2 sich fürchten ängst- } \\
\text { lich sein }\end{array}$} & $\begin{array}{l}\text { F21 ängstlich sein, sich fürchten, besorgt sein, } \\
\text { ausweichen, feige sein }\end{array}$ \\
\hline & & & $\begin{array}{l}\text { F22 unsicher sein, verwirrt sein, unentschlossen } \\
\text { sein }\end{array}$ \\
\hline & & & $\begin{array}{l}\text { F23 nervōs sein, hysterisch sein, angespannt ein, } \\
\text { unbeherrscht sein }\end{array}$ \\
\hline & & & $\begin{array}{l}\text { F24 erschrecken, entsetzt sein, sich ertappt füh- } \\
\text { len }\end{array}$ \\
\hline & \multirow{9}{*}{ fremdbestimmt sein } & \multirow{4}{*}{ G1 abhängig sein } & G11 allein sein, jemanden vermissen, einsam sein \\
\hline & & & G12 abhängig sein klammern \\
\hline & & & G13 unselbstständig sein, selbst unsicher sein \\
\hline & & & $\begin{array}{l}\text { G14 passiv sein, zweifeln, verharren, stillstehen, } \\
\text { verschlechtern }\end{array}$ \\
\hline & & \multirow{5}{*}{ G2 schwach sein } & $\begin{array}{l}\text { G21 schwach sein, hilflos sein, rechtlos sein, aus- } \\
\text { geliefert sein, ungeschützt sein, unterlegen } \\
\text { sein, verletzt sein }\end{array}$ \\
\hline & & & G22 unfähig sein, unerfahren sein \\
\hline & & & $\begin{array}{l}\text { G23 jemanden enttäuschen, überfordert sein, } \\
\text { versagen }\end{array}$ \\
\hline & & & $\begin{array}{l}\text { G24 minderwertig sein, unwichtig sein, hässlich } \\
\text { sein }\end{array}$ \\
\hline & & & G25 maßvoll sein (aus Schwäche) \\
\hline & \multirow{10}{*}{$\begin{array}{l}\text { verärgert/ } \\
\text { unsympathisch sein }\end{array}$} & \multirow{7}{*}{$\begin{array}{l}\text { H1 Ekel empfinden/ } \\
\text { verärgert sein }\end{array}$} & H11 Ekel empfinden \\
\hline & & & H12 verachten \\
\hline & & & H13 eifersüchtig sein, neidisch sein \\
\hline & & & H14 gekränkt sein, beleidigt sein \\
\hline & & & H15 nicht mögen \\
\hline & & & $\begin{array}{l}\text { H16 verärgert sein, wūtend sein, frustriert sein } \\
\text { durch etwas }\end{array}$ \\
\hline & & & H17 hassen \\
\hline & & \multirow{3}{*}{ H2 unsympathisch sein } & H21 nachtragend sein, ungeduldig sein \\
\hline & & & H22 geizig sein \\
\hline & & & $\begin{array}{l}\text { H23 unsympathisch sein, unbeliebt sein, uninter- } \\
\text { essant sein }\end{array}$ \\
\hline \multirow{5}{*}{\multicolumn{2}{|c|}{ I unzuverlässig sein }} & \multirow{2}{*}{ I1 vernachlässigen } & $\begin{array}{l}\text { I11 } \begin{array}{l}\text { unsensibel sein, kein Verständnis haben, de- } \\
\text { struktiv sein, unvernünftig, unkontrolliert } \\
\text { sein }\end{array} \\
\end{array}$ \\
\hline & & & $\begin{array}{l}\text { I12 vernachlässigen, im Stich lassen, oberfläch- } \\
\text { lich, verantwortungslos, gefühllos sein, faul } \\
\text { sein }\end{array}$ \\
\hline & & \multirow{3}{*}{ I2 egozentrisch sein } & I21 selbstgefällig sein, unkritisch sein \\
\hline & & & I22 unehrlich sein, ungerecht sein \\
\hline & & & I23 egoistisch sein, gierig sein \\
\hline
\end{tabular}




\begin{tabular}{|c|c|c|}
\hline \multirow{4}{*}{ J zurückweisen } & \multirow{2}{*}{$\begin{array}{l}\text { J1 ignorieren, vorwer- } \\
\text { fen }\end{array}$} & $\begin{array}{l}\text { J11 verunsichern, den Mut nehmen, entwerten, } \\
\text { desinteressiert sein, ignorieren }\end{array}$ \\
\hline & & $\mathrm{J} 12$ beschuldigen, vorwerfen \\
\hline & \multirow{2}{*}{$\begin{array}{l}\text { J2 widersetzen, kriti- } \\
\text { sieren }\end{array}$} & $\begin{array}{l}\text { J21 widersetzen, konkurrieren, stur sein, strei- } \\
\text { ten }\end{array}$ \\
\hline & & $\begin{array}{l}\text { J22 zurückweisen, ausschließen, kritisieren, er- } \\
\text { mahnen, ablehnen, verurteilen, tadeln }\end{array}$ \\
\hline \multirow{4}{*}{$\mathrm{K}$ dominieren } & \multirow[t]{2}{*}{ K1 schlecht sein } & $\begin{array}{l}\text { K11 schlecht sein, aus-/benutzen, betrügen, verra } \\
\text { ten, verleugnen, stehlen }\end{array}$ \\
\hline & & K12 einschmeicheln, intrigieren, täuschen \\
\hline & \multirow[b]{2}{*}{ K2 beherrschen } & $\begin{array}{l}\text { K21 verpflichten, vorschreiben, beeinflussen, un- } \\
\text { ter Druck setzen, fordern, zu etwas zwingen }\end{array}$ \\
\hline & & $\begin{array}{l}\text { K22 beherrschen, durchsetzen, verdrängen, her- } \\
\text { ab setzen, unterwerfen, benachteiligen, } \\
\text { kontrol lieren, jemanden prüfen, streng sein }\end{array}$ \\
\hline \multirow{8}{*}{$\mathrm{L}$ ärgern, angreifen } & \multirow{4}{*}{ L1 jemanden ärgern } & $\begin{array}{l}\text { L11 kränken, beleidigen, blamieren, lächerlich } \\
\text { machen, demütigen }\end{array}$ \\
\hline & & L12 schadenfroh, zynisch sein, auslachen \\
\hline & & $\begin{array}{l}\text { L13 ärgern, belästigen, hemmen, jemanden be } \\
\text { lasten }\end{array}$ \\
\hline & & L14 stōren, ablenken \\
\hline & \multirow{4}{*}{ L2 angreifen } & $\begin{array}{l}\text { L21 Angst machen, bedrohen, angreifen, provo- } \\
\text { zieren }\end{array}$ \\
\hline & & $\begin{array}{l}\text { L22 quälen, kōrperlich verletzen, feindselig sein, } \\
\text { kaputtmachen }\end{array}$ \\
\hline & & $\begin{array}{l}\text { L23 bestrafen, rächen, zerstören, Gewalt anwen- } \\
\text { den }\end{array}$ \\
\hline & & L24 issbrauchen, vergewaltigen \\
\hline \multirow{15}{*}{ M sich zurückziehen } & \multirow{8}{*}{$\begin{array}{l}\text { M1 sich zurück ziehen/ } \\
\text { zurückhalten }\end{array}$} & M11 verlassen, distanzieren, abgrenzen \\
\hline & & M12 Abstand halten, zurückziehen, entziehen \\
\hline & & M13 misstrauisch sein \\
\hline & & $\begin{array}{l}\text { M14 Auseinandersetzung vermeiden, angepasst } \\
\text { sein, recht machen, nachgeben, sich unter- } \\
\text { werfen }\end{array}$ \\
\hline & & M15 verschlossen sein, schweigen \\
\hline & & M16 schūchtern, zurückhaltend sein \\
\hline & & M17 zwanghaft sein \\
\hline & & $\begin{array}{l}\text { M18 keine Kinder haben, keine Beziehung/Part- } \\
\text { nerschaft haben }\end{array}$ \\
\hline & \multirow{3}{*}{ M2 sexuell inaktiv sein } & M21 abgeneigt sein, es über sich ergehen lassen \\
\hline & & $\begin{array}{l}\text { M22 verklemmt sein, nicht erregt sein, impotent } \\
\text { sein }\end{array}$ \\
\hline & & M23 sexuell unerfahren sein \\
\hline & \multirow{4}{*}{ M3 krank sein } & M31 erschöpft, müde sein \\
\hline & & M32 Symptome haben \\
\hline & & M33 körperlich krank sein, seelisch krank sein \\
\hline & & M34 sterben sich umbringen \\
\hline
\end{tabular}


Auswertungsbogen 1

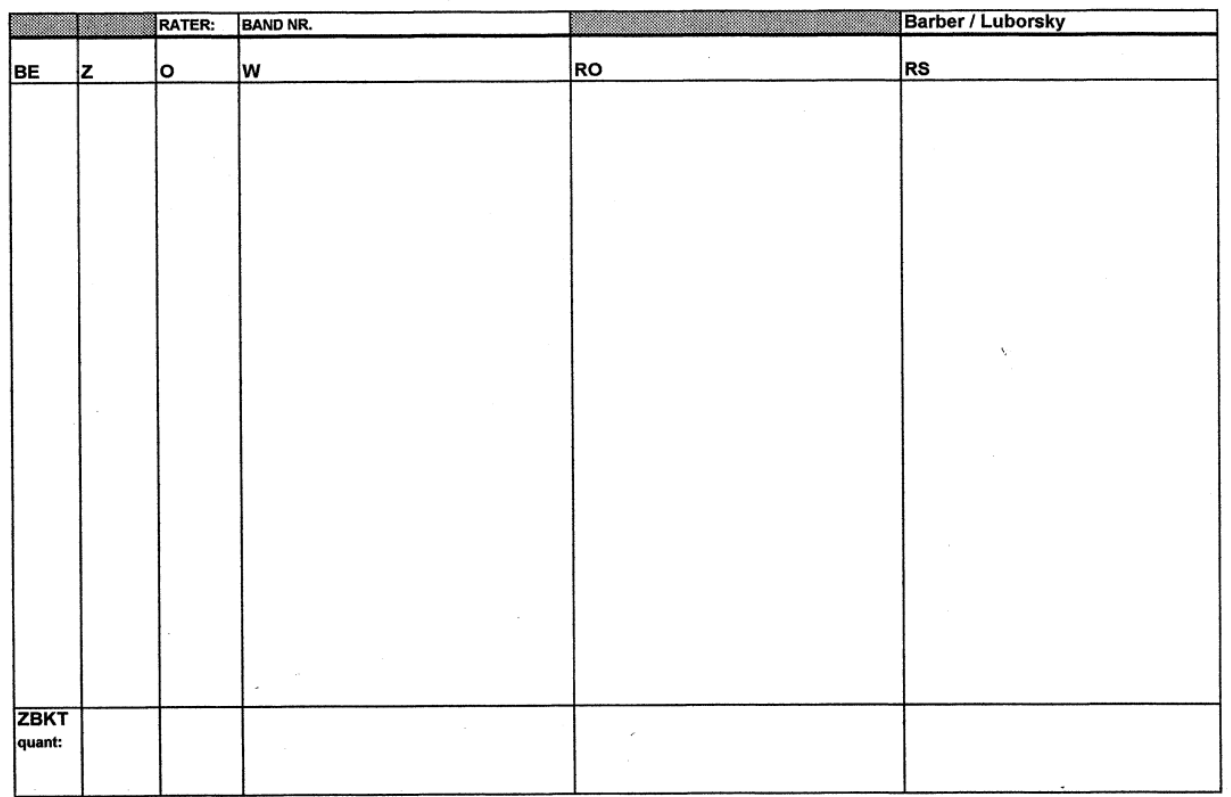


Auswertungsbogen 2

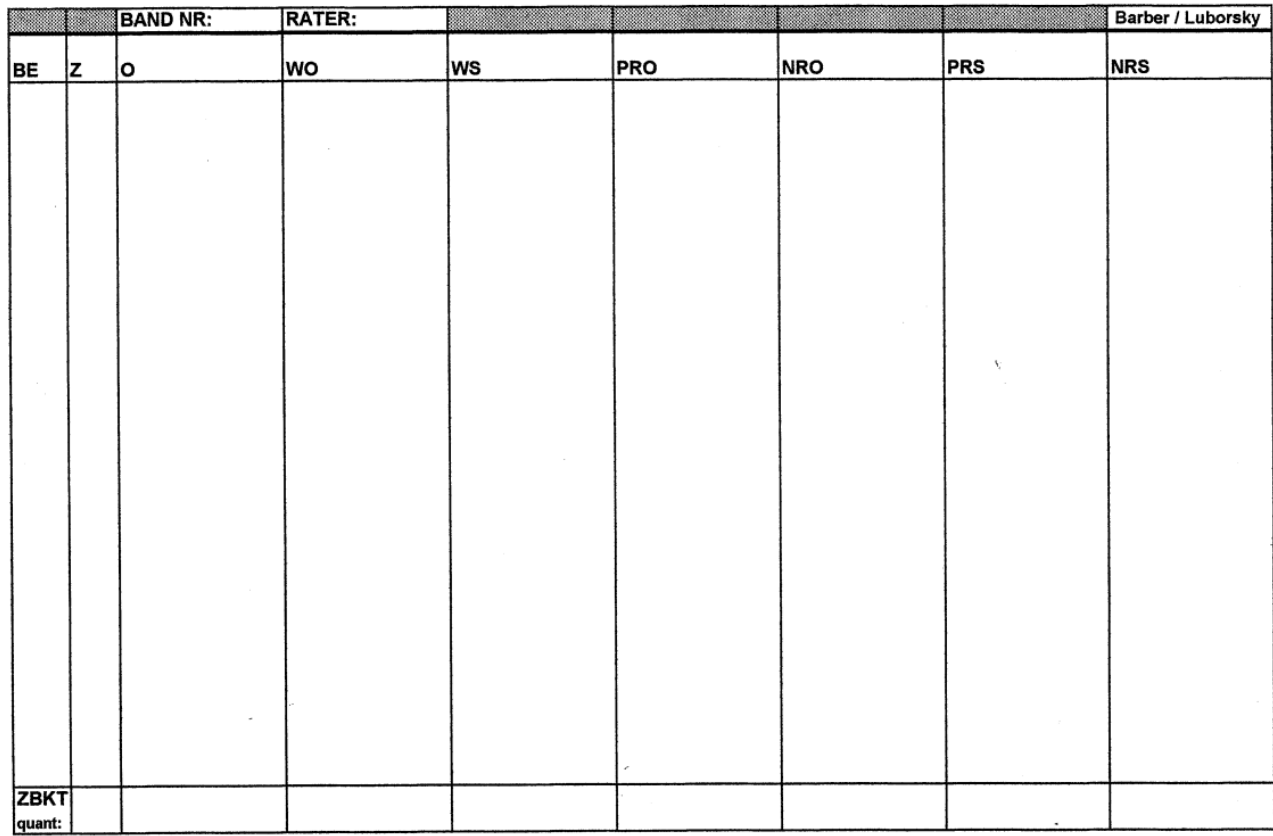


Auswertungsbogen 3

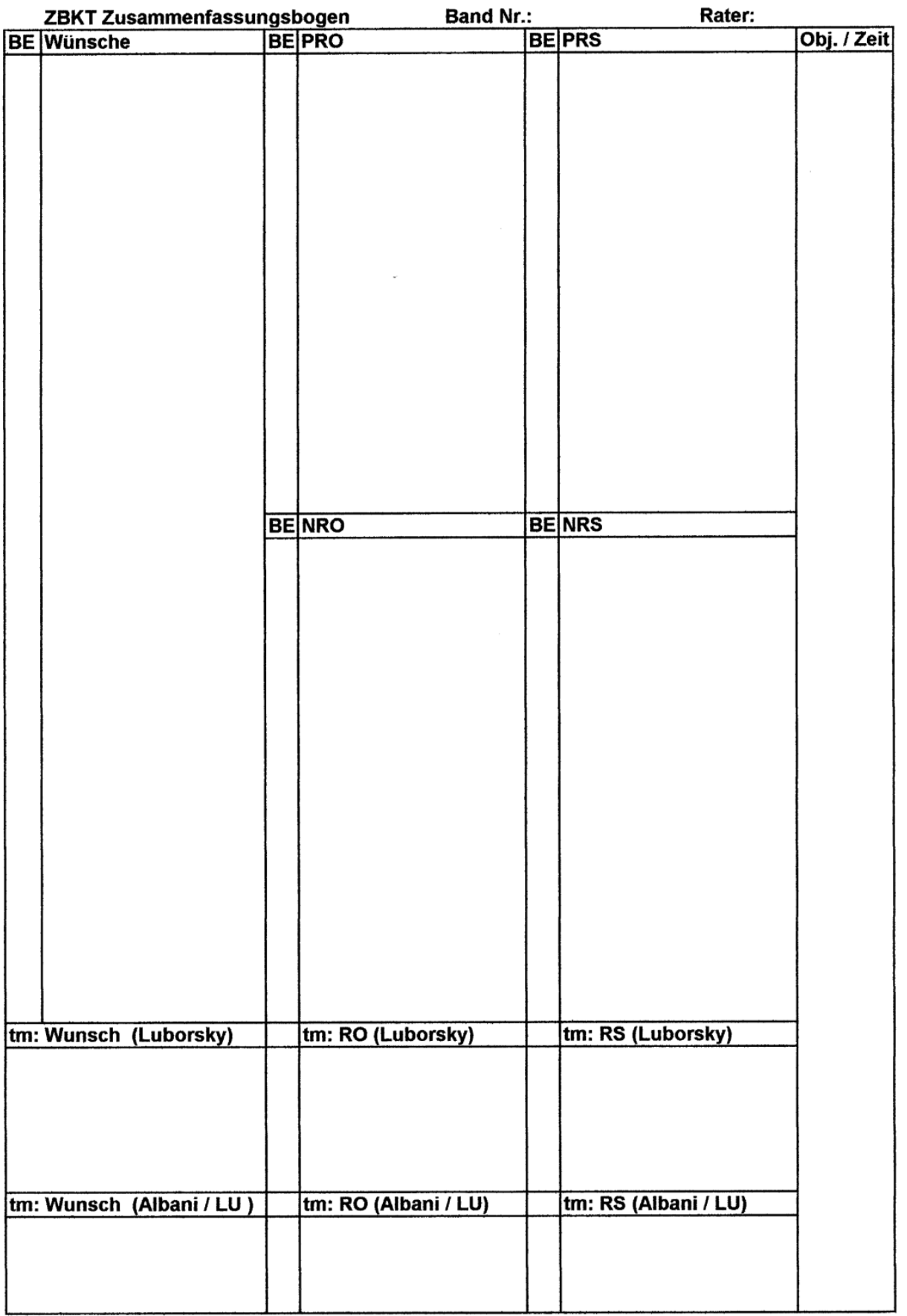




\section{Literaturverzeichnis}

- Albani C, Villmann TH, Villmann B, Körner A, Geyer M, Pokorny D, Blaser G, Kächele H (1999): Kritik der kategorialen Strukturen der Methode des zentralen Beziehungs-Konflikt-Themas (ZBKT). Psychother Psychosom Med Psychol 49, 408421

- Albani C, Pokorny D, Blaser G, Grüninger S, König S, Marschke F, Geissler I, Koerner A, Geyer M, Kächele H (2002a): Reformulation of the Core Conflictual Relationship Theme (CCRT) Categories: The CCRT-LU Category System. Psychother Res 12, 319-338

- Albani C, Pokorny D, Blaser G, König S, Geyer M, Thomä H, Kächele H (2002b): Zur empirischen Erfassung von Übertragung und Beziehungsmustern. Eine Einzelfallanalyse. Psychother Psychosom, Med Psychol 52, 226-235

- Albani C, Pokorny D, Blaser G, Kächele H: Beziehungsmuster und Beziehungskonflikte, Theorie, Klinik und Forschung, Vandenhoeck und Ruprecht, Göttingen 2008

- APA (American Psychiatric Association) Mental Health Service. Diagnostic and Statistical Manual of Mental Disorders 1952, Washington, DC. (DSM I, 1952; DSM II, 1968; DSM III, 1980; DSM IIIR 1987; DSM IV 1994; DSM IV TR (Text Revision) 2000; DSM V für 2013 geplant). Quelle: www.apa.org, Juni 2012)

- Barber JP, Crits- Christoph P, Luborsky L: A guide to the CCRT standard categories and their classification; In: Luborksy L, Crits-Christoph P (Hrsg.): Understanding transference, The CCRT-Method, Basic Books, New York 1990, 37-50

- Barber JP, Luborsky L, Crits-Cristoph P, Diguer L (1995): A Comparison of core conflictual relationship themes before psychotherapy and during early sessions. J Consult Clin Psychol 63, 145-148

- Becker ES, Margraf J: Generalisierte Angststörung: Ein Therapieprogramm. 2. Auflage, Beltz Verlag, Weinheim/Basel 2007

- Benjamin LS: Interpersonal diagnosis and treatment of personality disorders. Guilford Press, New York 1993

- $\quad$ Benkert O, Hippius H (Hrsg.): Kompendium der psychiatrischen Pharmakotherapie 7. Auflage, Springer Medizin Verlag, Heidelberg 2009, 321-333 
- Bielski RJ, Bose A, Chang CC (2005): A double-blind comparison of Escitalopram and Paroxetine in the long-term treatment of generalized anxiety disorder. Ann Clin Psychiatry, 17(2), 65-69

- Chance SE, Bakeman R, Kaslow NJ, Farber E, Burge-Callaway K (2000): Core conflictual relationship themes in patients diagnosed with borderline personality disorder who attempted, or who did not attempt suicide. Psychother Res 10(3), 337355

- Cierpka M, Zander B, Krannich S, Reich G, Ratzke K, Homburg H, Staats H, Seide I (1992): Differences in Conflictual Relationship Themes of male and female students. Presentation at the Society for Psychotherapy Research Conference, Berkeley. In: Staats H, Die Bestimmung von Beziehungsmustern und zentralen Beziehungskonfliktthemen in diagnostischen Interviews und Gruppensitzungen, Med. Habil. Schrift Göttingen 2001

- Clayton AH, Pradko JF, Croft HA, Montano CB, Leadbetter RA, Bolden-Watson C, Bass KI, Donahue RM, Jamerson BD, Metz A (2002): Prevalence of sexual dysfunction among newer antidepressants. J Clin Psychiatry 63(4), 357-366

- Crits-Christoph P, Demorest A (1988): List of Standard categories ( $2^{\text {nd }}$ Edition). Unveröffentlichtes Manuskript, zitiert nach Barber et al. 1990. Übersetzung in Luborsky et al. 1992, S. 25-27

- Crits-Christoph P, Baranackie K (1992): The quantitative assessment of relationship themes method: A merger of SASB and CCRT. Paper presented at the Society for Psychotherapy Research meeting, Berkeley. In: Staats H: Das Zentrale Thema der Stunde, Vandenhoeck und Ruprecht, Göttingen 2004, 37

- Crits-Christoph P, Luborsky L, Dahl L, Popp C, Mellon J, Mark D (1988): Clinicians can agree in assessing Relationship Patterns in Psychotherapy. The Core Conflictual Relationship Theme Method. Arch Gen Psychiatry 45, 1001-1004

- Crits-Christoph P, Crits Christoph K, Wolf-Palacio D, Fichter M, Rudick D: Brief Supportive-Expressive Psychodynamic Therapy for Generalized Anxiety Disorder. In: Barber JP, Crits-Christoph P (Hrsg.): Dynamic Therapies for Psychiatric Disorders, Basic Books, New York 1995, 44-83

- DIMDI (Deutsches Institut für medizinische Dokumentation und Information): ICD 10-GM (German Modification). Aktuell: ICD-10 GM Version 2012. Quelle: www.dimdi.de, Oktober 2012

- Di Nardo PA, Moras K, Barlow DH, Rapee RM, Brown TA (1993). Reliability of DSM-III-R Anxiety Disorder Categories - Using the Anxiety Disorders Interview Schedule - Revised (ADIS-R), Arch Gen Psychiatry, 50(4), 251-256 
- Drapeau M, Perry JC (2004): Interpersonal conflicts in borderline personality disorder: An exploratory study using the CCRT-LU. Swiss J Psychol 63(1), 53-57

- Freud S (1895): Über die Berechtigung von der Neurasthenie einen bestimmten Symptomenkomplex als Angstneurose abzutrennen. In: Gesammelte Werke aus den Jahren 1892-1899. Band 1. 6. Auflage, S. Fischer Verlag, Frankfurt am Main1992

- Freud S (1912): Zur Dynamik der Übertragung, 3. Auflage, S. Fischer Verlag, Frankfurt am Main 2006, 39-48

- Greenson RR: The Techniques and Practice of Psychoanalysis Volume I, New York 1967. Deutsch: Technik und Praxis der Psychoanalyse Band 1, vierte Auflage, Ernst Klett Verlage GmbH u. Co. KG., Stuttgart 1986

- Hamilton M (1959): Hamilton Anxiety Scale. The assessment of anxiety states by rating. Br J Med Psychol 32, 50-55

- Horowitz MJ: Converging several methods of inferring person schemas. In: Horowitz MJ (Hrsg.) Person schemas and maladaptive interpersonal patterns, Univ. Chicago Press, Chicago 1991, S. 303-310

- Hoyer J, Becker ES, Beesdo K, Wittchen HU (2003): Epidemiologie und nosologischer Status der Generalisierten Angststörung. Z Klin Psychol Psychother $\underline{32(4)}, 267-275$

- Janssen PL, Joraschky P, Tress W (Hrsg.): Leitfaden Psychosomatische Medizin und Psychotherapie, Deutscher Ärzte Verlag, Köln 2006

- $\quad$ Kessler RC, Berglund P, Demmler O, Jin R, Merikangas KR, Walters EE (2005): Lifetime prevalence and age-of-onset distributions of DSM IV disorders in the national Comorbidity Survey Replication. Arch Gen Psychiatry 62(6), 593-602

- König K (1986): Angst und Persönlichkeit - Das Konzept vom steuernden Objekt und seine Anwendungen, 6. Auflage, Vandenhoeck und Ruprecht, Göttingen 2000, 16-22

- König K: Übertragungsanalyse, Vandenhoeck und Ruprecht, Göttingen 1998, 11-12

- $\quad$ Körner A, Albani C, Villmann T, Pokorny D, Geyer M (2002): Alternative Clusterstrukturen für die ZBKT-Methode (Zentrales Beziehungskonfliktthema). Psychother Psychosom Med Psychol 52, 363-367

- Landis JR, Koch GG (1977): The measurement of observer agreement for categorical data, Biometrics $\underline{33}, 159-174$ 
- Leibing E, Winkelbach C, Leichsenring F (2003): Die generalisierte Angststörung. Darstellung eines kognitiv behavioralen Behandlungsmanuals. Verhaltensther Psychosoz Prax $\underline{35}, 517-529$

- Leichsenring F, Winkelbach C, Leibing E (2005): Psychoanalytisch-orientierte Fokaltherapie der generalisierten Angststörung: Ein Manual. Psychotherapeut 50, 258-264

- Leichsenring F, Salzer S, Jaeger U, Kächele H, Kreische R, Leweke F, Rüger U, Winkelbach C, Leibing E (2009): Short term psychodynamic psychotherapy and cognitive behavioral therapy in generalized anxiety disorder : a randomized, controlled trial. Am J Psychiatry 166, 875-881

- Lenze EJ, Mulsant BH, Shear MK, et al. (2005): Efficacy and tolerability of Citalopram in the treatment of late life anxiety disorders : results from an 8-week randomized, placebo-controlled trial. Am J Psychiatry 162(1), 146-150

- Luborsky L: Measuring a pervasive psychic structure in psychotherapy: The core conflictual relationship theme. In: Communicative structures and psychic structures, hrsg. v. Freedman N, Grand S; Plenum Press, New York 1977, 367-395

- Luborsky L (1978). The Relationship Anecdotes Paradigm (RAP) Interview: A TATlike Method using actual narratives. Unveröffentlichtes Manuskript

- Luborsky L: The early life of the idea for the core conflictual relationship theme method. In: Luborsky L, Crits-Christoph P (Hrsg.) Understanding transference - The CCRT-Method. $2^{\text {nd }}$ Edition, American Psychological Association, Washington DC 1998a, 3-13

- Luborksy L: The Convergence of Freud's observations about transference and the CCRT evidence. In: Luborsky L, Crits-Christoph P (Hrsg.): Understanding transference - The CCRT-Method. $2^{\text {nd }}$ Edition, American Psychological Association, Washington DC 1998b, 307-325

- Luborsky L: Einführung in die analytische Psychotherapie: Ein Lehrbuch. 3. Auflage, Vandenhoeck und Ruprecht, Göttingen 1999

- $\quad$ Luborsky L, Crits-Christoph P: Understanding transference - The CCRT-Method. $2^{\text {nd }}$ Edition. American Psychological Association, Washington DC 1998

- Luborsky L, Mellon J, van Ravenswaay P, Childress A, Cohen KD, Hole AV, Ming S, Crits-Christoph P, Levine FJ, Alexander K (1985): A verification of Freud`s grandest clinical hypothesis: the transference. Clin Psychol Rev $\underline{5}, 231-246$ 
- $\quad$ Luborsky L, Albani C, Eckert R (1991): Manual zur ZBKT-Methode, Übersetzung und deutsche Bearbeitung mit Ergänzungen der Ulmer ZBKT-Arbeitsgruppe.

Abteilung Psychotherapie des Universitätsklinikums Ulm (engl. Manual publiziert in Luborsky L, Crits-Christoph P, Understanding transference. The CCRT-Method. Basic Books, New York 1990)

- Luborsky L, Barber J, Diguer L (1992): The meanings of the narratives told during psychotherapy. The fruits of a new observational unit. Psychother res $\underline{2}, 277-290$

- Luborsky L, Luborsky E, Diguer L, Schmidt K, Dengler D, Faude J, Morris M, Schaffler P, Buchsbaum H, Emde R: Stability of the CCRT from age 3 to 5. In: L. Luborsky und P. Crits-Christoph (Hrsg.) Understanding transference - The CCRT Method, $2^{\text {nd }}$ Edition, American Psychological Association, Washington DC 1998, 233-251

- Luborsky L, Diguer L, Kächele H, Dahlbender R, Waldinger R, Freni S, Krause R, Frevert G, Bucci W, Drouin M, Fischmann T, Seganti A, Wischmann T, Hori S, Azzone P, Pokorny D, Staats H, Zoble H, Grenyer B, Soldz S, Anstadt T, Schauenburg H, Benninghoven D, Stigler M, Tchesnova I: A guide to the CCRT`s Method, discoveries and Future. Psychotherapie und Psychosomatische Medizin, Universitätsklinikum Ulm, Ulm 1999

- Maier W, Gansicke M, Freyberger HJ, Linz M, Heun R, Lecrubier Y (2000): Generalized anxiety disorder (ICD-10) in primary care from a cross-cultural perspective: A valid diagnostic entitiy? Acta Psychiatr Scand, 101, 29-36

- Margraf J, Schneider S (Hrsg.): Lehrbuch der Verhaltenstherapie, Band 2, 3. Auflage, Springer Medizin Verlag, Heidelberg 2009

- Movig KL, Janssen MW, de Waal MJ, Kabel PJ, Leufkens HG, Egberts AC (2003): Relationship of serotonergic antidepressants and need for blood transfusion in orthopedic surgical patients. Arch Intern Med, 163(19), 2354-2358

- Murray HA: Explorations in personality: A clinical and experimental study of fifty men of collage age. Oxford University Press, Oxford 1938

- Petrovic M, Mariman A, Warie H, Afschrift M, Pevernagie D (2003): Is there a rationale for prescription of Benzodiazepines in the elderly? Review of the literature. Acta Clin Belg, $\underline{58(1)}, 27-36$

- Pfeffer AZ (1963): The meaning of the analyst after analysis. J Am Psychoanal Assoc, 11, 229-244. In: Staats H, Das zentrale Thema der Stunde, Vandenhoeck und Ruprecht, Göttingen 2004, 37-38 
- $\quad$ Popp C, Diguer L, Luborsky L, Faude J, Johnson S, Morris M, Schaffer N, Schaffler P, Schmidt KA (1996): Repetitive relationship themes in waking narratives and dreams. J Consult Clin Psychol, 64(5), 1073-1078

- Reimer C, Eckert J, Hautzinger M, Wilke E (Hrsg.): Psychotherapie, Ein Lehrbuch für Ärzte und Psychologen, 3. Auflage, Springer Medizin Verlag, Heidelberg 2007, 481482

- Sacchi MC (2005). The CCRT LU-Method: a study of a single case. Psychological Diploma Paper, University of Milano-Biococca. In: Albani C, Pokorny D, Blaser G, Kächele H. (Hrsg.) Beziehungsmuster und Beziehungskonflikte, Theorie, Klinik und Forschung. Vandenhoeck und Ruprecht, Göttingen 2008, 122-124

- Staats H (1996): Geschlechtsunterschiede in Erzählungen von Patientinnen und Patienten zu Beginn einer Paartherapie. Gruppenpsychother Gruppendyn, 32, 366-374

- Staats H, Biskup J: Anwendung der Methode des „Zentralen Beziehungskonfliktthemas“ (ZBKT) in der Gruppentherapie. In: Methoden der Empirischen Gruppenpsychotherapieforschung - Ein Handbuch, Hrsg. V. Strauß B, Eckert J, Tschuschke V, Opladen 1996, 360-374

- Tonks A (2003): Treating generalized anxiety disorder. BMJ, 326 (7391), 700-702

- Tress, W, Henry WP, Strupp HH, Reister G, Junkert B (1990): Die strukturale Analyse Sozialen Verhaltens (SASB) in Ausbildung und Forschung. Z Psychosom Med Psychother 36, 240-257

- Uhmann S, Beesdo-Baum K, Becker E, Hoyer J (2010): Specifity of Interpersonal Problems in Generalized Anxiety Disorder Versus Other Anxiety Disorders and Depression. J Nerv Ment Dis, 198 (11), 847-851

- Vanheule VS, Demset M, Rosseel Y, Meganck Reitske (2006): Welche Kategorien des zentralen Beziehungs-Konflikt-Themas stehen in Zusammenhang mit Depressions-Syndromen? Eine explorative Studie. Z Psychosom Med Psychother, $\underline{52}, 172-189$

- Vicari A, Fabi G, Clementel C, Gottarelli L, Casonato M (2003): Predicati del sistema di categorie CCRT-LU. Psicoterapia 27, 41-43. In: Albani C, Pokorny D, Blaser G, Kächele H. (Hrsg.) Beziehungsmuster und Beziehungskonflikte, Theorie, Klinik und Forschung. Vandenhoeck und Ruprecht, Göttingen 2008, 122-124

- Weisberg RB, Dyck I, Culpepper L, Keller MB (2007). Psychiatric treatment in primary care patients with anxiety disorders: A comparison of care received from primary care providers and psychiatrists. Am J Psychiatry 164, 276-282 
- Werling VAC: Darstellung der Ziele und Probleme von Patienten mit Generalisierter Angststörung. Vergleich einer Patientengruppe in psychotherapeutischer Behandlung mit einer Gruppe von Nicht-Patienten. Med. Diss. Göttingen 2010

- Wiegand FC: Veränderungen von Problemen und Zielen im Therapieverlauf: eine Untersuchung von verhaltenstherapeutisch und psychodynamisch behandelten Patienten mit Generalisierter Angststörung. Med. Diss. Göttingen 2010

- Williams LJ, Henry MJ, Berk M, Dodd S, Jacka FN, Kotowicz MA, Nicholson GC, Pasco JA (2008): Selective serotonin reuptake inhibitor use and bone mineral density in women with a history of depression. Int Clin Psychopharmacol, 23(2), 84-87

- Wittchen HU, Pfister H: DIA-X Interview, Frankfurt: Swets Test Services, 1997

- Wittchen HU, Zhao S, Kessler RC, Eaton WW (1994): DSM-III-R generalized anxiety disorder in the national comorbidity survey. Arch Gen Psychiatry 51, 355-364

- Wittchen HU, Zaudig M, Fydrich T: SKID. Strukturiertes Klinisches Interview für DSM-IV. Achse I und II. Handanweisung. Göttingen, Hogrefe 1997

- Wittchen HU, Hoyer J, Friis R (2001): Generalized anxiety disorder - a risk factor for depression? Int J Methods Psychiatric Res 10(1), 52-57

- Yonkers KA, Dyck IR, Warshaw M, Keller MB (2000): Factors predicting the clinical course of generalized anxiety disorder. Br J Psychiatry 176, 544-549

- Zander B, Strack M, Cierpka M, Reich G, Staats H (1995). Coder agreement using the German edition of Luborsky`s CCRT method in videotaped or transcribed RAP interviews. Psychtoher Res 5, 231-236 